\author{
James Alvin Day \\ Bachelor of Science (Hon), University of Regina, 2016
}

\begin{abstract}
A thesis
presented to Ryerson University
\end{abstract}

in partial fulfillment of the

requirement for the degree of

Master of Science

in the program of

Biomedical Physics

Toronto, Ontario, Canada, 2019

CJames Day, 2019 


\section{AUTHOR'S DECLARATION FOR ELECTRONIC SUBMISSION OF A THESIS}

I hereby declare that I am the sole author of this thesis. This is a true copy of the thesis, including any required final revisions, as accepted by my examiners.

I authorize Ryerson University to lend this thesis to other institutions or individuals for the purpose of scholarly research.

I further authorize Ryerson University to reproduce this thesis by photocopying or by other means, in total or in part, at the request of other institutions or individuals for the purpose of scholarly research.

I understand that my dissertation may be made electronically available to the public.

James Day 


\title{
A SIMULATION OF A NOVEL HIGH QUANTUM EFFICIENCY SCINTILLATING FIBER DETECTOR WITH ANTI- SCATTER PROPERTIES FOR MEGAVOLTAGE X-RAY IMAGING
}

\author{
Master of Science, 2019 \\ James Day \\ Biomedical Physics \\ Ryerson University
}

\begin{abstract}
To further develop a MV x-ray portal imaging device with high detection efficiency and adequate spatial resolution for image guided radiation therapy, the experimental results for a prototype detector were matched using Monte-Carlo software to then improve upon the design. The simulation and experiment were carried out using a $6 \mathrm{MV}$ beam from a linear accelerator machine. An adequate match was obtained with the spatial resolution matching up to a MTF value of 0.2 and then diverging and the total signal registered in the central fiber was matched for field sizes ranging from $3 \mathrm{~cm}$ by $3 \mathrm{~cm}$ to $20 \mathrm{~cm}$ by $20 \mathrm{~cm}$ for $5 \mathrm{~cm}, 15 \mathrm{~cm}$ and $25 \mathrm{~cm}$ air gaps within 3\%. The design was altered from a hexagonal array of round double cladded fibers to a square array of single cladded square fibers. The spatial resolution was improved from $0.242 \mathrm{Ip} \mathrm{mm}^{-1}$ to $0.359 \mathrm{Ip} \mathrm{mm}^{-1}$ at an MTF value of 0.5 from the original design to a square array of square fibers $0.5 \mathrm{~mm}$ wide separated by $0.25 \mathrm{~mm}$ of lead foil. With further optimization of the detector design it may be possible to increase spatial resolution for $\mathrm{MV}$ x-ray imaging while maintaining an adequate detection efficiency.
\end{abstract}




\section{Acknowledgements}

I would like to acknowledge my supervisor, Dr. Geordi Pang for his support towards my research. His expertise in research topic always helped me when I was stuck. Although I did not end up completing the requirements for CAMPEP accreditation during my master's degree in Medical Physics I had the support to pursue the majority of required courses and a future PHD at Ryerson to complete the CAMPEP program.

I would like to also thank my co-supervisor, Dr. Yuan Xu, his knowledge in imaging and programming libraries was always helpful. His courses helped me to understand the goals of a good imaging device. Even though I did not take his Advanced Imaging course, the textbook that he recommended for that course was useful for understanding the finer details in my research.

I would like to specially thank Dr. Aram Teymurazyan for helping me secure a spot in the master's program at Ryerson through both his help with finding a potential supervisor and his mentorship during the years of 2016 and 2017. The skills I learned from being a lab assistant under him proved to be invaluable. His continued input into my research progress throughout my master's degree always served to keep me on the right track and his advice was always fruitful.

I would like to also thank my committee member Dr. James Grafe for not only his useful comments during the committee meetings but also the wealth of knowledge I gained from the courses I took from him. The knowledge I gained during his introduction to radiation physics and radiation therapy courses was used extensively to aid in my research and understanding of the topic of external radiation therapy. I would like to express my appreciation towards the technical staff at Ryerson. Kevin Liu, Matthew Forest and Graham Pearson were always helpful whether it was for equipment advice or a second look at technical problems that I could not solve.

I would like to acknowledge the Natural Sciences and Engineering Research Council of Canada (NSERC) for funding this project.

Lastly, I would like to thank my family for their continued support during my masters. 


\section{Contents}

Author's Declaration for Electronic Submission of a Thesis ................................................................ ii

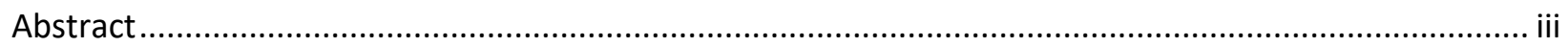

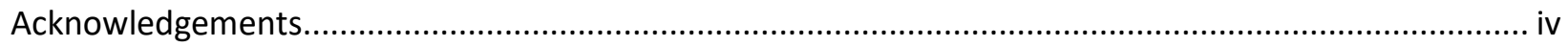

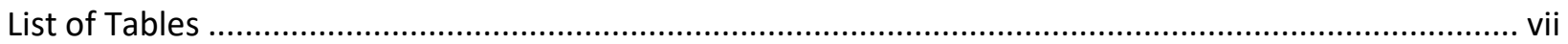

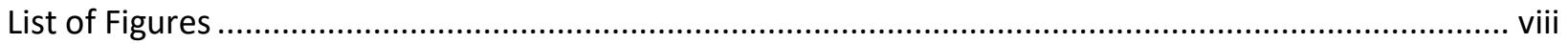

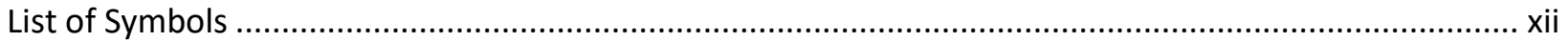

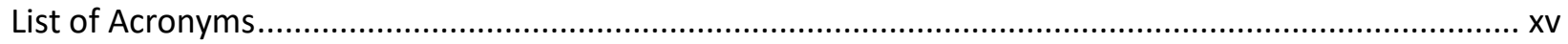

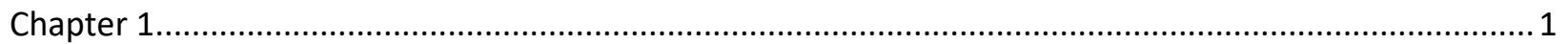

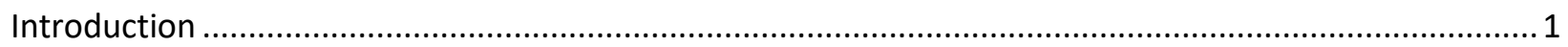

1.1 The Importance of Imaging in Radiation Therapy ............................................................................. 2

1.2 The Physics and Image Quality Quantities for Imaging Devices ...................................................... 3

1.2.1 The Principle Physical Interactions of Particles with Matter for Imaging .................................... 3

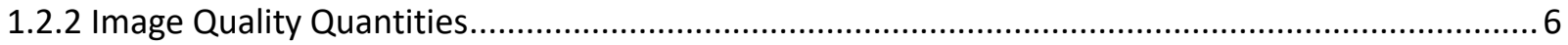

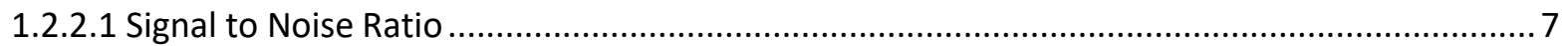

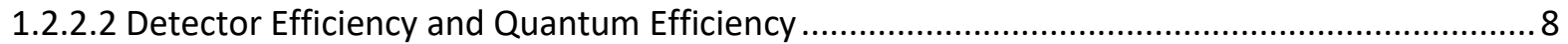

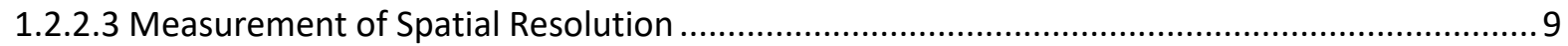

1.2.2.4 The Discrete Modulation Transfer Function, the Discrete Fourier Transform and the Fast

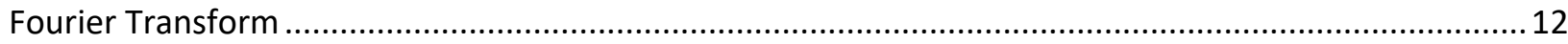

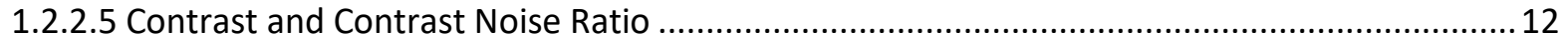

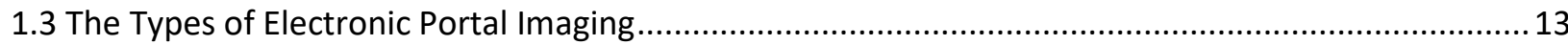

1.4 Early Improvements to Electronic Portal Imaging ......................................................................... 14

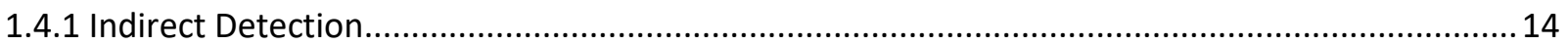

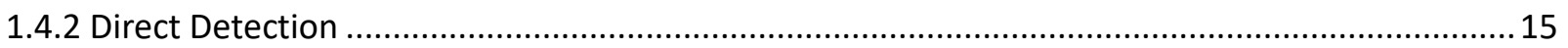

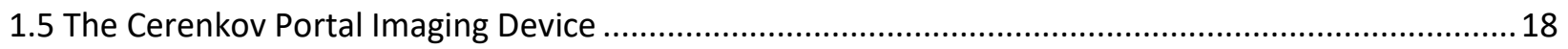

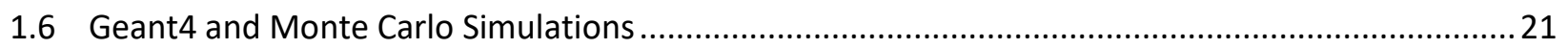

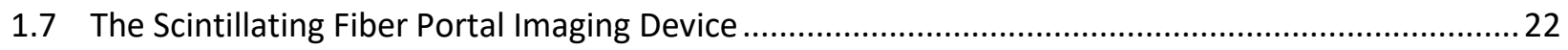

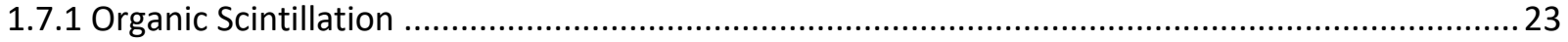

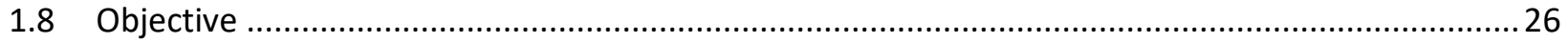

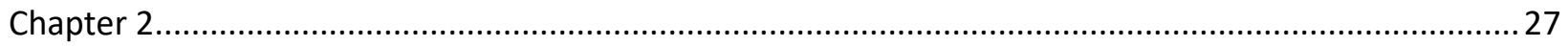

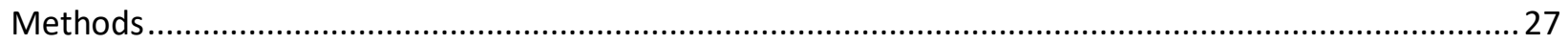

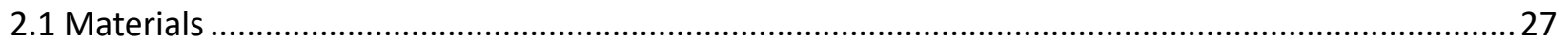

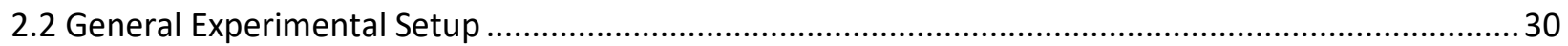




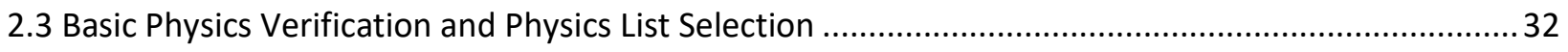

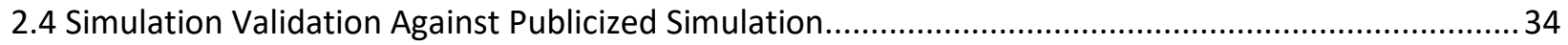

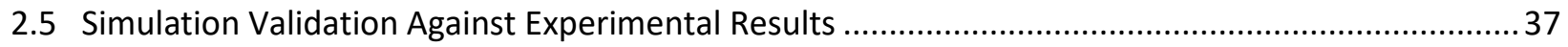

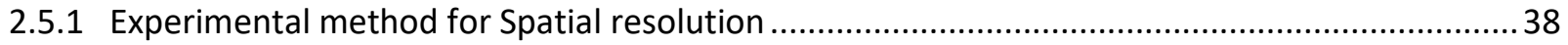

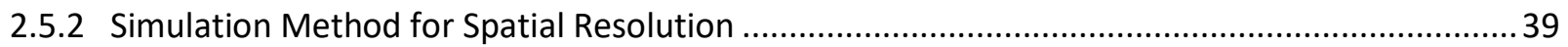

2.5.3 Experimental Method for Scatter Primary Ratio ..................................................................... 40

2.5.4 Simulation Method for Scatter Primary Ratio ....................................................................4

2.5.4.1 Determining the Equivalent Field Size ........................................................................ 46

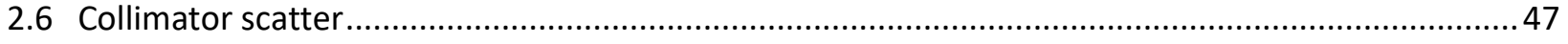

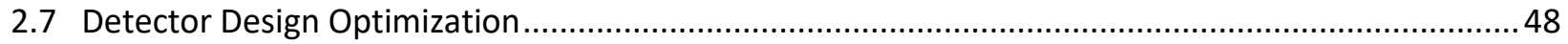

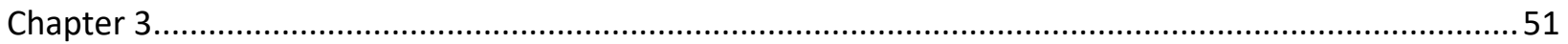

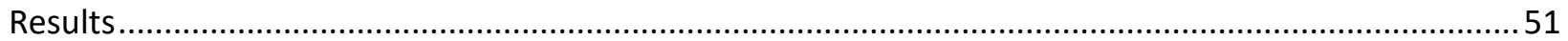

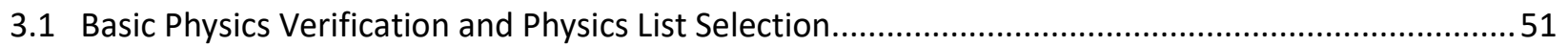

3.2 Simulation Validation Against Publicized Simulation .......................................................................53

3.3 Simulation Validation Against Experimental Results ...................................................................59

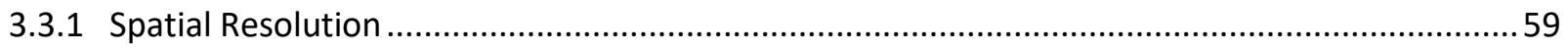

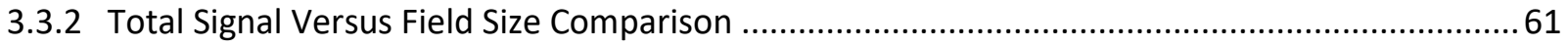

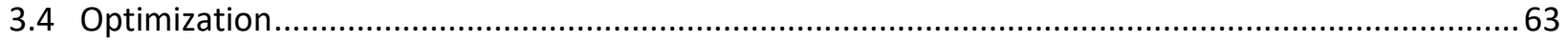

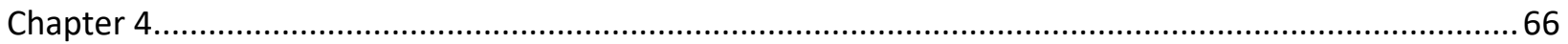

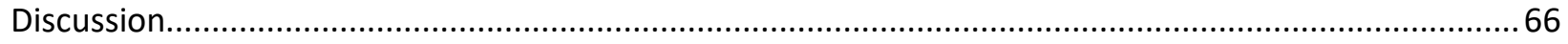

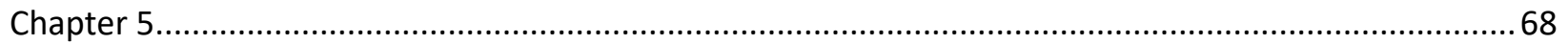

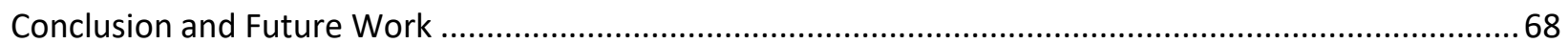

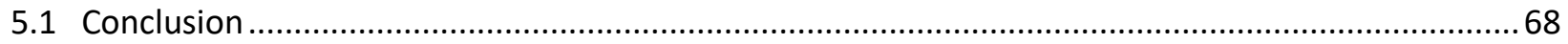

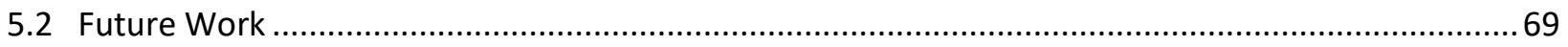

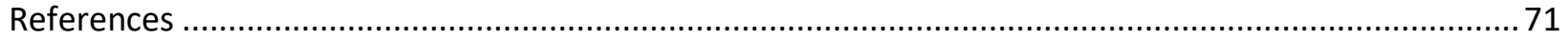




\section{List of Tables}

1.1 Properties of the main attenuation interactions that result in photon energy absorption. Rayleigh scattering was omitted since it does not absorb any energy from the photon. The table was adapted from the original source $[11] \ldots \ldots \ldots \ldots \ldots$

1.2 NR values for various doses at therapeutic energies compared to the SNR from a

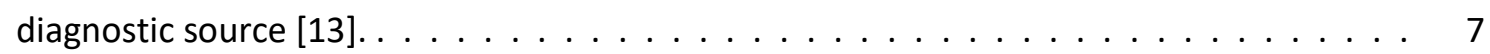

2.1 The materials used to make the fibers used for the prototype detector. The chemical formulas for the core and the inner cladding are well known but the formula for the outer cladding was a mystery. As a result, tetrafluoroethylene was used in the simulation. The material was forced to have the same density and refractive index as the outer cladding

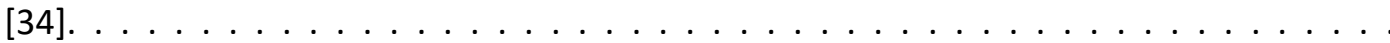




\section{List of Figures}

1.1 Clinical LINAC used to gather the experimental data for this project. . . . . . . . . . . . . 1

1.2 Here $\tau$ is the photoelectric (PE) cross section, $\sigma$ is the Compton cross section and $\mathrm{k}$ is the pair

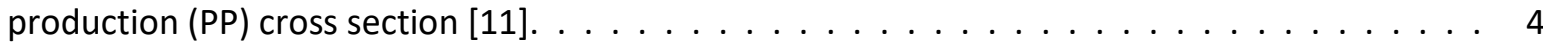

1.3 The different types of charged particle interactions $[11] \ldots \ldots \ldots \ldots$

1.4 An example of how spatial resolution is ultimately calculated. Spatial resolution depends on how close two object can be from each other and still be distinguishable from one another $[16] \ldots \ldots \ldots \ldots \ldots \ldots \ldots \ldots \ldots$

1.5 An example of the contrast differences between MTF values. The lines become less defined as the MTF value is reduced $[16] \ldots \ldots \ldots \ldots \ldots \ldots$

1.6 An illustration of two types of X-ray detection method for portal imaging. (a) shows the indirect method which uses a converting phosphor and photodiode to generate and signal. (b) shows the direct detection method where the generated electrons are collected. A photoconductor with a collection electrode are used to generate a signal $[14] \ldots \ldots \ldots \ldots \ldots$

1.7 An early idea for a high quantum efficiency indirect detection type portal imager. The elements are aligned with the $x$-ray source for an unknown field size $[19] \ldots \ldots \ldots$

1.8 An early design of a direct detection portal imager. Top (a) shows the composition of a single element and (b) shows multiple elements, they are arranged in a trapezoidal configuration like the previous design. Bottom shows a full scale detector $[22] \ldots \ldots \ldots \ldots$

1.9 The spatial resolution measured via MTF for the detector shown in figure 1.8. The thinnest detector appears to consistently have the higher spatial resolution [22] . . . . . . . . . . .

1.10 An indirect portal imager that relies on Cerenkov radiation to convert the incoming $\mathrm{x}$-rays into optical photons via the generated super luminal (relative to the medium) electrons. The absorber is an opaque black resin with similar properties to water. The fibers are again slanted

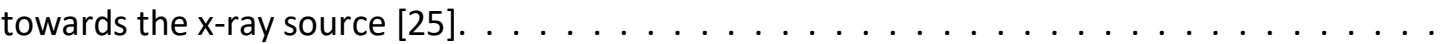

1.11 The measured spatial resolution for the detector describe in figure 1.10. The thickness of this detector does not appear to significantly affect the spatial resolution over the range of spatial frequencies shown $[25] \ldots \ldots \ldots \ldots \ldots \ldots \ldots \ldots \ldots$ 
1.12 This figure shows the 'energy levels of an organic molecule with [a] $\pi$-electron structure'. In most cases the amount of energy to excite the molecule is greater than the amount of energy released as radiation when it relaxes $[15] \ldots \ldots \ldots \ldots \ldots \ldots$

2.1 A close-up of the construction of the prototype detector. The Fibers use were plastic scintillating fibers (Kuraray SCSF-78MJ, Kuraray Co., Ltd., Tokyo, Japan) and the epoxy was BC600 optical epoxy (Saint-Gobain Crystals, OH, USA). The components of the fibers are shown in table $I$ and the geometry of the fibers is shown in figure $2.2[34] \ldots \ldots \ldots \ldots$

2.2 The geometry of the double cladded plastic scintillating fibers (Kuraray SCSF-78MJ, Kuraray Co., Ltd., Tokyo, Japan). . . . . . . . . . . . . . . . . . . . . . . . . . . . .

2.3 General Experimental setup. The signal was measured using two scintillating fibers. One embedded in the center of the detector and one to subtract additional signal generated in the length of the fiber protruding from the detector $[34] \ldots \ldots \ldots \ldots \ldots$

2.4 An illustration of the method of to obtain the signal in the embedded portion of the scintillating fiber. The example displays the method for optical fibers however the method is

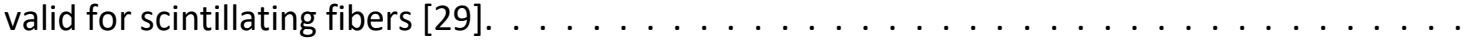
32

2.5 The polyenergetic spectrum used for the simulation validation [33] . . . . . . . . . . . . 36

2.6 The polyenergetic spectrum used in the simulation to compare to the experimental results for the prototype detector. This spectrum is from the Pinnacle 3 treatment planning system [34]. .

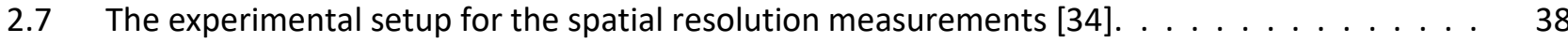

2.8 The experimentally measured line spread functions for both the $x$-axis and the $y$-axis [34] . . . 39

2.9 The Experimental setup for the scatter primary ratio versus field size measurements [34] . . . 41

2.10 The experimental results for the scatter primary ratio measurements [34] . . . . . . . . . . . 42

2.11 The method to determine the primary signal for the experimental measurements. The primary signal for each field size was determined by an extrapolation to zero field size [34]. The extrapolation function was unknown during the simulations. . . . . . . . . . . .

2.12 An example of how the total signal for various field sizes were determined. The signal for each of the pixels inside the areas were added up for each of the field sizes. The field sizes shown are the equivalent field sizes for a cone beam at the iso-center. . . . . . . . . . . .

2.13 The illustrated comparison between the effective simulation beam and the actual beam for the experiment. The blue area is the area missed by the cone beam but included in the simulation beam, the red area shows the area for the opposite of this. The differences in volume is

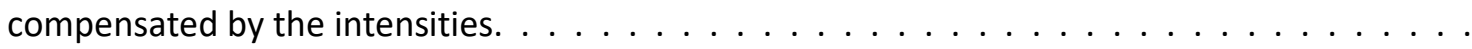


2.14 The collimator scatter factor curve for the machine used in the experiment. A semi log graph was used to clearly show the scatter factors for the smaller field sizes this is necessary since the smallest field size used in the simulation was approximately $3 \mathrm{~mm}^{2} \ldots \ldots \ldots \ldots$

2.15 Close up of the new detector design for the optimization experiments. The glue was placed in the corner of the pixels since it was thought to be easier to build the detector this way. . . . .

3.1 Prebuilt simulation code performance for attenuation. The simulation was performed using

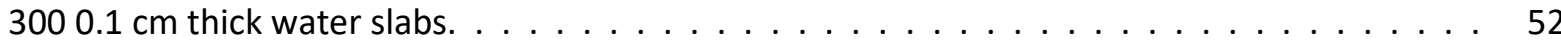

3.2 The performance for CSDA range and by extension stopping power was performed by firing electrons into an effectively infinite volume of water. . . . . . . . . . . . . . . .

3.3 The comparison between the two simulations were done with an airgap of 0 and monoenergetic $x$-ray sources. The error bars in Jaffrays data are from the size of the data points and the borders in the original plot. . . . . . . . . . . . . . . . . . . . . 54

3.4 The percentage differences for the scatter fraction measurements shown in figure 3.3. . . . . 55

3.5 X-ray fluence measurements. Primary fluence was measured by the amount of energy deposited in the phantom. If there was no energy deposited in the phantom, then the x-ray interaction with scoring area was primary. The phantom thickness was $17 \mathrm{~cm}$ and the airgap

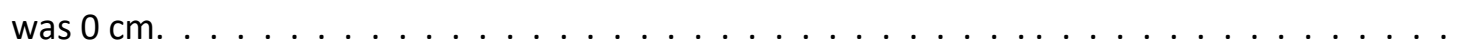

3.6 A comparison between the energy spectra of a monoenergetic beam of $x$-rays after interaction with $17 \mathrm{~cm}$ thick phantom. The energy was measured after they passed the scoring volume at an air gap of $0 \mathrm{~cm}$. This is an overlay of this simulations plot and Jaffray's plot. Both the $x$-axis and the highest point in the peaks were matched. . . . . . . . . . . . . . . . .

3.7 The rest of the comparisons made with Jaffrays simulations. Unless listed on the X-Axis as varying the airgap was $30 \mathrm{~cm}$, the phantom thickness was $17 \mathrm{~cm}$ and the field size was $30 \mathrm{~cm}$ by $30 \mathrm{~cm}$. A 6MV poly energetic spectrum was used in for all the measurements. The spectrum can be seen in figure $2.5 \ldots \ldots \ldots \ldots \ldots \ldots \ldots \ldots$

3.8 A comparison between the simulations and experiments line spread functions along the $x$ axis. The $6 \mathrm{MV}$ polyenergetic spectrum seen in figure 2.6 was used for all simulations of the detector

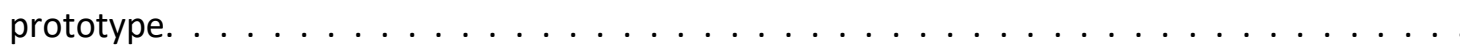

3.9 The comparison between the modulation transfer functions (MTFs) for experiment and simulation. Both MTF were generated using the same fast fourier transform code for

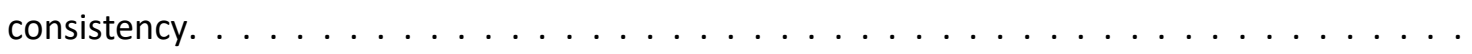


3.10 A $30 \mathrm{~cm}$ thick phantom of solid water was used centered at a distance of $100 \mathrm{~cm}$ from the

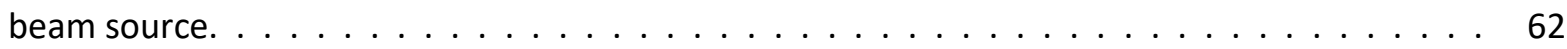

3.11 A comparison of spatial resolutions for a range of square fiber thicknesses. . . . . . . . . . . . 64

3.12 A comparison of spatial resolutions for a range of lead foils thickness for the most optimum

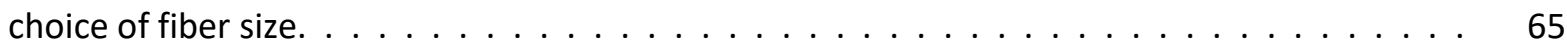




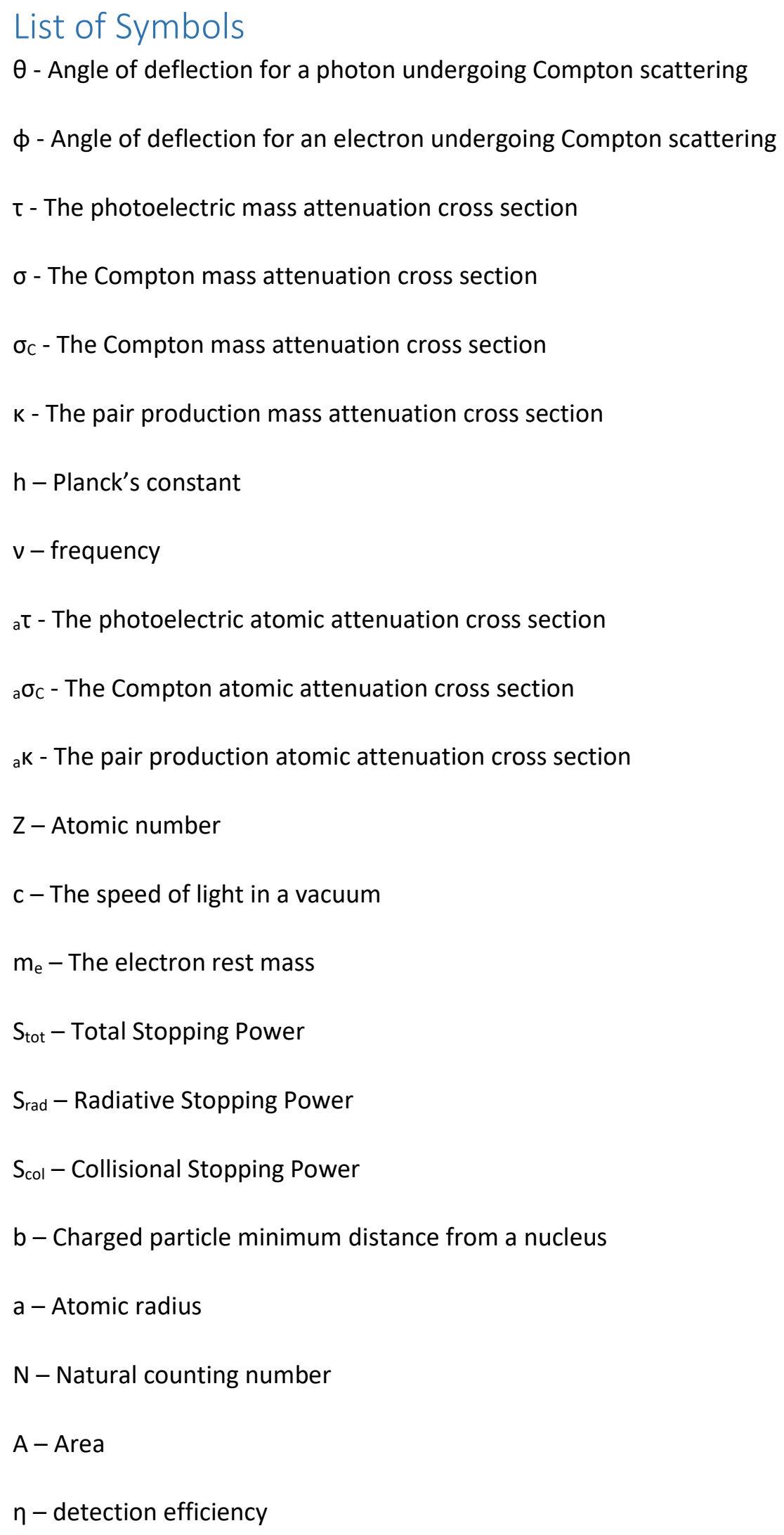


$L$ - Thickness

$\mu$ - Linear attenuation coefficient

$\phi$ - Fluence

T - Phantom transmission factor

$\bar{d}-$ Mean detector signal

$\bar{q}$ - Mean incident x-ray fluence

$f-$ Spatial frequency

$L(x)$ - Line spread function in the $x$ axis

$P(x, y)$ - Point spread function

$\mathrm{k}$ - Discrete spatial frequency

C-Contrast

S- Signal

$\sigma_{N}-$ Standard deviation of signal noise

$\mathrm{n}$ - Index of refraction

$\beta$ - Relative speed to the speed of light in a medium

kB - Birks adjustment parameter

E-Energy

$\mathrm{L}$ - Path length

S-Stopping power

F- Fluorine

C-Carbon

T - Thickness

NA - Numerical aperture 
K - Fano-factor

Range $_{C D D A}-$ Continuous slowing down approximation range

T - Total signal

S - Scatter signal

P - Primary signal 


\title{
List of Acronyms
}

\author{
LINAC - Linear Accelerator \\ MV - Megavoltage \\ CT - Computed Tomography \\ IGRT - Image Guided Radiation Therapy \\ MRI - Magnetic Resonance Imaging \\ KV - Kilovoltage \\ CBCT - Cone Beam Computed Tomography \\ PE - Photoelectric \\ PP - Pair Production \\ SNR - Signal to Noise Ratio \\ SF - Scatter Fraction \\ DQE - Detective Quantum Efficiency \\ NPS - Noise Power Spectrum \\ PSF - Point Spread Function \\ FWHM - Full Width at Half Maximum \\ DFT - Discrete Fourier Transform \\ FFT - Fast Fourier Transform \\ CNR - Contrast to Noise Ratio \\ EPID - Electron Portal Imaging Device \\ AMFPI - Active Matrix Flat Panel Imager \\ QE - Quantum Efficiency
}


CPID - Cerenkov Portal Imaging Detector

PMMA - Polymethylmethacrylate

PMT - Photomultiplier Tube

EM - Electromagnetic

NIST - National Institute of Standards and Technology

XCOM - The NIST Photon Cross Section Database

CSDA - Continuous Slowing Down Approximation

E-STAR - The NIST Stopping-power and Range Tables for Electrons

Geant4 - Geometry and Tracking 4

EGS4 - Electron Gamma Shower 4

GCUT - Gamma Cut

ECUT - Electron Cut

TPS - Treatment Planning System 


\section{Chapter 1}

\section{Introduction}

In Canada nearly half of the population is expected to be diagnosed cancer in their lifetimes with a total of $1 / 4$ of Canadian mortality as a result of this disease. The mortality rate from cancer has been in decline since 1988 from advances in screening, prevention, early detection and treatment showing declines in death rates of $32 \%$ for males and $17 \%$ for females from 1988 to 2017 . Cancer diagnosis rates are expected to rise by as much as $80 \%$ by 2030 compared to those reported in 2005 [1]. In most cases the resulting tumor is treated by either surgery, chemotherapy, radiation therapy or a combination of these treatment modalities. The most common treatment is external beam radiation therapy. External beam therapy is most commonly delivered using a linear accelerator (LINAC) seen in figure 1.1 [2]. External beam radiation therapy is useful for treating tumors in regions where surgery carries a high risk or in regions where the tumor is inoperable. The goal of radiation therapy is to kill the cancerous cells while sparing the healthy cells. The LINAC uses megavoltage (MV) $\mathrm{x}$-rays to primarily damage cancer cells by taking advantage of their high radio-sensitivity compared to healthy cells [3].

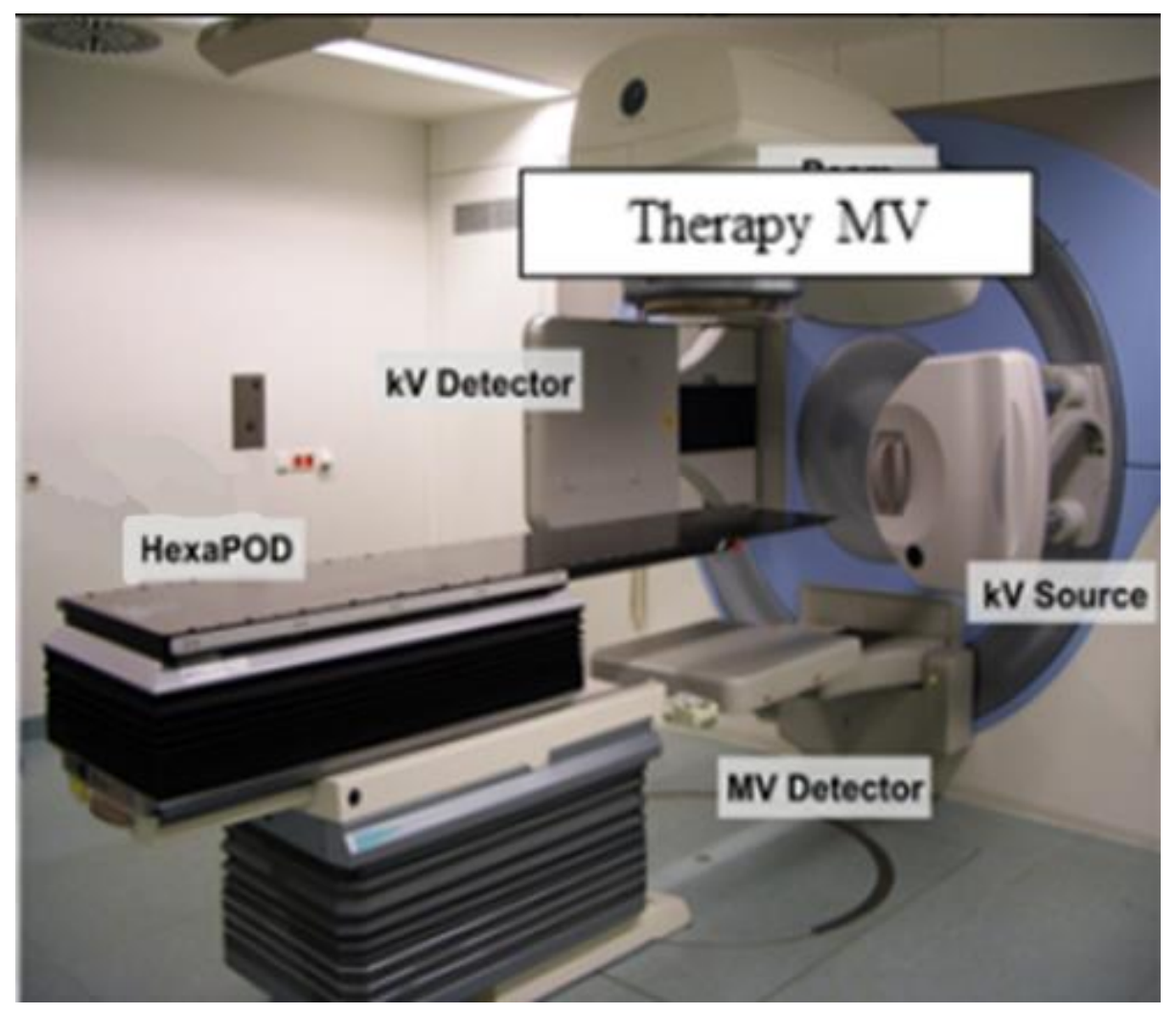

Figure 1.1 Clinical LINAC used to gather the experimental data for this project. 


\subsection{The Importance of Imaging in Radiation Therapy}

The development of radiation therapy techniques has been closely linked to medical imaging. Imaging is used for diagnosis, management and the prognosis of cancers to detect the stage, size, and location of the tumor for treatment planning. Imaging such as computed tomography (CT) with the use of treatment planning software and delivery systems have made it possible to have conformal doses delivered to the planning volume minimizing the dose received by healthy tissue [4]. The planning images are used in conjunction with skin marks and laser alignment to position the patient according to the treatment plan $[2,4]$. Imaging, performed using 2D portal imaging or 3D cone beam CT, is used to verify the accuracy of the treatment setup and to make any necessary corrections for systematic and random errors [4].

Image guided radiation therapy (IGRT) operates off the hypothesis 'Increasing the precision and accuracy of radiation delivery will reduce toxicity with potential for dose escalation and improved tumor control' [4]. Accomplishing high precision and accuracy requires strict control over geometric uncertainties to deliver a highly localized dose to the treatment volume. In addition, this requires highly accurate verification imaging and positioning of the patient for treatment with minimal setup-errors [4].

Setup errors are corrected using either offline imaging or online imaging. Offline imaging is done by imaging the patient during the therapy, allowing the first few fractions of the treatment to go uncorrected ( 2 to 5 fractions), and then reviewing the images for any systematic errors which are corrected for the following fractions. The advantage of this is an increase in throughput since the images are not reviewed during the treatment. However, the disadvantages to doing offline imaging is that random errors cannot be corrected, only the systematic errors are accounted for. Online imaging is performed at the beginning of the treatment after the initial setup is performed which increases the length of the treatment. However, this method is capable of correcting both the systematic and random errors making it the primary method of error correction in external beam radiation therapy [4].

Positioning of the patient is done initially by using markers and patient specific immobilizing masks to align the skeletal structure to within $3 \mathrm{~mm}$ accuracy $[5,6]$. However, since the organs move and change locations randomly the marked position and actual target position can have a discrepancy of up to $1 \mathrm{~cm}$ [5]. Corrections can be done using imaging techniques such as magnetic resonance imaging (MRI) or kilovoltage (KV) cone beam computed tomography (CBCT) to correct the setup errors to within 1 to $2 \mathrm{~mm}$ 
by providing high contrast anatomical images giving accurate information on the tumors' location $[7,5,6]$. CBCT can be used for both imaging of the tumor and for calculating the cumulative dose distribution through the use of correction factors to convert the attenuation coefficients [6]. Currently some verification imaging is done through MV portal imaging which has advantages of utilizing the LINAC beam such as co-registration of imaging and treatment beams and a decrease in the artifacts caused by high Z materials such as implants seen in KV imaging $[7,6,8]$. Currently setup corrections are done by comparing the bony anatomy to the reference images; because of the poor contrast from MV imaging the tumor itself is not usually visible. Megavoltage cone beam computed tomography (MV CBCT) is required to visualize the gross tumor volume (GTV). The clinical requirement for imaging is a fast, low dose system which currently limits the feasibility of using MV CBCT in practise. Currently, MV CBCT is estimated to require 10 to $15 \mathrm{MU}$ of exposure and 7 to $10 \mathrm{cGy}$ of absorbed dose for each acquisition [9]. Reducing the dose to values associated with $\mathrm{KV}$ imaging would require an imager with improved detection efficiency for MV x-rays [10].

\subsection{The Physics and Image Quality Quantities for Imaging Devices}

X-ray imaging is performed by measuring the intensity of radiation that is transmitted through an object as a function of position. The $\mathrm{x}$-rays used in both $\mathrm{KV}$ and $\mathrm{MV}$ imaging are generated by energetic electrons colliding with a material through the bremsstrahlung process. Bremsstrahlung radiation occurs when an electron travels close to the nucleus of an atom and is deflected by the electromagnetic force releasing any lost kinetic energy in the form of an $\mathrm{x}$-ray photon. The energy of the resulting $\mathrm{x}$-ray can range up to the nominal accelerator potential of the LINAC $[11,12]$. For a LINAC used in radiotherapy the nominal accelerator potential can range up to $25 \mathrm{MV}$ [11]; however, for MV CBCT an energy at or below $6 \mathrm{MV}$ is used $[6,9]$.

\subsubsection{The Principle Physical Interactions of Particles with Matter for Imaging}

For x-ray imaging, image contrast is created through three different interaction processes: the photo-electric effect, Compton scattering and pair production. The photoelectric effect is the process where a photon is absorbed by a bound electron transferring all of its energy to the electron potentially ejecting it from the atom. Compton scattering, like the photo electric effect, takes place between an 
incident x-ray and an electron in an atom; however, in Compton scattering the photon is partially absorbed by the electron. This partial absorption causes the photon to be deflected at an angle $\theta$ while the electron is deflected at another angle $\phi$ according to the conservation of energy and momentum. The final major interaction, pair production occurs above the threshold of $1.022 \mathrm{MeV}$ and by extension triplet production which occurs at a threshold of $2.044 \mathrm{MeV}$ occurs when the $\mathrm{x}$-ray photon interacts with an atom and converts into an electron positron pair. The probabilities of these interactions, shown in figure 1.2 , depend on both the energy of the $x$-ray photon and the composing material. The average energy of a $6 \mathrm{MV}$ LINAC is around $2 \mathrm{MeV}$ making the dominant interaction the Compton effect. From table 1.1, unlike for pair production and the photoelectric effect, the attenuation coefficient for Compton does not depend on the atomic number of the material; instead, the attenuation depends on the electron density [11].

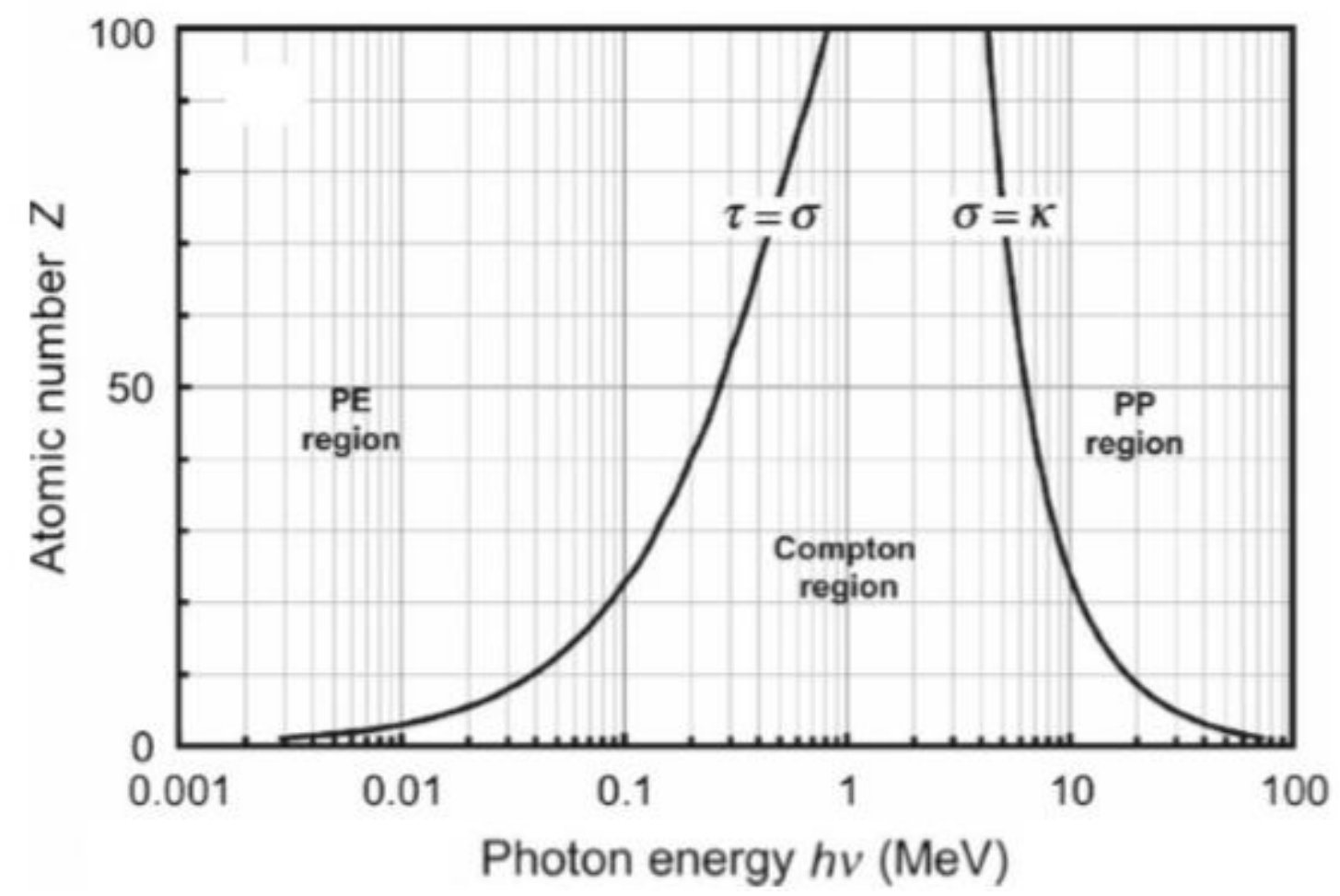

Figure 1.2 Here $\tau$ is the photoelectric (PE) cross section, $\sigma$ is the Compton cross section and $\mathrm{k}$ is the pair production (PP) cross section [11]. 


\begin{tabular}{|c|c|c|c|c|}
\hline & & & & \\
\hline $\begin{array}{l}\text { Table } 1.1 \\
\text { Pronerties of the main attenuation }\end{array}$ & & $\begin{array}{l}\text { Photoelectric } \\
\text { effect }\end{array}$ & $\begin{array}{l}\text { Compton } \\
\text { effect }\end{array}$ & $\begin{array}{l}\text { Pair } \\
\text { production }\end{array}$ \\
\hline interactions that result in photon & $\begin{array}{l}\text { Photon } \\
\text { interaction }\end{array}$ & $\begin{array}{l}\text { With whole } \\
\text { atom (bound } \\
\text { electron) }\end{array}$ & $\begin{array}{l}\text { With free } \\
\text { electron }\end{array}$ & $\begin{array}{l}\text { With nuclear } \\
\text { Coulomb field }\end{array}$ \\
\hline $\begin{array}{l}\text { energy absorption. Rayleigh } \\
\text { scattering was omitted since it does }\end{array}$ & $\begin{array}{l}\text { Mode of photon } \\
\text { interaction }\end{array}$ & $\begin{array}{l}\text { Photon } \\
\text { disappears }\end{array}$ & $\begin{array}{l}\text { Photon } \\
\text { scattered }\end{array}$ & $\begin{array}{l}\text { Photon } \\
\text { disappears }\end{array}$ \\
\hline not absorb any energy from the & $\begin{array}{l}\text { Energy } \\
\text { dependence }\end{array}$ & $\frac{1}{(h \nu)^{3}}$ & $\begin{array}{l}\text { Decreases } \\
\text { with energy }\end{array}$ & $\begin{array}{l}\text { Increases with } \\
\text { energy }\end{array}$ \\
\hline $\begin{array}{l}\text { photon. The table was adapted from } \\
\text { the original source [11]. }\end{array}$ & $\begin{array}{l}\text { Threshold } \\
\text { energy }\end{array}$ & $\begin{array}{l}\text { Shell binding } \\
\text { energy }\end{array}$ & $\begin{array}{l}\text { Shell } \\
\text { binding } \\
\text { energy }\end{array}$ & $\sim 2 m_{e} c^{2}$ \\
\hline & $\begin{array}{l}\text { Linear } \\
\text { attenuation } \\
\text { coefficient }\end{array}$ & $\tau$ & $\sigma_{\mathrm{C}}$ & $\kappa$ \\
\hline & $\begin{array}{l}\text { Atomic coef. } \\
\text { dependence } \\
\text { on } Z\end{array}$ & $\mathrm{a}^{\tau} \propto Z^{4}$ & $\mathrm{a} \sigma_{\mathrm{C}} \propto Z$ & ${ }_{a} \kappa \propto Z^{2}$ \\
\hline & $\begin{array}{l}\text { Mass coefficient } \\
\text { dependence } \\
\text { on } Z\end{array}$ & $\frac{\tau}{\rho} \propto Z^{3}$ & $\begin{array}{l}\text { Independent } \\
\text { of } Z\end{array}$ & $\frac{\kappa}{\rho} \propto Z$ \\
\hline & $\begin{array}{l}\text { Particles } \\
\text { released in } \\
\text { absorber }\end{array}$ & Photoelectron & $\begin{array}{l}\text { Compton } \\
\text { (recoil) } \\
\text { electron }\end{array}$ & $\begin{array}{l}\text { Electron- } \\
\text { positron pair }\end{array}$ \\
\hline & $\begin{array}{l}\text { Predominant } \\
\text { energy region } \\
\text { for water }\end{array}$ & $<20 \mathrm{keV}$ & $\begin{array}{l}20 \mathrm{keV}- \\
20 \mathrm{MeV}\end{array}$ & $>20 \mathrm{MeV}$ \\
\hline & $\begin{array}{l}\text { Predominant } \\
\text { energy region } \\
\text { for lead }\end{array}$ & $<500 \mathrm{keV}$ & $\begin{array}{l}500 \mathrm{keV}- \\
5 \mathrm{MeV}\end{array}$ & $>5 \mathrm{MeV}$ \\
\hline
\end{tabular}

From the interactions listed above the main type of secondary radiation is a charged particle such as an electron or a positron. The primary types of interactions that a charged particle makes with matter are: bremsstrahlung production (radiation collisions), hard collisions and soft collisions. Figure 1.3 shows the types of interactions, where soft collisions occur the furthest from the nucleus and radiative collisions occur the closest to the nucleus. Radiative interactions occur mainly with the nucleus of the atom and primarily result in a change of direction of the electron with some energy being emitted as a photon to satisfy conservation laws. Occasionally these interactions result in a large energy loss through an inelastic scattering. Hard collisions occur when the charge particle interacts with an orbital electron in the atom. This interaction results in a significant amount of energy being transferred. This results in delta radiation 
or orbital electrons being knocked free from the orbitals. Soft collisions are the most common type of interaction and are due to a distant interaction with the orbital electrons. They can result in ionization, excitation or polarization. Only a small portion of the kinetic energy is transferred per interaction, but it is frequent enough that about $50 \%$ of the energy lost is due to soft collisions. The result of these interactions is the eventual stopping of the electrons. The ability for a material to slow down and stop a given electron is known as the stopping power. This consists of radiative stopping power $\mathrm{S}_{\mathrm{rad}}$, from nuclear interactions like Bremsstrahlung and collisional stopping power $S_{\mathrm{col}}$, from the electronic interactions described by hard and soft collisions. The total stopping power of a material $\mathrm{S}_{\text {tot }}$, is given as the sum of $\mathrm{S}_{\text {col }}$ and $\mathrm{S}_{\mathrm{rad}}$ as shown in equation 1.1 [11].

$$
S_{\text {tot }}=S_{\text {rad }}+S_{\text {col }}
$$

Equation 1.1

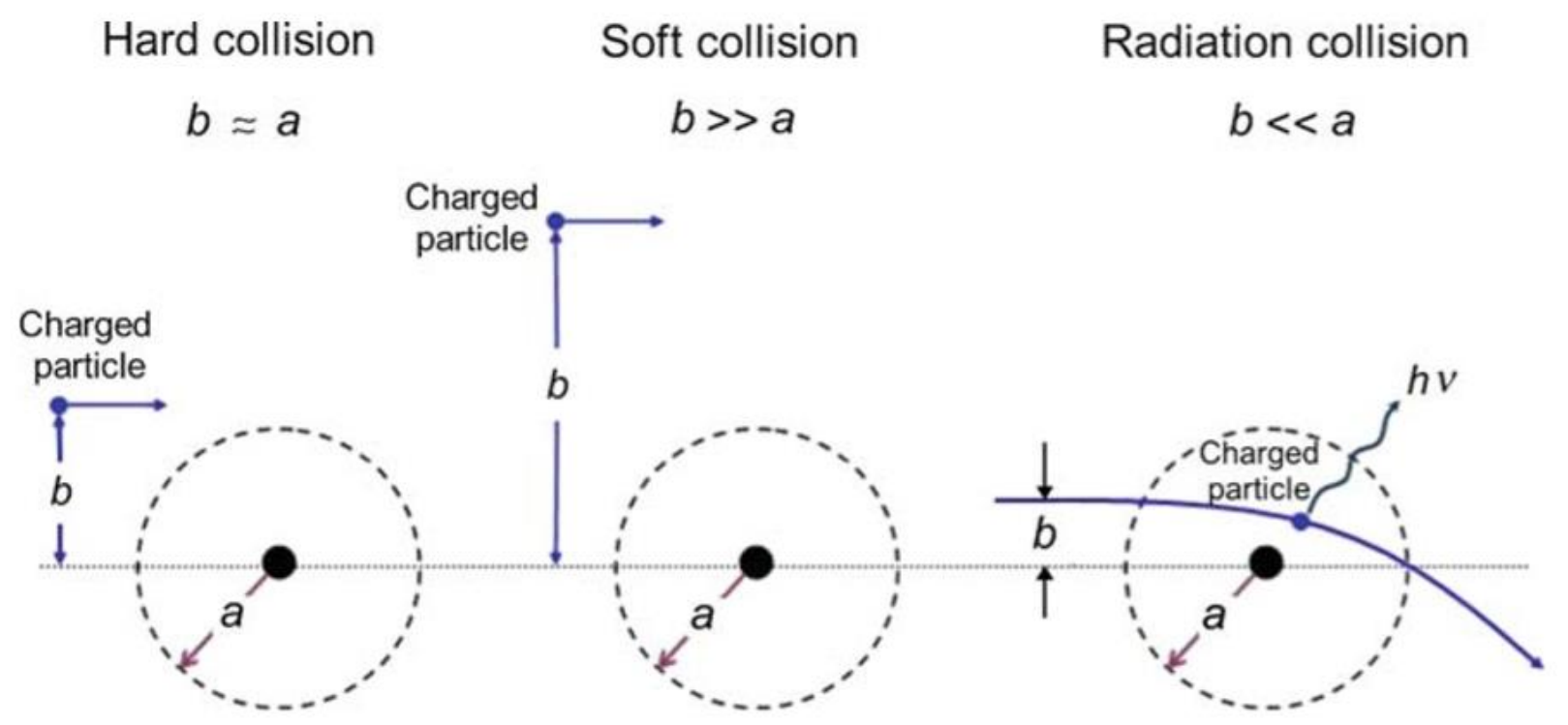

Figure 1.2 The different types of charged particle interactions [11].

\subsubsection{Image Quality Quantities}

For any biomedical imaging device there are ways that the detectors characteristics can be evaluated and compared. The most important criteria are contrast to noise ratio, signal to noise ratio and spatial resolution $[12,13]$. Additional measurements of importance to these criteria are detective 
quantum efficiency, quantum efficiency and scatter fraction which are useful measurements related to detector performance [13].

\subsubsection{Signal to Noise Ratio}

Signal to noise ratio (SNR) is the amount of useable signal that is obtained relative to the amount of quantum mottle and scatter present in the image. The number of $\mathrm{x}$-rays per unit area $\mathrm{N}$ produced from a radiation source is statically characterize by the Poisson distribution and the variation in the number of $\mathrm{x}$-rays per area is the square root of $\mathrm{N}$. Therefore, the SNR is proportional to the square root of $\mathrm{N}$ and by extension the square root of the incident fluence $\phi_{i}$, the transmission factor of the patient $T$, the area of the detector element $A$ and the detection efficiency $\eta$. For a non homogenous material the difference in attenuation between the materials effect the ability to distinguish the useable signal from the noise and the scatter from the patient due to the Compton effect. Equation 1.2 shows the complete SNR for tissue of thickness $L$ composed of two components with attenuations $\mu_{1}$ and $\mu_{2}$; in addition, the equation also shows that the SNR has some dependence on the scatter fraction (SF) or the ratio of scattered x-ray photons reaching the detector compared to the total amount [13].

$$
S N R=\sqrt{A \varphi_{i} T \eta} \frac{2\left(1-e^{-L\left|\mu_{1}-\mu_{2}\right|}\right)}{\sqrt{1+e^{-L\left|\mu_{1}-\mu_{2}\right|}+\frac{2 S F}{1-S F}}}
$$

High SNR imaging with the current Electronic Portal Imaging Device (EPID) is not currently achievable at relatively low imaging doses due to low quantum efficiencies of between $2 \%$ and $4 \%$ [14] a usable image requires a SNR of at least 5 according to roses criteria for visibility. From Table 1.2 a SNR of 5 requires more than $1 \mathrm{cGy}$ when using a $2 \mathrm{MeV}$ x-ray source on a phantom containing a $1 \mathrm{~cm}$ thick bone in $20 \mathrm{~cm}$ of tissue [13].

\section{Table 1.2}

SNR values for various doses at therapeutic energies compared to the SNR from a diagnostic source [13]. 


\begin{tabular}{lcclcc}
\hline \hline Energy & $\begin{array}{c}\text { Diagnostic } \\
(50 \mathrm{keV})\end{array}$ & $\begin{array}{c}\text { Therapeutic } \\
(2 \mathrm{MeV})\end{array}$ & $\begin{array}{c}\text { Therapeutic } \\
(2 \mathrm{MeV})\end{array}$ & $\begin{array}{c}\text { Therapeutic } \\
(2 \mathrm{MeV})\end{array}$ & $\begin{array}{c}\text { Therapeutic } \\
(2 \mathrm{MeV})\end{array}$ \\
\hline $\begin{array}{l}\text { Patient } \\
\text { dose }\end{array}$ & $0.05 \mathrm{cGy}$ & $0.05 \mathrm{cGy}$ & $1 \mathrm{cGy}$ & $10 \mathrm{cGy}$ & $55 \mathrm{cGy}$ \\
SNR & 71 & $<1$ & 4.8 & 15 & 35 \\
\hline \hline
\end{tabular}

\subsubsection{Detector Efficiency and Quantum Efficiency}

Detector efficiency has two classifications, absolute efficiency and intrinsic efficiency. Absolute efficiency is the ratio of emitted signal or radiation from a source that the detector can capture and turn into an electric signal. It is dependent on the position and the size of the detector as well as the thickness. While the intrinsic efficiency is the ratio of the emitted signal reaching the detector that is detected. For $\mathrm{x}$-ray detectors this only depends on how much of the radiation is attenuated and the signal conversion process [15].

While detector efficiency is useful, to fully gage an imaging detectors capabilities detective quantum efficiency (DQE) is used. DQE measures the efficiency that the detector faithfully captures the input signal. Equation 1.3 shows DQE is the ratio between the SNR at the detector output to the SNR at the detector input [14].

$$
D Q E=\frac{S N R_{o u t}^{2}}{S N R_{\text {in }}^{2}}
$$

Equation 1.3

The detective quantum efficiency is a complete characterization of system performance and is dependent on spatial frequency, detector signal and detector fluence. Equation 1.4 shows the DQE expressed as a ratio between the modulation transfer function MTF, a measure of spatial resolution, noise power spectrum (NPS), related to the measure of white noise detected, for a mean detector signal $\bar{d}$ and incident $\mathrm{x}$-ray fluence $\bar{q}[14,12]$.

$$
D Q E(f)=\frac{\bar{d}^{2} M T F^{2}(f)}{\bar{q} N P S(f)}
$$


Where NPS is the noise power spectrum which can be found using equation 1.5.

$$
N P S(u)=\lim _{X \rightarrow \infty} \frac{1}{X}\left|\int_{X} \Delta d(x) e^{-i 2 \pi u x} d x\right|^{2}
$$

For the purposes of IGRT and when soft tissue contrast is required then a minimum of $20 \%$ DQE is required. The DQE of a MV flat panel imagers is currently around $1 \%$. [17]

\subsubsection{Measurement of Spatial Resolution}

Spatial resolution is directly measured using the point spread function (PSF) and the line spread function (LSF). In terms of CT imaging, the point spread function is measured using a pencil beam incident on the detector. Equation 1.6 shows the signal distribution, $P(x, y)$, created by the pencil beam is convertible into the LSF, $L(x)$, by integrating the signal in one direction $[12,16]$.

$$
L(x)=\int_{-\infty}^{\infty} P(x, y) d y
$$

Equation 1.6

The LSF can be directly used to measure spatial resolution using the full width at half maximum (FWHM). The FWHM can be used to measure how closely spaced objects can be placed and remain distinguishable from one another. Figure 1.4 illustrates that objects of the same composition can be placed up to one FWHM apart before they become indistinguishable from each other. Spatial resolution is formally measured with the modulation transfer function (MTF) as a function of line pairs per unit distance. From the LSF the MTF is obtained through the Fourier transform to convert the $L(x)$ into MTF(f) (Equation 1.7) $[12,16]$.

$$
M T F(f)=\int_{-\infty}^{\infty} \exp (2 i \pi f x) L(x) d x
$$




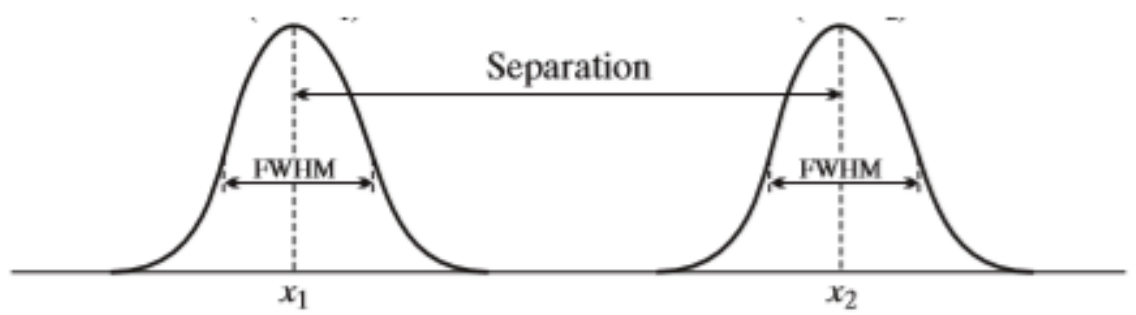

(a)

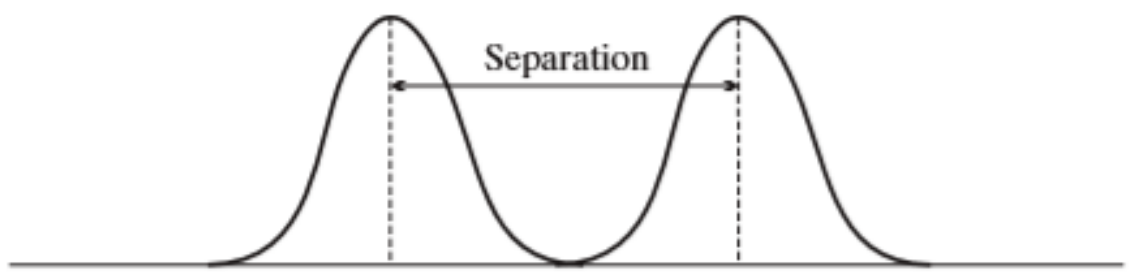

(b)

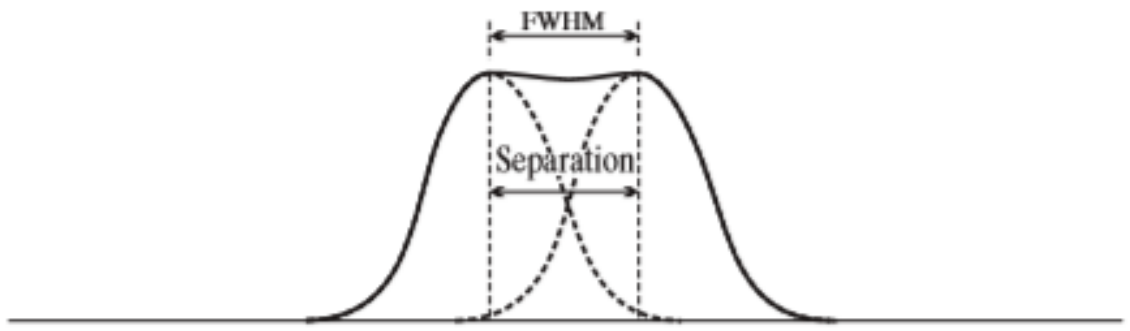

(c)

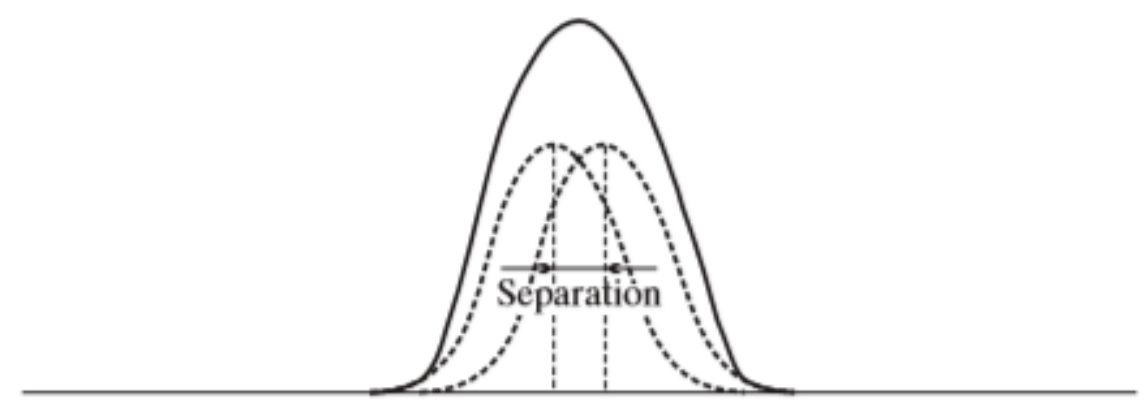

(d)

Figure 1.4 An example of how spatial resolution is ultimately calculated. Spatial resolution depends on how close two object can be from each other and still be distinguishable from one another [16]. 
The MTF is a measure of the contrast of the signal. When the MTF has a value of 0.5 it is said that the contrast of the object is reduced to half. Likewise, when the MTF is zero the signal has no contrast. The value first resulting in a MTF values of zero is known as the cut off frequency. Figure 1.5 demonstrates the clarity of a signal for different MTF values. It is an effective way to compare two different imaging system, if the curves are of similar shape and have the same cut off frequency then it can be concluded that the two imaging systems are the same [16].

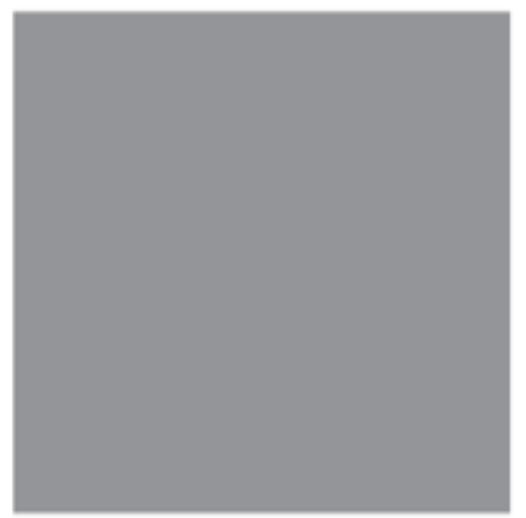

$m_{f}=0$

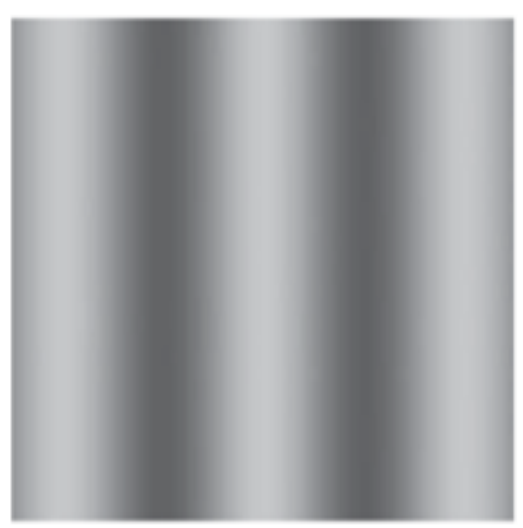

$m_{f}=0.5$

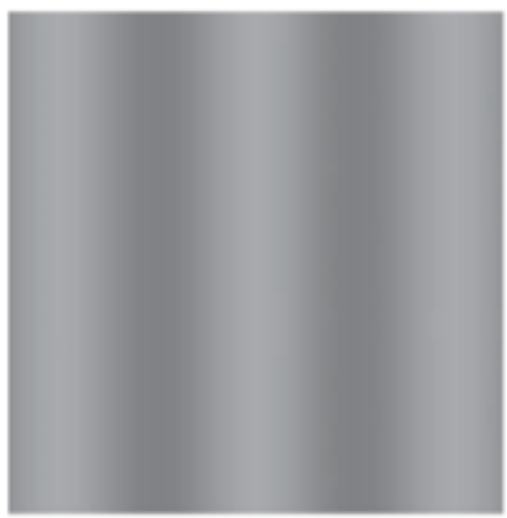

$m_{f}=0.2$

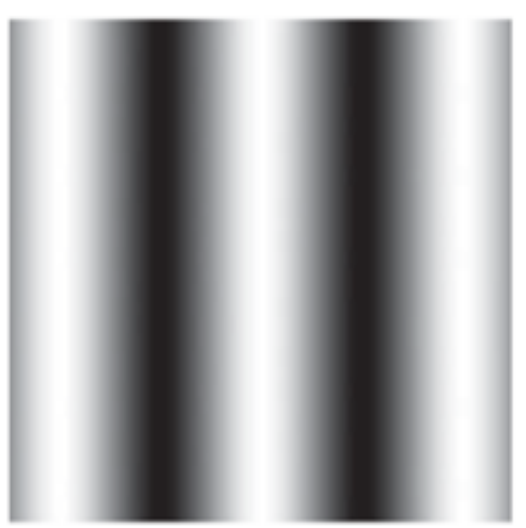

$m_{f}=1$

Figure 1.5 An example of the contrast differences between MTF values. The lines become less defined as the MTF value is reduced [16]. 
1.2.2.4 The Discrete Modulation Transfer Function, the Discrete Fourier Transform and the Fast Fourier Transform

Digital signal processing is currently used to evaluate many signal outputs in a rapid fashion. Digital signal processing of a LSF is done using the discrete Fourier transform (DFT) over coming the need to integrate over an infinite range of values. The DFT is numerically computable allowing it to be performed with a computational system for a sequence of values $x(n)$ from $0<n<N-1$. Equation 1.8 shows the DFT as a sum of a periodic function for a single spatial frequency $k$. This function is repeated for every spatial frequency from $0<\mathrm{k}<\mathrm{N}-1$. Large sets of data are difficult to handle with the DFT method requiring $\mathrm{O}\left(\mathrm{N}^{2}\right)$ operations. To use larger data sets a faster algorithm called the fast Fourier transform (FFT) which takes advantage of the symmetries in the DFT is used which reduces the number of operations down to $\mathrm{O}(\mathrm{N} \ln (\mathrm{N}))$ operations [18].

$$
X(k)=\sum_{n=0}^{N-1} x(n) e^{-j \frac{2 \pi}{N} k n}
$$

Equation 1.8

\subsubsection{Contrast and Contrast Noise Ratio}

Contrast is defined at the difference in signal between the two adjacent tissues or structures as seen in Equation 1.9 [12]. Contrast is dependent on the energy of the x-ray used for imaging. The contrast for MV imaging is low since, unlike KV imaging where the photoelectric effect is dominant, MV imaging instead relies almost entirely on the Compton effect to generate contrast [14].

$$
C_{12}=\left|S_{1}-S_{2}\right|
$$

Where $C_{12}$ is the difference in contrast between material 1 and 2. [12]

The contrast generated between two materials using the Compton effect is from the difference in electron density and the density of the material itself. For soft tissues the difference is small [14]. The contrast to noise ratio is used to determine if the imaged objects are resolvable from one another. In other words, the signal to noise ratios between the two tissues must be different enough to be discernable from one another (Equation 1.10) [12]. 


$$
C N R_{12}=\frac{C_{12}}{\sigma_{N}}=\frac{\left|S_{1}-S_{2}\right|}{\sigma_{N}}=\left|S N R_{1}-S N R_{2}\right|
$$

Where CNR is the contrast to noise ration between materials 1 and 2 and $\sigma_{N}$ is the standard deviation of the noise. The CNR is calculated using the difference between the SNR between materials 1 and $2[12]$.

\subsection{The Types of Electronic Portal Imaging}

Megavoltage X-ray imaging is performed using electronic portal imaging devices (EPID) with active matrix flat panel imagers (AMFPI). Two general types of EPID-AMFPI were developed: indirect detection and direct detection. Indirect detection uses a metal plate and scintillator combination to convert $\mathrm{x}$-rays into optical light. The optical light is later converted into electrons using a photodiode (figure 1.6a). Direct detection detectors use a combination of a metal buildup plate and a photoconductor coupled directly to the capacitor built into each pixel (figure 1.6b) [14].

(a)

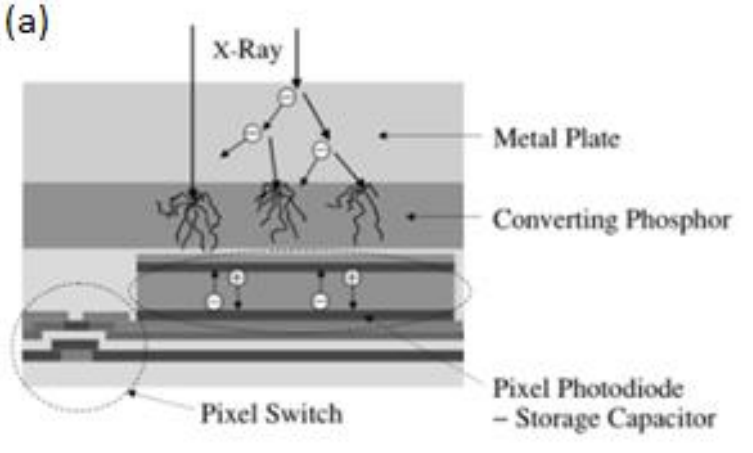

(b)

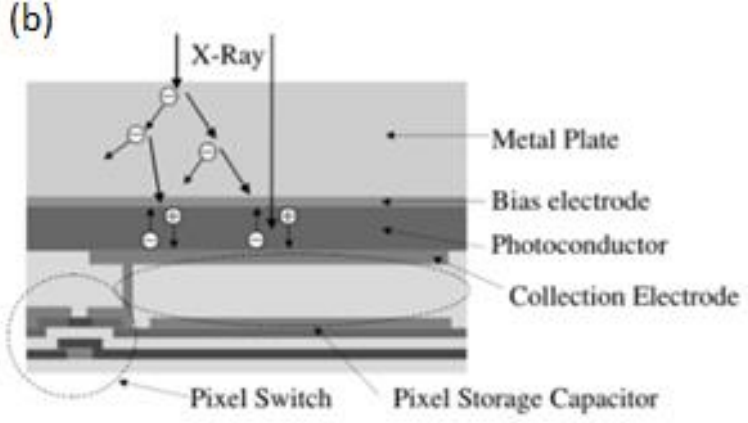

Figure 1.6 An illustration of two types of X-ray detection method for portal imaging. (a) shows the indirect method which uses a converting phosphor and photodiode to generate and signal. (b) shows the direct detection method where the generated electrons are collected. A photoconductor with a collection electrode is used to generate a signal. [14] 


\subsection{Early Improvements to Electronic Portal Imaging}

\subsubsection{Indirect Detection}

The commercial release of EPID-AMFPI detectors was mostly based on indirect detection with a few variations in design such as using different scintillating materials like phosphor or CsI(TI). The advantage of this type of system is the variety of scintillators that can be used. The scintillators can be segmented with light reflecting spacers like those found in a positron emission tomography imaging device to isolate each detector element. This allows for a thicker "phosphor" layer without degrading the spatial resolution. Theoretically the maximum quantum efficiency ( $Q E$ ) can be $100 \%$ however practically this cannot be obtained without exceedingly thick detectors [19]. Borrowing concepts from previous imaging modalities such as the use of septa in positron emission tomography to reduce scatter between elements [20] this segmented phosphor design also had some anti-scatter properties. As shown in figure 1.7 the segmented phosphors run radially outward, parallel to the incident beam of a LINAC. The advantage to this design is the ability to increase the phosphor thickness without losing spatial resolution from optical light spreading throughout the phosphors. This design achieved a high QE (>50\%) with a few centimeters of material [19]. 


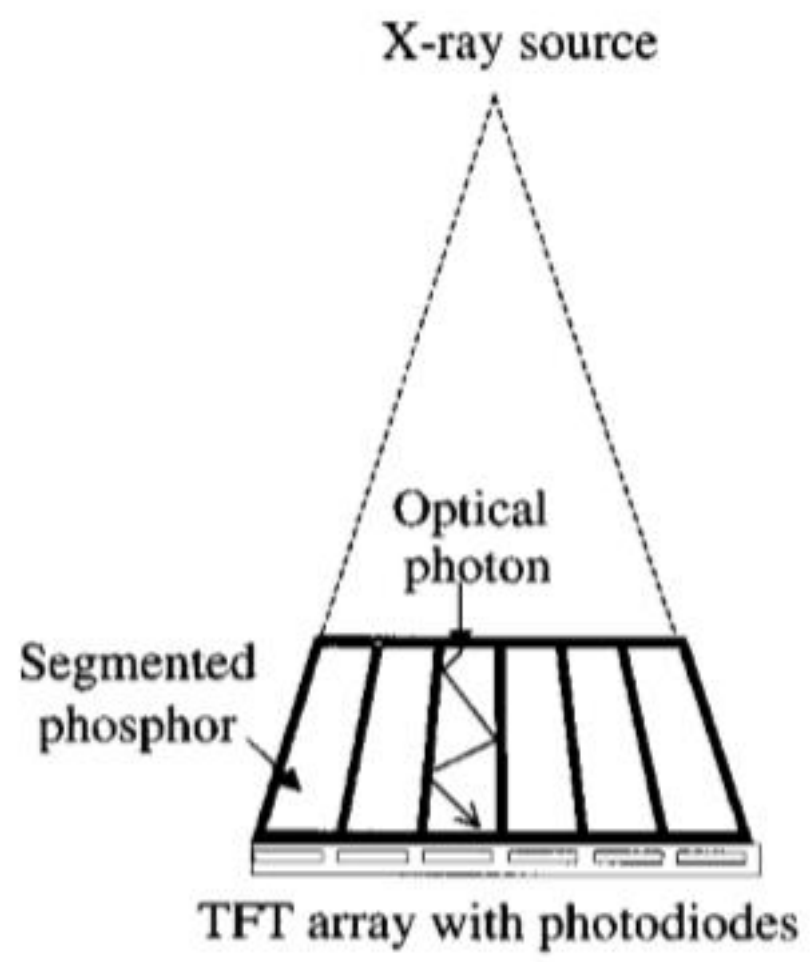

Figure 1.7 An early idea for a high quantum efficiency indirect detection type portal imager. The elements are aligned with the x-ray source for an unknown field size [19].

\subsubsection{Direct Detection}

This design was soon altered to create a direct conversion detector. In 2004 a paper was published with a similarly designed detector except instead of using phosphorus material [21], a different approach using metal spacers was made [22]. Metals are particularly good in the role of the conversion layer, converting the x-ray to high energy electrons. Metals such as copper and tungsten where used in previous design ideas yielding quantum efficiencies as high as $60 \%$ for either $3.6 \mathrm{~cm}$ of copper or $1.3 \mathrm{~cm}$ of tungsten [19]. Similarly, to the phosphorus and spacer design in figure 1.7, layers of plates separated by readout electrodes were arranged as shown in figure 1.8 [22]. 


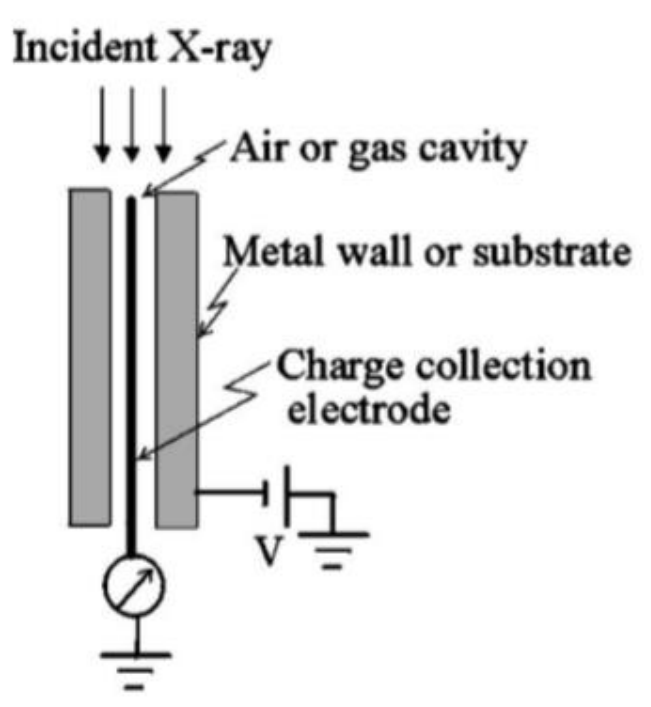

(a) Single element

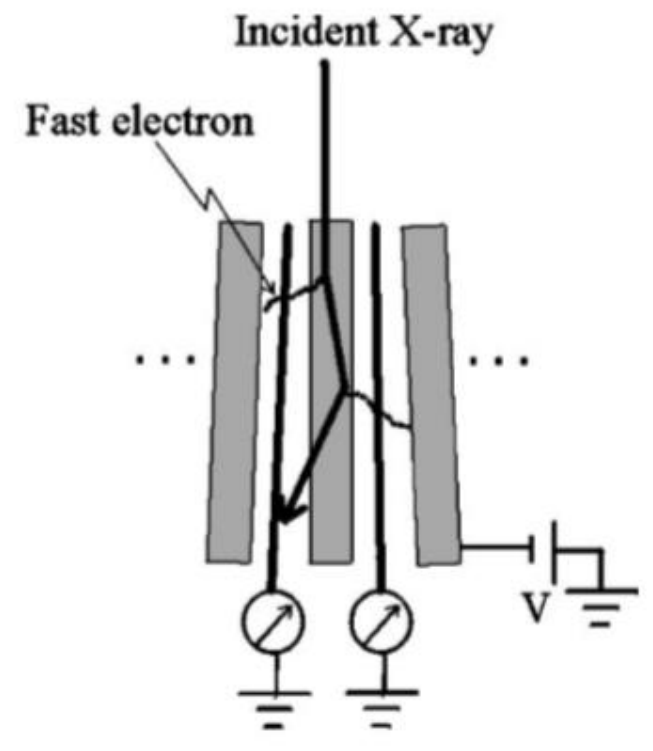

(b) Multiple elements

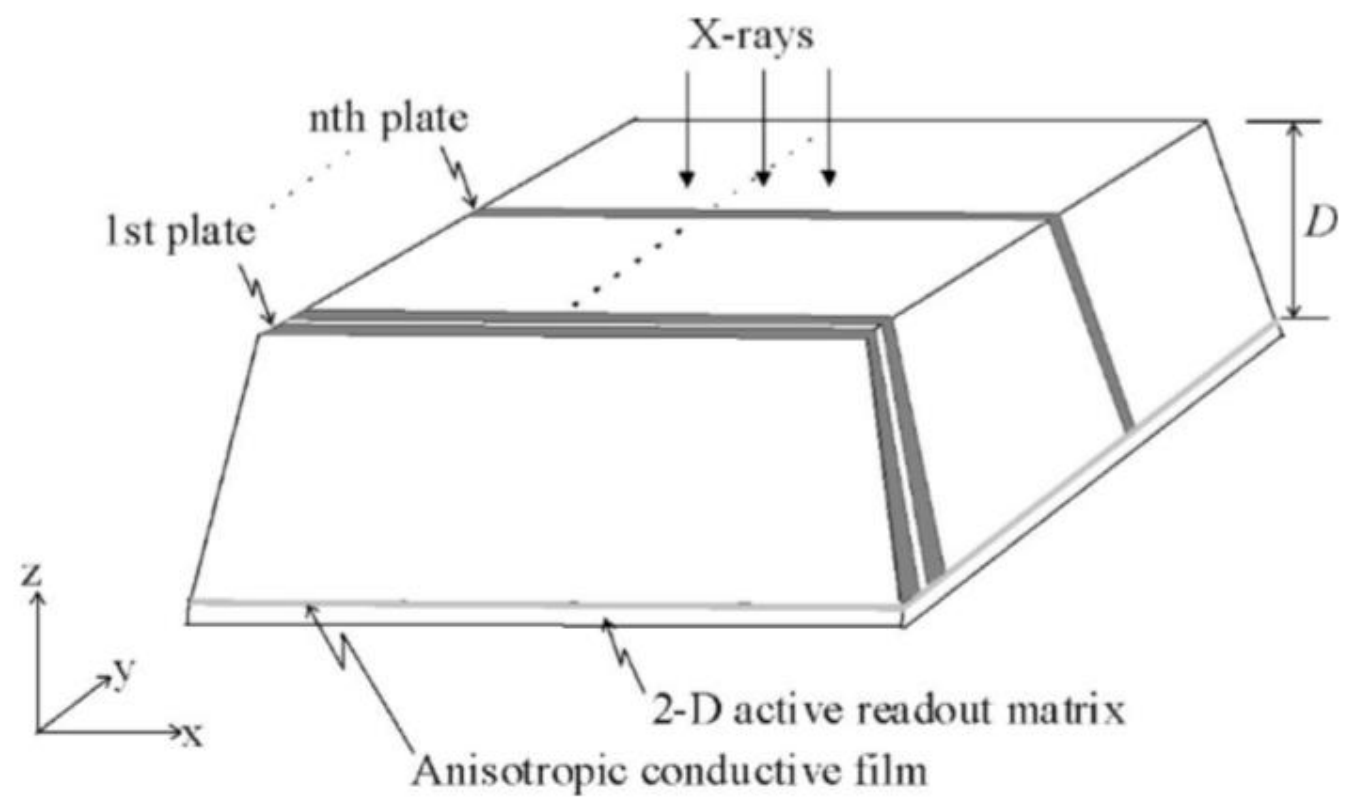

Figure 1.8 An early design of a direct detection portal imager. Top (a) shows the composition of a single element and (b) shows multiple elements, they are arranged in a trapezoidal configuration like the previous design. Bottom shows a full detector. [22] 
Each electrode was spaced out by metal spacers creating a $40 \mathrm{~cm}$ by $40 \mathrm{~cm}$ grid of readout electrodes. This design was not only capable of high quantum efficiency ( $>50 \%$ with a few $\mathrm{cm}$ of tungsten or copper) but also equivalent spatial resolution (figure 1.9) to existing flat panel imagers at the time [22, 23]. Despite its advantages over the technology at the time a large scale detector of this type proved to be difficult and expensive to build.

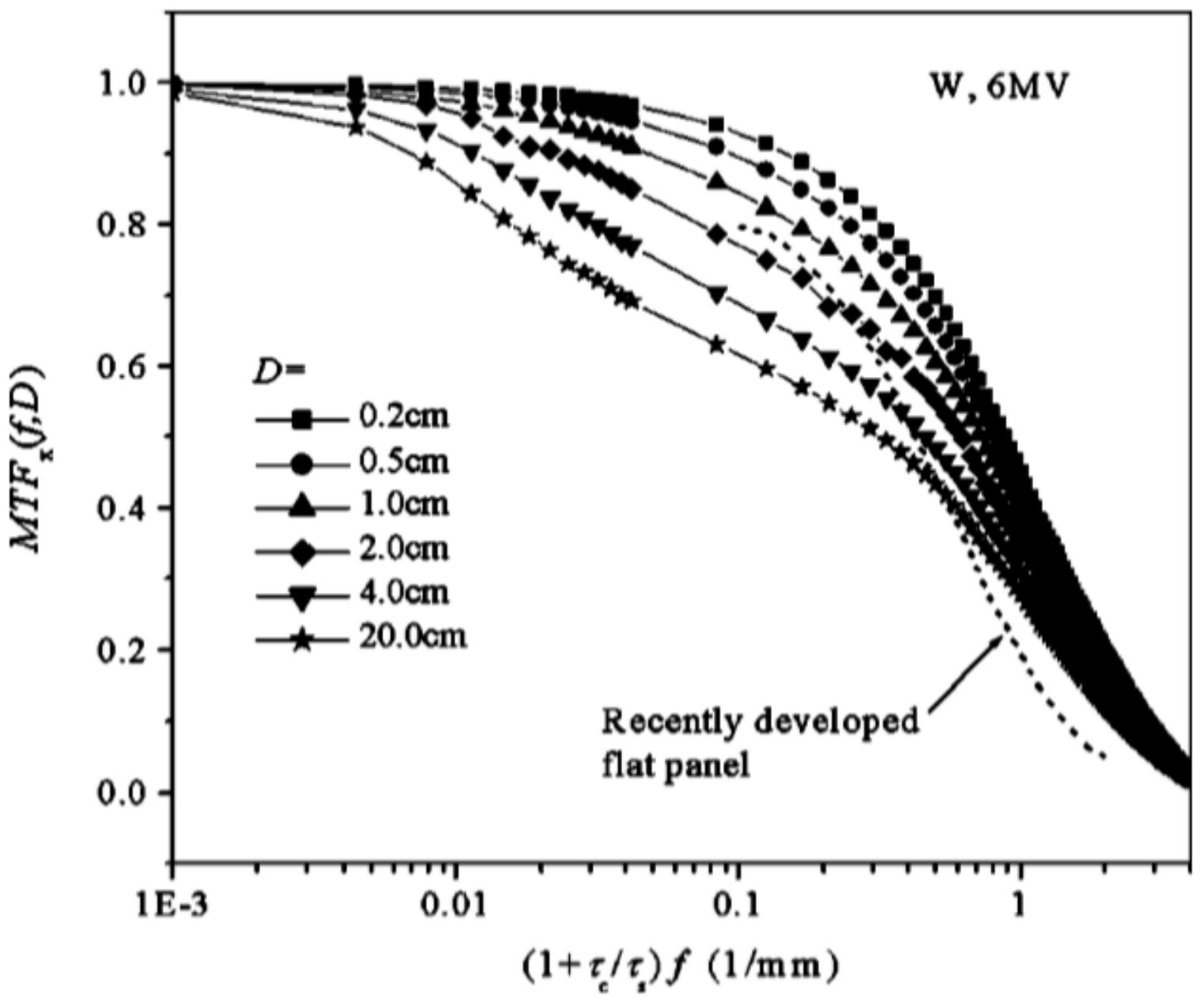

Figure 1.9 The spatial resolution measured via MTF for the detector shown in figure 1.8. The thinnest detector appears to consistently have the higher spatial resolution [22]. 


\subsection{The Cerenkov Portal Imaging Device}

Indirect detection was revisited to find an inexpensive and easy to build detector capable of similar spatial resolution and high QE in comparison to the clinically used EPIDs. One method that was of particular interest was the use of optical fibers to detect high energy $\mathrm{x}$-rays. Optical fibers can be used to detect Cerenkov radiation produced from an electron generated by $x$-rays travelling faster than the speed of light in the fibers. This gives the detector inherent anti-scatter properties since the criteria for Cerenkov generation is given by Equation 8. A signal would only be obtainable by $\mathrm{x}$-rays with energies high enough to generate electrons with higher speed relative to the speed of light $(\beta)$ in a medium with a refractive index $\mathrm{n}[24,25]$.

$$
n \beta>1
$$

Equation 8

The light output from the Cerenkov effect is low, two orders of magnitude lower than $\mathrm{Cu} /$ phosphor screen combination [24], requiring optical fibers to transmit the light without much loss for thicker detectors. The Cerenkov detector consisted of a grid of optical fibers embedded in an optically reflective material to prevent leakage of light from one fiber to another. The fibers were aligned to be parallel to the incident $\mathrm{x}$-ray beams for the Monte-Carlo simulations. The reflective material chosen for this application was opaque resin. The choice of material gave the detector a low Z-value to prevent over response from low energy $\mathrm{x}$-rays, as well as anti scatter properties since the lower energy Compton scattered $x$-rays would be less likely to create a signal in the material. The lower scatter fraction would ultimately increase the SNR in accordance to equation 6 . The design of this detector can be seen in figure 1.10 , here the detector only replaces the copper buildup layer and the phosphorus screen from figure 1.6(a). This methodology of using Cerenkov light instead of scintillator light lead to the imager being called the Cerenkov portal imaging detector (CPID). Figure 1.11 shows the CPID designs spatial resolution has a low dependence on thickness in comparison to the previous direct detection design for a $6 \mathrm{MV}$ beam. Since spatial resolution was not strongly dependent on the thickness of the detector, the detector could be made as thick as desired to increase the quantum efficiency with a QE of $67.1 \%$ at $30 \mathrm{~cm}$ thick for a 6 MV beam [25]. As mentioned previously it was advantageous for the portal imager to be stowed away during setup to make it easier to position the patient and then bring it out when necessary [14]. For this reason, a more compact detector would likely be necessary to develop in order to replace the currently used system with minimal redesign of the LINAC. 


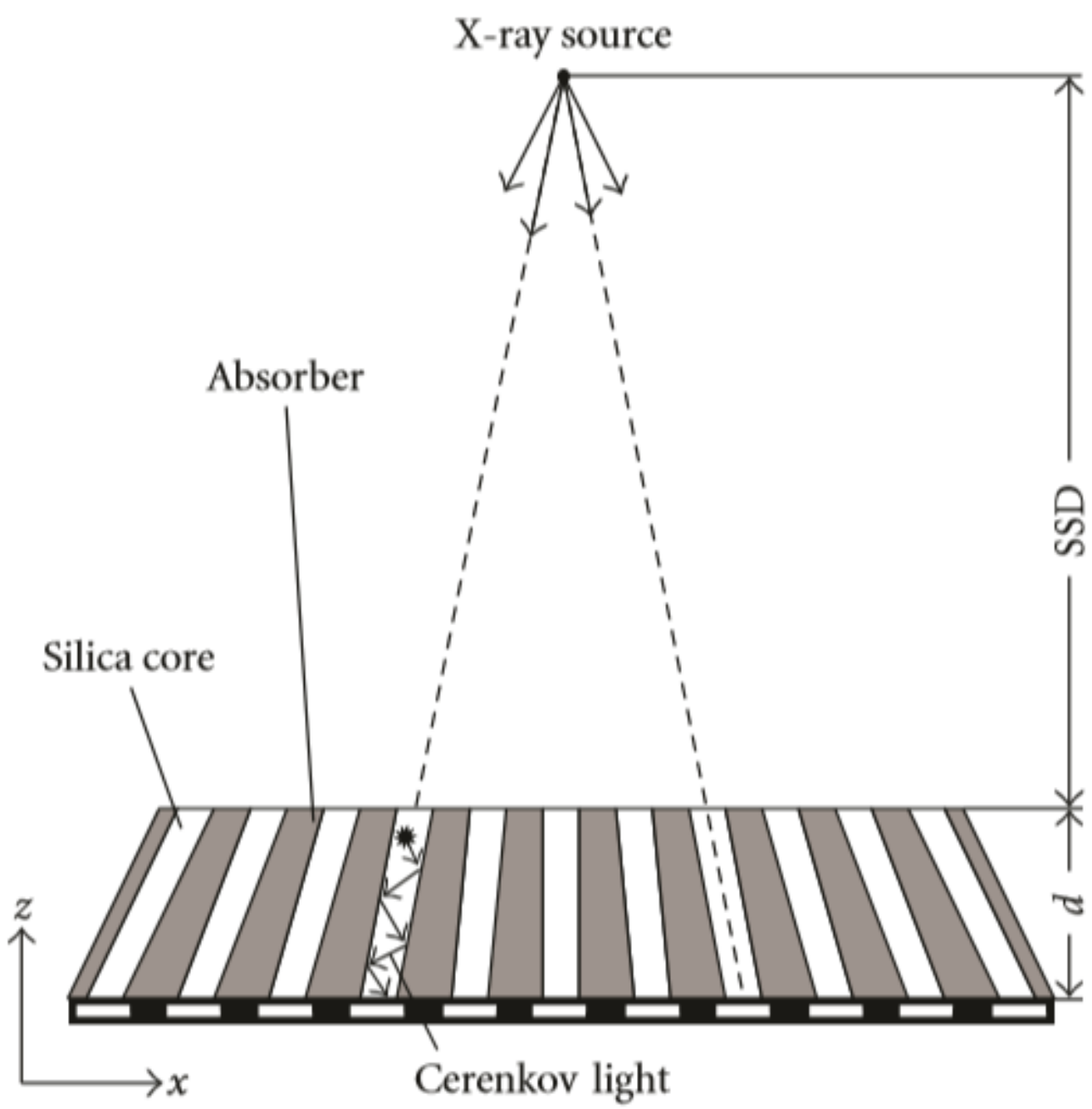

Figure 1.10 An indirect portal imager that relies on Cerenkov radiation to convert the incoming $x$-rays into optical photons via the generated super luminal (relative to the medium) electrons. The absorber is an opaque black resin with similar properties to water. The fibers are again slanted towards the x-ray source [25]. 


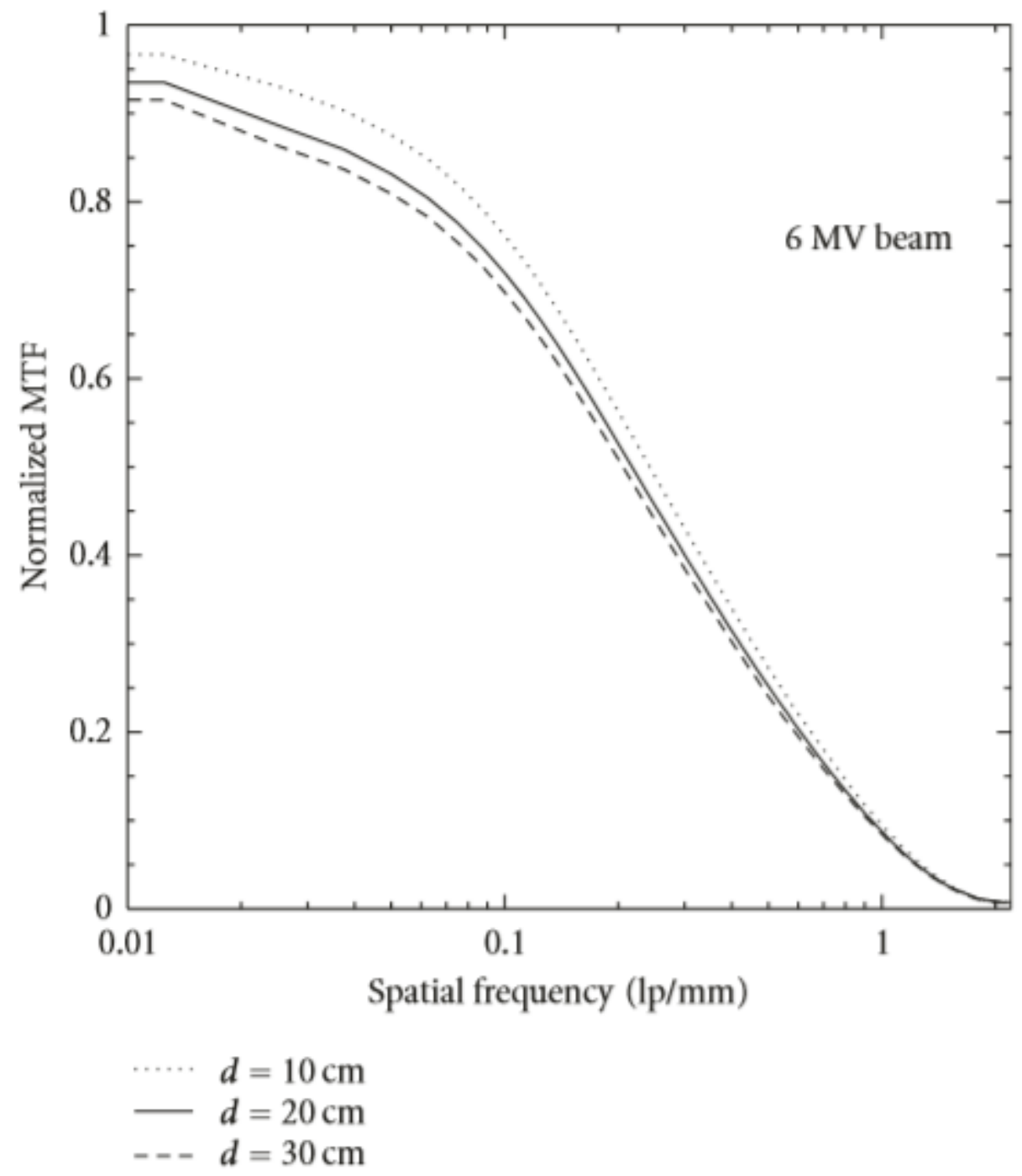

Figure 1.11 The measured spatial resolution for the detector described in figure 1.10. The thickness of this detector does not appear to significantly affect the spatial resolution over the range of spatial frequencies shown [25].

The first attempt at making a more compact detector was done by changing the type of optical fibers in the CPID. The goals of this attempt were two-fold. The first goal was to increase the collection efficiency of Cerenkov photons, since the optical fibers used has a low numerical aperture and very few optical photons were created per event, very few Cerenkov photons were able to reach the AMFPI. This would require an AMFPI with avalanche gain which was not available at the time. The second goal was 
to make the CPID more compact so it could be more easily stowed away when not in use while not sacrificing quantum efficiency and spatial resolution [26].

The idea to maximize the light transmission through the optical fiber was done by increasing the acceptance angle $\theta$ of the fiber. All fibers consist of two components, a core where the light is transmitted down the length of and one or more claddings, which envelops the length of the fiber, to keep light internally reflected. Every fiber has a cladding either by design or simply from their surroundings; in the latter case the cladding can either be very poor or very good depending on the refractive index $\mathrm{n}$. From equation 9 the optical transmission efficiency can be increased by picking a fiber with a cladding with a much lower refractive index than the core [26].

$$
\theta=\frac{\pi}{2}-\arcsin \left(\frac{n_{\text {clad }}}{n_{\text {core }}}\right)
$$

In theory the ideal cladding for any optical fiber would be vacuum which would have a refractive index of 1; however, air turns out to be a close substitute for this with a refractive index of $\sim 1$. Therefor by cladding the optical fibers in air it would be possible to make the detector more efficient at collecting a signal [26].

To minimize the bulkiness of the detector denser optical mediums were considered such as lead glass. Using lead glass instead of the original fused silica fibers made it possible to reduce the detectors thickness to less than a third of its original thickness while maintaining the same quantum efficiency and incidentally improving the spatial resolution of the detector from a $50 \%$ MTF value of $0.18 \mathrm{Ip} \mathrm{mm}^{-1}$ for silica fibers to a $50 \%$ MTF value of $0.36 \mathrm{Ip} \mathrm{mm}^{-1}$ which was above the performance of the video based EPIDs at $0.2 \mathrm{lp} \mathrm{mm}^{-1}[26]$.

\subsection{Geant4 and Monte Carlo Simulations}

Modern detector design requires the use of computational methods to mimic the physics of radiation interactions and the responses of the detector. One such method is through Monte-Carlo simulation packages which utilize random processes tempered by the theoretical equations that govern the various physical interactions between high energy particle and matter. Geometry and Tracking 4 (Geant4) is a toolkit developed for uses such as detector design, high energy physics and space science. Such simulations require a deeper knowledge of the materials and mechanisms in play in order to successfully mimic a radiation detector[27]. 
Monte Carlo simulations have become widespread in medical imaging to measure values that are difficult to measure experimentally. For clinical applications the reliability of the Monte Carlo code is important. Reliability of the simulation is obtained by comparing it against an empirical test such as an experiment. This requires extensive knowledge of the conditions of the experiment. For validation it is important that the simulation conditions are simple enough to be reconstructed and the measurements general enough to obtain a reasonable measure of the detectors' performance [28].

One strategy for simulation validation, especially for new Monte Carlo code installations, is to first obtain validation against a database such as NIST for the physical processes of interest such as attenuation cross sections. This ensures that the Monte Carlo code has been installed without relevant errors and allows the user to compare physics packages and their accuracy in comparison to the NIST database for their purposes. After checking the basic physics processes it is useful to compare to a similar simulation to the experimental setup that has already been validated. This ensures that the simulation environment has been setup correctly and the more advanced physical processes, such as the Compton scatter distribution and energy distribution, under various situations like the measurements to be performed for an experimental validation. Finally, experimental validation can be attempted providing that the environment is reasonably matched [28].

\subsection{The Scintillating Fiber Portal Imaging Device}

A new detector prototype was obtained from a spare piece of a barrel calorimeter. It was composed of lead and plastic scintillating fibers. The plastic scintillating fibers operate similarly to optical fibers with a portion of the optical light being trapped in the fibers until reaching one of the ends. Since the fibers were capable of scintillation the collection efficiency could be improved, with a sacrifice of some of the inherent anti scatter properties, over the previous CPID design. With lead as a filler material the overall density of the material was higher than the resin and lead fiber design. The plastic scintillators utilize the organic scintillation mechanism involving molecular excitation states instead of the semiconductor excitation states found in inorganic scintillators [15]. 


\subsubsection{Organic Scintillation}

Organic scintillation occurs from transitions in the energy level structure of molecules and is independent of physical state. They can be easily molded and shaped into any form, such as long fibers, without losing their scintillation properties. The energy of an incoming charged particle is absorbed through the excitation of the electrons within the structure of the scintillator. The electrons can be excited into two type of states, singlet and triplet spin states. The excitation states are illustrated in figure 1.12 where the singlet spin states are labelled as $S_{0}, S_{1}, S_{2}$, and so on while the triple states are labelled as $T_{1}$, $T_{2}, T_{3}$ etc. For organic scintillators the energy difference between $S_{1}$ and $S_{0}$ is usually between 3 and $4 \mathrm{eV}$. Each transition has several smaller states within called vibrational states which are usually represented by a second subscript. If the electron enters a state higher than $S_{1}$ the electron quickly de-excites into the $S_{1}$ state in the order of picoseconds through electron capture and therefore does not emit radiation. A similar de-excitation is also observed for vibrational states. These radiation-less de-excitations are termed as quenching. Only when the electron reaches the $S_{1}$ state does it de-excite with a photon emission, this transition typically takes on the order of nanoseconds. Triplet states occur through a transition called intersystem crossing where an excited singlet state may transition into a triplet state. The $T_{1}$ state like the $S_{1}$ state also transitions into the $S_{0}$ state through the emission of a photon through phosphorescence which may be on the order of milliseconds [15].

Organic scintillators require more energy to excite the electrons than they emit when de-exciting allowing them to be mostly transparent to their own fluorescence emission. This is illustrated in figure 1.12 where the length of the arrows represents the amount of energy required for each transition. The difference in absorption and emission spectra is called the Stokes shift. Because the emission and absorption spectra don't overlap significantly this means that the scintillating fibers can operate similarly to optical fibers. By choosing fibers with the proper cladding the optical light that doesn't get reabsorbed can be efficiently transferred to the AMFPI; furthermore, more light can be generated per event. Occasionally the de-excitation energy is incompatible with the photodiode or semiconductor material used in photodetectors to convert the optical light into charge. To make the scintillator more compatible an addition of a component known as a wave shifter is added to the mixture. A wave shifter absorbs the radiated light from the parent material and remits it as another wavelength. Plastic scintillators are composed of a polymer of scintillating material dissolved in a solvent such as styrene. As a plastic it is possible to form large area detectors without much cost and in a similar fashion to optical fibers these can 
be coated with a cladding layer to increase transmission efficiency. Plastic scintillators are commonly used in the field of high energy physics as large volume detectors in bundles of fibers. In this application they are generally subjected to large amounts of dose on a regular basis. The typical plastic scintillator is not resilient to high doses and will often experience significant degradation for exposures in the range of 1 to $10 \mathrm{kGy}$; however, there exist radiation resistant formulations that show little degradation under doses as high as $100 \mathrm{kGy}$. Degradation usually results in the loss of light output by either damage to the fluorescent component or by the creation of absorption centers in the fiber [15]. The fibers used in the prototype detector (Kuraray SCSF-78M, 0.5mm, double cladding) were capable of withstanding $10 \mathrm{kGy}$ without showing significant signs of damage [38].

Plastic scintillators are generally very good for the detection of beta and other charged particles and poor at the detection of high energy photons. This will result in the generation of only the Compton continuum in a measured spectrum with almost no photopeak. While it is possible to dope the scintillators with higher $Z$ material such as tin, this doping will lead to less light output from the scintillators since the impurities will absorb the optical light in the fibers and poorer energy resolution. The response of organic scintillators to charged particles is described by the amount of fluorescent energy emitted per unit path length $(\mathrm{dL} / \mathrm{dx})$ and the amount of energy loss or stopping power $(\mathrm{dE} / \mathrm{dx})$. The relationship is best described by Birk's formula as shown in equation 1.11 with the introduction of the scintillation efficiency $S$ and an adjustment parameter $k B$ which varies depending on the scintillator [15].

$$
\frac{d L}{d x}=\frac{S \frac{d E}{d x}}{1+k B \frac{d E}{d x}}
$$


Singlet

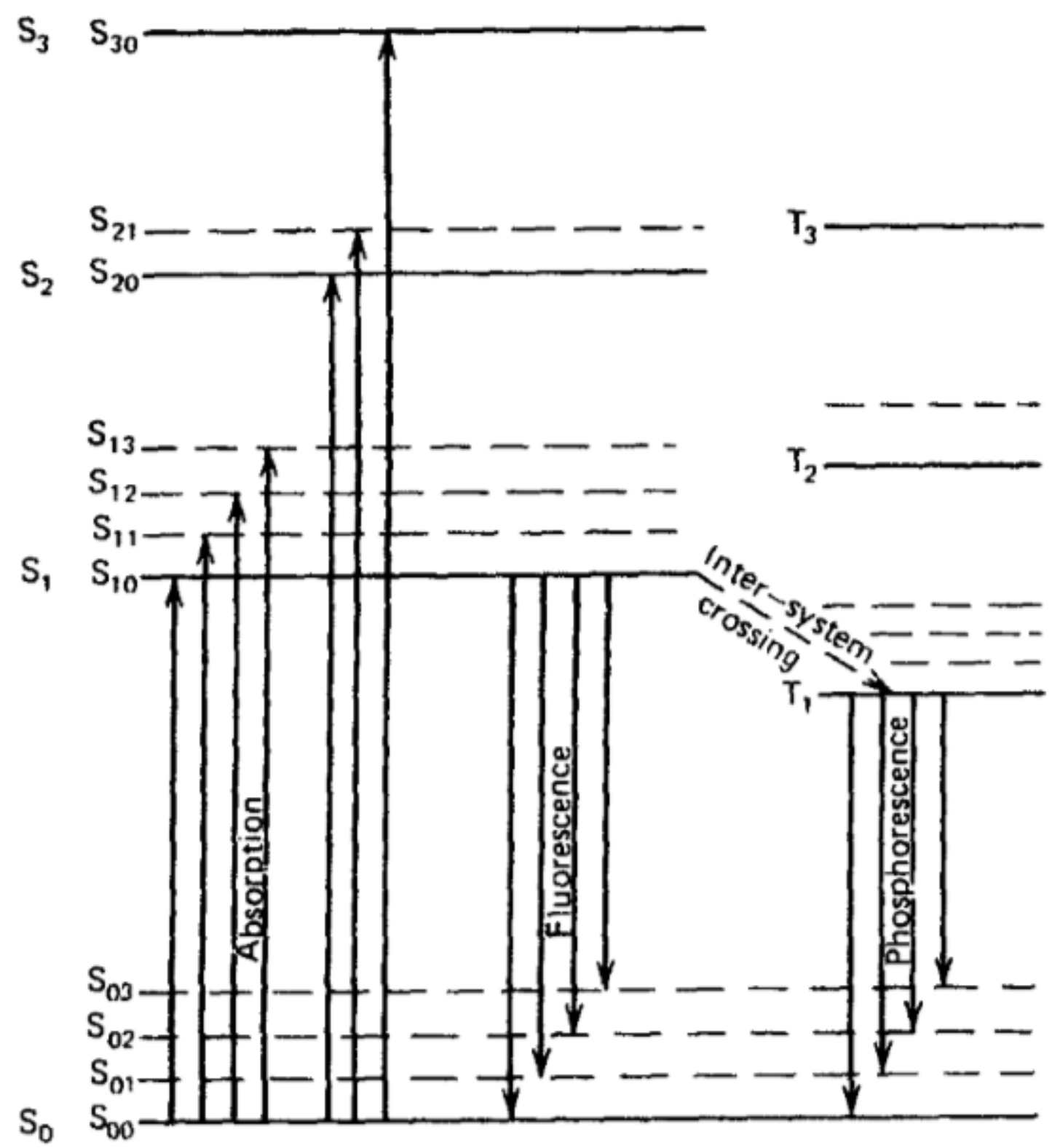

Figure 1.12 This figure shows the 'energy levels of an organic molecule with [a] $\pi$-electron structure'. In most cases the amount of energy to excite the molecule is greater than the amount of energy released as radiation when it relaxes [15]. 


\subsection{Objective}

Using all the knowledge of how a xray detector behaves and the physical interactions involved with photons and charged particles with matter it is possible to generate a Monte Carlo simulation of a prototype detector. From the simulation match of the prototype detector it would be possible to modify the detector without a physical copy of the device creating something entirely new.

The objective of this project was to develop a Monte-Carlo simulation of the prototype MV x-ray detector that was provided by the University of Regina using measurements of spatial resolution and scatter primary ratio acquired by Jian Liu [34]. A reasonably close match between the experimental results and the simulation results for total signal and MTF would signal a successful simulation. This will allow for further development of this version of the detector to optimize spatial resolution and maximize SNR while minimizing the amount of radiation needed to achieve the minimum value of between 5 and 7 in accordance with the Rose model. 


\section{Chapter 2}

\section{Methods}

\subsection{Materials}

The prototype detector was made by layering corrugated $0.5 \mathrm{~mm}$ thick lead foil with plastic scintillating fibers (Kuraray SCSF-78MJ, Kuraray Co., Ltd., Tokyo, Japan) into a hexagonal grid as shown in figure 2.1. The fibers and the lead were bonded together using BC-600 optical epoxy (Saint-Gobain Crystals, $\mathrm{OH}$, USA) which has some light transmission properties in the $400 \mathrm{~nm}$ wavelength. The Kuraray fibers are double cladded and built using the materials shown in table 2.1. Double cladding fibers were chosen due to their higher light trapping efficiency than single cladding. A higher trapping efficiency was useful for the original use of the detector as part of a $4 \mathrm{~m}$ long barrel calorimeter. This detector was used by Jian Liu to obtain all the experimental data used for the simulation match. The original data can be seen in his thesis paper [34].

\section{Table 2.1}

The materials used to make the fibers used for the prototype detector. The chemical formulas for the core and the inner cladding are well known but the formula for the outer cladding was a mystery. As a result, tetrafluoroethylene was used in the simulation. The material was forced to have the same density and refractive index as the outer cladding [34].

\begin{tabular}{llcc}
\hline \hline & Material & Refractive index, $\mathbf{n}$ & Density (g/cm $\mathbf{c}^{\mathbf{3}}$ \\
\hline Core & Polystyrene (PS) & 1.59 & 1.05 \\
\hline Inner Cladding & Polymethylmethacrylate (PMMA) & 1.49 & 1.19 \\
\hline Outer Cladding & Fluorinated polymer (FP) & 1.42 & 1.43 \\
\hline \hline
\end{tabular}

The hexagonal grid shown in figure 2.1 was modelled using the Geant4 Monte Carlo software using a lead volume with fibers and glue embedded in the structure. Cylinders of glue, cladding and core were constructed as daughter elements of the lead volume. The glue cylinder had an outer radius of 0.553 $\mathrm{mm}$ while the fibers had an outer radius of $0.5 \mathrm{~mm}$ according to figure 2.2 each layer of cladding occupied 
$3 \%$ of the total diameter. This leads to the cladding layers having a thickness of $0.015 \mathrm{~mm}$ each. The outer cladding is listed as a fluorinated polymer but without a formula attached, while the density was listed as $1.43 \mathrm{~g} / \mathrm{cm}^{3}$ a polymer with this density could not be found among a list of commonly used polymers. To build this fluorinated polymer the empirical formula was assumed to consist of fluorine and carbon atoms which is used for many of the commonly used fluoropolymers. For simplicity the formula $\mathrm{F}_{4} \mathrm{C}_{2}$, tetrafluoroethylene, was used and assigned the density of $1.43 \mathrm{~g} / \mathrm{cm}^{3}$. This was assumed to be appropriate since this fluorocarbon is primarily used in the preparation of polymers [37]. The refractive index for the outer cladding was set to 1.42 for all wavelengths. The inner cladding was composed of polymethylmethacrylate (PMMA) which was listed with the composition of $3.6 \times 10^{22}$ atoms $/ \mathrm{cm}^{3}$ carbon, $5.7 \times 10^{22}$ atoms $/ \mathrm{cm}^{3}$ hydrogen and $1.4 \times 10^{22}$ atoms $/ \mathrm{cm}^{3}$ oxygen. The empirical formula for PMMA was not deciphered from the formula given, instead a compound was created using a by weight approximation consisting of $60.6 \%$ carbon, $8 \%$ hydrogen and $31.4 \%$ oxygen. The core was composed of polystyrene which was among the predefined materials in Geant4. The glue was selected to be polyvinyl acetate from the list of Geant4 materials since the formula for the epoxy was not known but a density of $1.18 \mathrm{~g} / \mathrm{cm}^{3}$ was given in the brochure along with a refractive index of 1.56. Polyvinyl acetate has a density of 1.19 $\mathrm{g} / \mathrm{cm}^{3}$ which is like the epoxy, the refractive index was set to 1.56 for all wavelengths. 


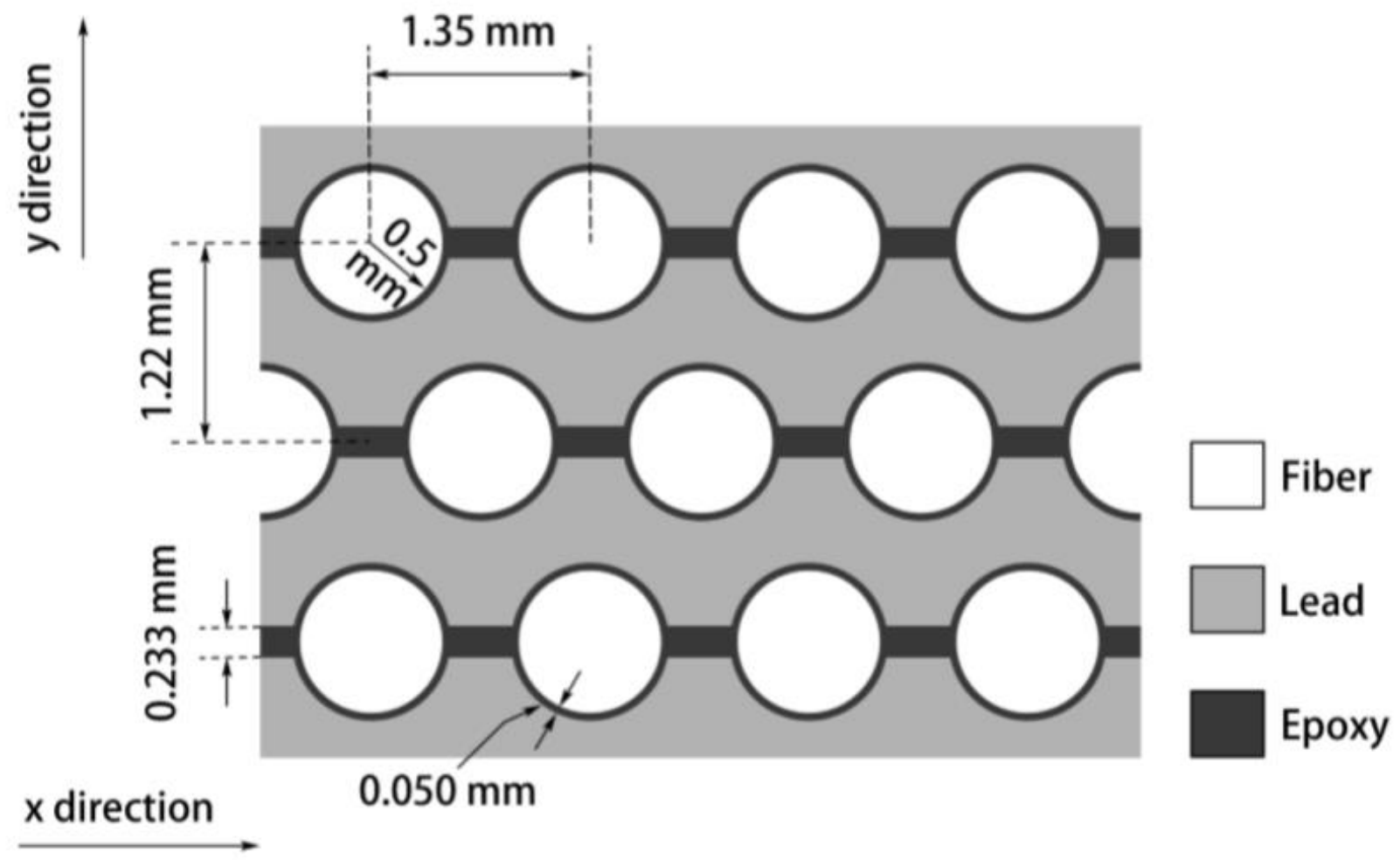

Figure 2.1 A close-up of the construction of the prototype detector. The Fibers use were plastic scintillating fibers (Kuraray SCSF-78MJ, Kuraray Co., Ltd., Tokyo, Japan) and the epoxy was BC-600 optical epoxy (Saint-Gobain Crystals, OH, USA). The components of the fibers are shown in table I and the geometry of the fibers is shown in figure 2.2. [34] 


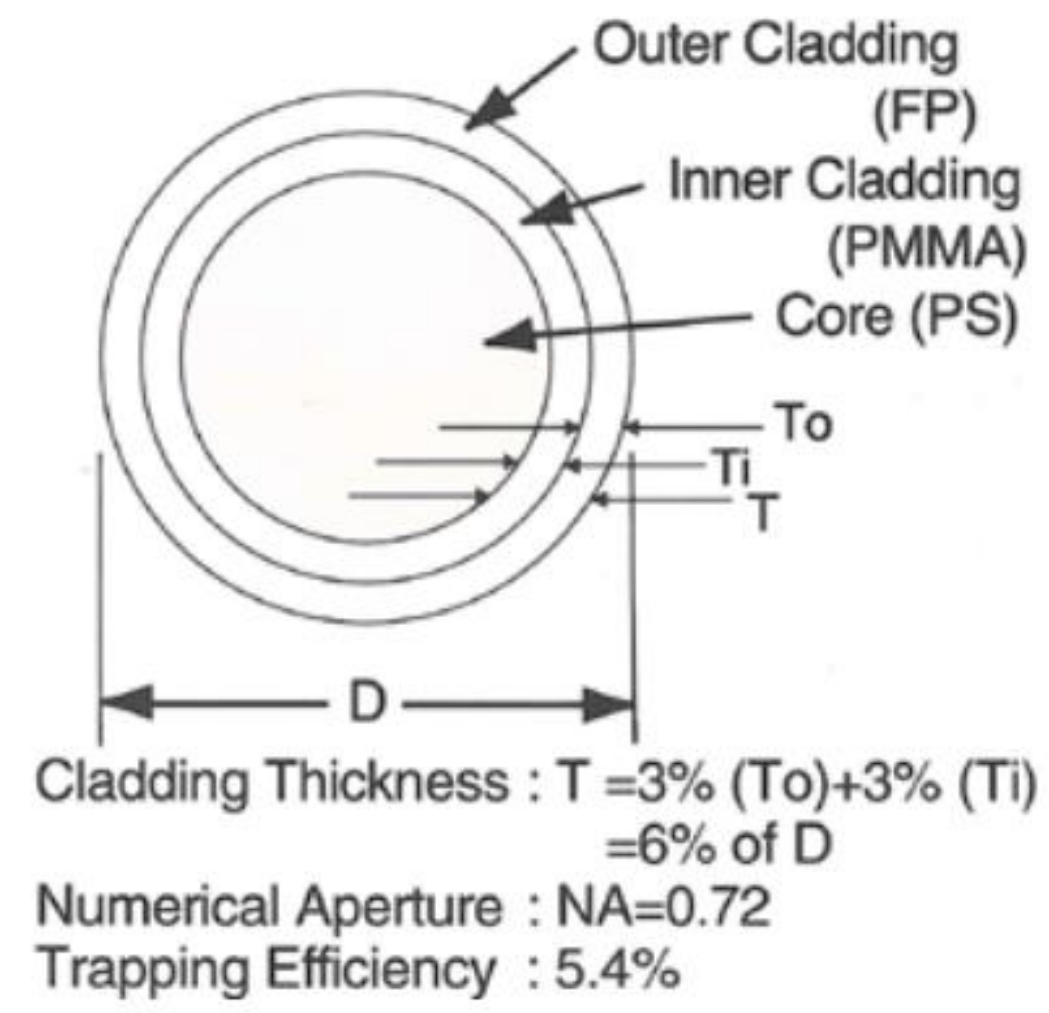

Figure 2.2 The geometry of the double cladded plastic scintillating fibers (Kuraray SCSF-78MJ, Kuraray Co., Ltd., Tokyo, Japan).

\subsection{General Experimental Setup}

The experimental setup consisted of a $7 \mathrm{~cm}$ by $12 \mathrm{~cm}$ by $2 \mathrm{~cm}$ detector with the central pixel replaced by a much longer scintillating fiber coupled to an optical fiber. The fiber itself was $10 \mathrm{~m}$ long and lead to a spectrometer for readout. The detector was set parallel to the table with the LINAC gantry angle set to 90 degrees such that the center of the beam ran parallel to the table and the central fiber as seen in figure 2.3 with a reference scintillating fiber running next to it. The reference fiber was used to subtract any extra signal obtained in the protruding part of the central pixel; so, the left-over signal was equivalent to the signal from the length of the fiber embedded in the lead as illustrated in figure 2.4 [29]. A slight difference in readout between the simulation and the experiment occurs when obtaining the signal from the fibers between the two methods. In the case of the experiment the signal was recorded as charge in 
nC. While the amount of signal recorded for each event varies by $\mathrm{K} \sqrt{N}$ where $\mathrm{K}$ is the amount of deviation from the Poisson Distribution called the Fano-factor [15] and $\mathrm{N}$ is the amount of charges produced in an event. The average signal per event was obtainable by using many events per simulation. Since the average charge was created due the average number of optical photons interacting with the photomultiplier, the total signal was approximated by counting the number of optical photons exiting the detector at the far end. The discriminator threshold for the electrometer was not known while creating the simulation however since the signals were weighted by the number of optical photons reaching the photomultiplier tube (PMT), signals too weak to be detected by the electrometer would not contribute significantly to the total signal.

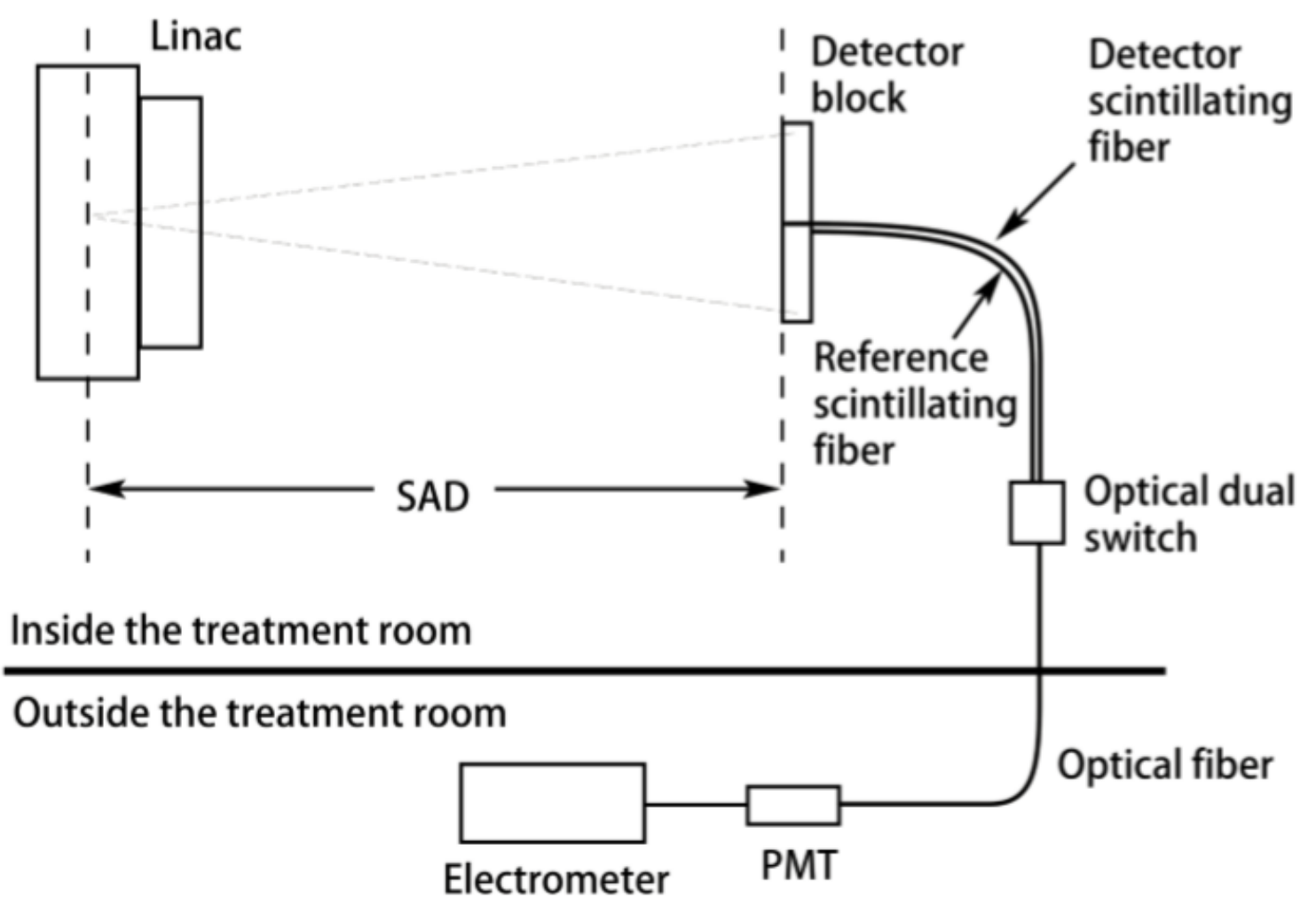

Figure 2.3 General Experimental setup. The signal was measured using two scintillating fibers. One embedded in the center of the detector and one to subtract additional signal generated in the length of the fiber protruding from the detector [34]. 


\section{optical fiber in detector block \\ optical fiber pulled back}

\section{net result}

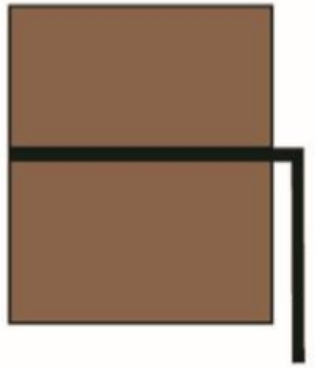

a

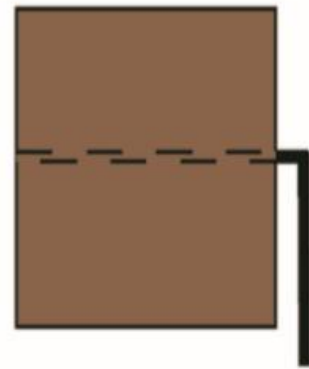

b
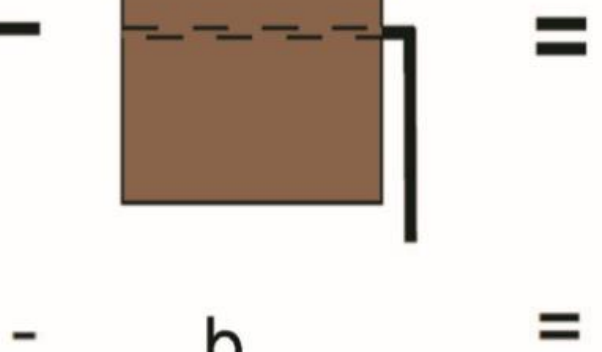

Figure 2.4 An illustration of the method of to obtain the signal in the embedded portion of the scintillating fiber. The example displays the method for optical fibers however the method is valid for scintillating fibers [29].

\subsection{Basic Physics Verification and Physics List Selection}

To perform the Monte Carlo simulation, a newly installed version of Geant4 10.03.p2 was used on a new installation of Ubuntu Linux. A sample simulation for the prototype detector written by a summer research student was provided as a starting point for the simulation match. The sample simulation was checked with a basic physics test to see if the output was as expected. This was done to verify that the code was working properly along with the installations. This check would streamline the code verification, minimizing the amount of the code that needed to be checked. Verification of the integrity of the provided code was done by comparing the attenuation coefficients of $x$-rays and the stopping power coefficients of electrons for mono energetic sources to the National Institute of Standards and Technology (NIST) database. This test served both as an integrity check and as a method to compare the available electromagnetic (EM) physics list packages to find the one that best fits the application. In Geant4 there are several prebuilt and verified physics lists available for the characterization of electromagnetic particle interactions. For most physics lists photon attenuation involves $x$-rays in the range of 10 $\mathrm{keV}$ and $1 \mathrm{GeV}$ and stopping power in a similar range. The lists of interest are emstandard_opt3, which is 
designed for higher accuracy of electrons, hadrons and ion tracking without magnetic field; emstandard_opt4, which is a higher accuracy model of emstandard_opt3; emlivermore, which is a modified version of emstandard_opt4; and empenelope, which is another modification of emstandard_opt4. Since all four of these physics lists are similar, it was necessary to test which one is best for the application at hand. To test these physics lists for attenuation accuracy a simulation was built using $300,0.1 \mathrm{~cm}$ thick slabs of water. A pencil beam was shone at normal incident to the slabs and the number of interactions in each slab were counted and then plotted and fitted with equation 2.1.

$$
N(x)=N_{0} e^{-\mu x}
$$

where $\mathrm{N}(\mathrm{x})$ is the total number of counts in slab $\mathrm{x}, \mathrm{N}_{0}$ is the incident number of $\mathrm{x}$-rays and $\mu$ is the total attenuation coefficient. This test was performed for monoenergetic $\mathrm{x}$-rays in the range of $10 \mathrm{keV}$ to $6 \mathrm{MeV}$ and compared against NIST photon cross section database (XCOM) attenuation values for water.

A similar test was also performed for stopping power with electrons however the metric that is to be compared to in NIST is the continuous slowing down approximation (CSDA) which is calculated using equation 2.2 [30]. The CSDA range is the average path length of an electron in matter [11]. To simulate CSDA range, mono-energetic electrons were fired from the center of a $1 \mathrm{~m}^{3}$ block of water. For every step length in the material the path length was recorded and summed. The average path length of the electrons was compared to the CSDA ranges from the NIST stopping power and range tables for electrons (E-STAR). The comparison between CSDA ranges was made for mono-energetic electrons ranging from 10 $\mathrm{keV}$ to $6 \mathrm{MeV}$ for the four different physics lists being considered.

$$
\text { Range }_{C S D A}=\int_{E 0}^{E f} \frac{d E}{S_{t o t}(E)}
$$

Equation 2.2

where $\mathrm{E}$ is energy, $\mathrm{Ef}$ is the final energy, $\mathrm{EO}$ is the initial energy and $\mathrm{S}_{\text {tot }}$ is the total stopping power [11]. 


\subsection{Simulation Validation Against Publicized Simulation}

Simulation validation was performed to benchmark the Geant4 simulation to a similar simulation performed and validated by a well known researcher in the field of portal imaging. This was done to show that the Geant4 simulation is accurate for applications similar to the experimental environment. Furthermore, this validation would support the accuracy of the data collected for the experimental validation. The simulation was validated against the simulation outlined in the paper by D A Jaffray et al in 1994 which used Electron Gamma Shower 4 (EGS4) [31].

The simulation described in Jaffray's paper indicates that: a cone beam was used, with its field size set at $100 \mathrm{~cm}$ from the point source that is the tip of the cone; a slab of PMMA (the material used was described previously), with its farthest face from the point source placed at $100 \mathrm{~cm}$ from the tip of the cone beam; and a radial scoring surface with a diameter of $5 \mathrm{~cm}$ [31]. In the Geant4 Simulation setup a uniform cone beam was setup by first setting the beam start point to $(0,0,0)$ in the world volume defined, using a random number generator that ranges from 0 to 1 (not including 0 ) and taking the square root of this (equation 2.3) the radius can be varied so that if a circular beam is swept out, the intensity of the beam will be uniform throughout the area.

$$
\text { radius }=\text { fieldsize } * \operatorname{sqrt}(\text { G4UniformRand }(\quad))
$$

Equation 2.3

Field size is the maximum radius of the field to be swept out by the beam, G4UniformRand() is a predefined function in Geant4 that generates a number from 0 to 1 (not including 0 ).

The phantom described in Jaffray's paper was set as a slab of infinite length and width with varying thickness. To match this the phantom was set with a length and width of $2 \mathrm{~m}$ to make it infinite in size relative to the maximum field size of the cone beam which was to be varied up to a diameter of $30 \mathrm{~cm}$ at the far size of the phantom. The scoring area was defined using the G4tubs class (used for cylinders) as a thin disk of radius $2.5 \mathrm{~cm}$ made of air and placed at some distance from the phantom along the central axis of the beam path. The distance described will be referred to as the air gap from herein to conform with the terminology in Jaffray's paper [31]. 
The scoring was handled in Jaffray's paper by assigning a weighting of $1 / \cos \theta$ to each $x$-ray based on their incident angle to the scoring surface where an angle of $0^{\circ}$ is normal to the scoring surface. To obtain the same thing, the positions of the x-rays were determined before and after entering the scoring surface and Pythagoras's equations were used to determine the lengths of the adjacent and hypotenuse sides of the triangle created by its motion rather than calculating the angle directly (equations 2.4a, 2.4b and 2.4c). To prevent the weighting factor from reaching infinity the maximum angle allowed was 85 degrees [31].

$$
\begin{gathered}
\frac{1}{\operatorname{Cos} \theta}=\frac{h y p}{a d j} \\
h y p=\sqrt{\left(x_{i}-x_{f}\right)^{2}+\left(y_{i}-y_{f}\right)^{2}+\left(z_{i}-z_{f}\right)^{2}} \\
a d j=\left|z_{i}-z_{f}\right|
\end{gathered}
$$

Equation $2.4 c$

In Jaffray's simulations, restrictions on the creation of secondary particles and the transport of all particles was limited to values of $10 \mathrm{keV}$ for photons and $711 \mathrm{keV}$ for electrons to reduce the amount of simulation time required [31]. Even though the simulation time for this sort of setup is not a problem today, to keep the replication consistent these restrictions were mimicked none-the-less. A couple of differences arise here between EGS4 and Geant4, the first significant difference between the two simulation packages is in defining the creation threshold. In EGS4 an energy value can be set directly for the creation threshold but in Geant4 this value is defined in terms of how far the particle must be able to travel in the material which is later converted into energy which is defined using the prebuilt variable called cut length. The cut length was set to $24.7 \mathrm{~mm}$ for gamma cut (GCUT) and $2.34 \mathrm{~mm}$ for electron cut (ECUT), these were calibrated by monitoring the threshold values listed when the simulation is in the initialization phase, the values were tweaked until the desired threshold values were obtained. The second significant difference comes from trying to set transportation thresholds. By default, the transportation energy cut for Geant4 is around $1 \mathrm{eV}$, to define this in Geant4 thresholds were set in the 
user defined SteppingAction class in the program. Since it was not directly specified in Jaffray's paper [31] or in the EGS4 manual [32] that the $711 \mathrm{keV}$ threshold was for both electrons and positrons, the cuts and transport thresholds for positrons were left at their default values.

From Jaffray's paper [31] figures 4, 5 and 8 were matched using the same parameters specified in the paper and described in the methods section. For figures 4 and 5 mono-energetic beams were used while for figure 8 a polyenergetic beam had to be used to obtain the match. For measurements that required a poly-energetic source, the spectrum from a paper by Kubsad et al [33] was used. Figure 2.5 shows the spectrum used for a $6 \mathrm{MV}$ source which, for the purposes of the project, was the only energy source considered.

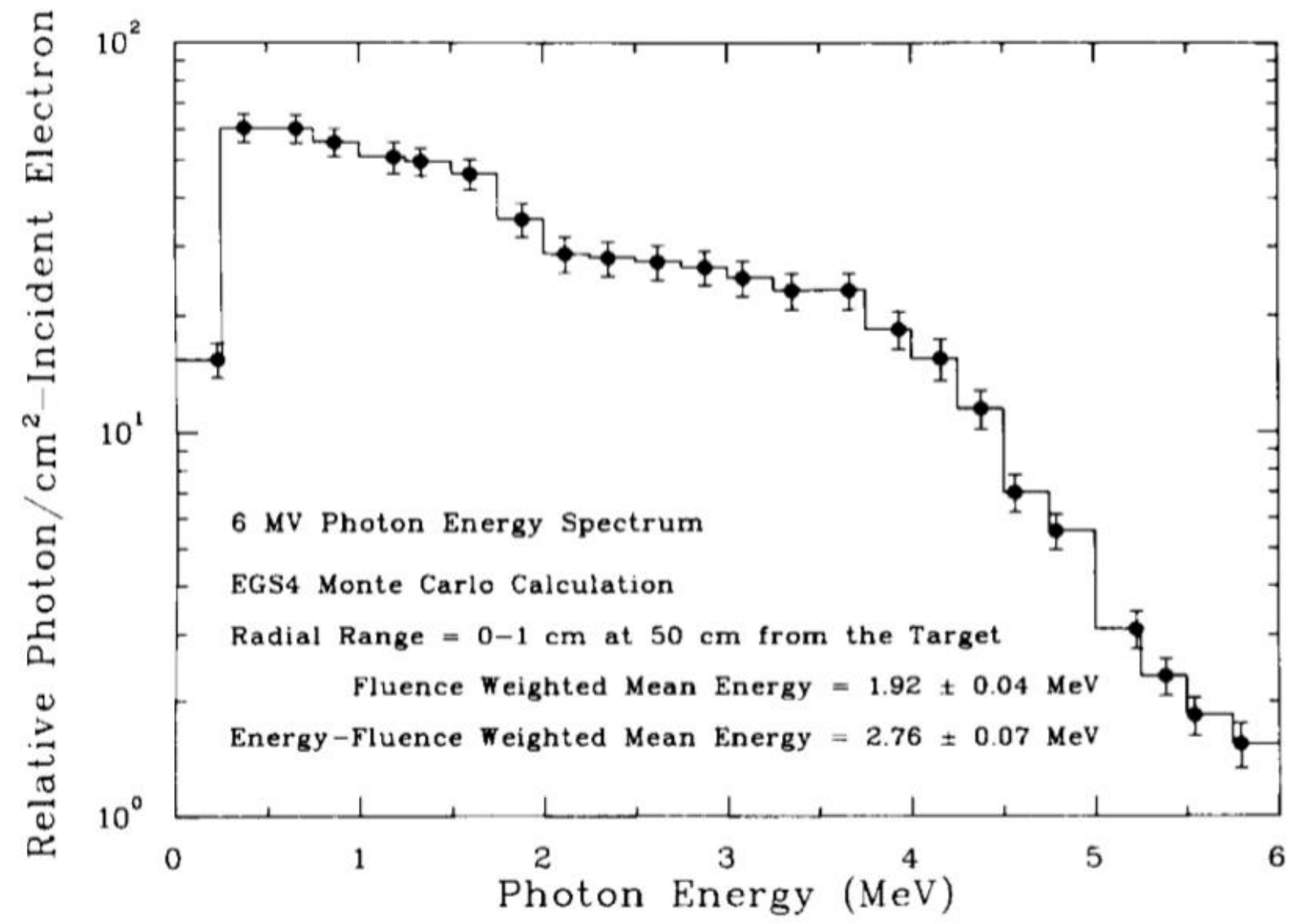

Figure 2.5 The polyenergetic spectrum used for the simulation validation [33]. 


\subsection{Simulation Validation Against Experimental Results}

Experimental validation was performed with the transport and creation threshold for all particles set at their default values. Comparisons were made between the simulation and experiment for the spatial resolution through the MTF and the SPR through the total signal received as the field size varied from $3 \mathrm{~cm}$ by $3 \mathrm{~cm}$ to $20 \mathrm{~cm}$ by $20 \mathrm{~cm}$. The spectrum used for the simulation shown in figure 2.6 is for the Pinnacle3 treatment planning system (TPS) [34]. Some discrepancy is expected between the spectrum shown in figure 2.6 and the actual spectrum used in the experiment.

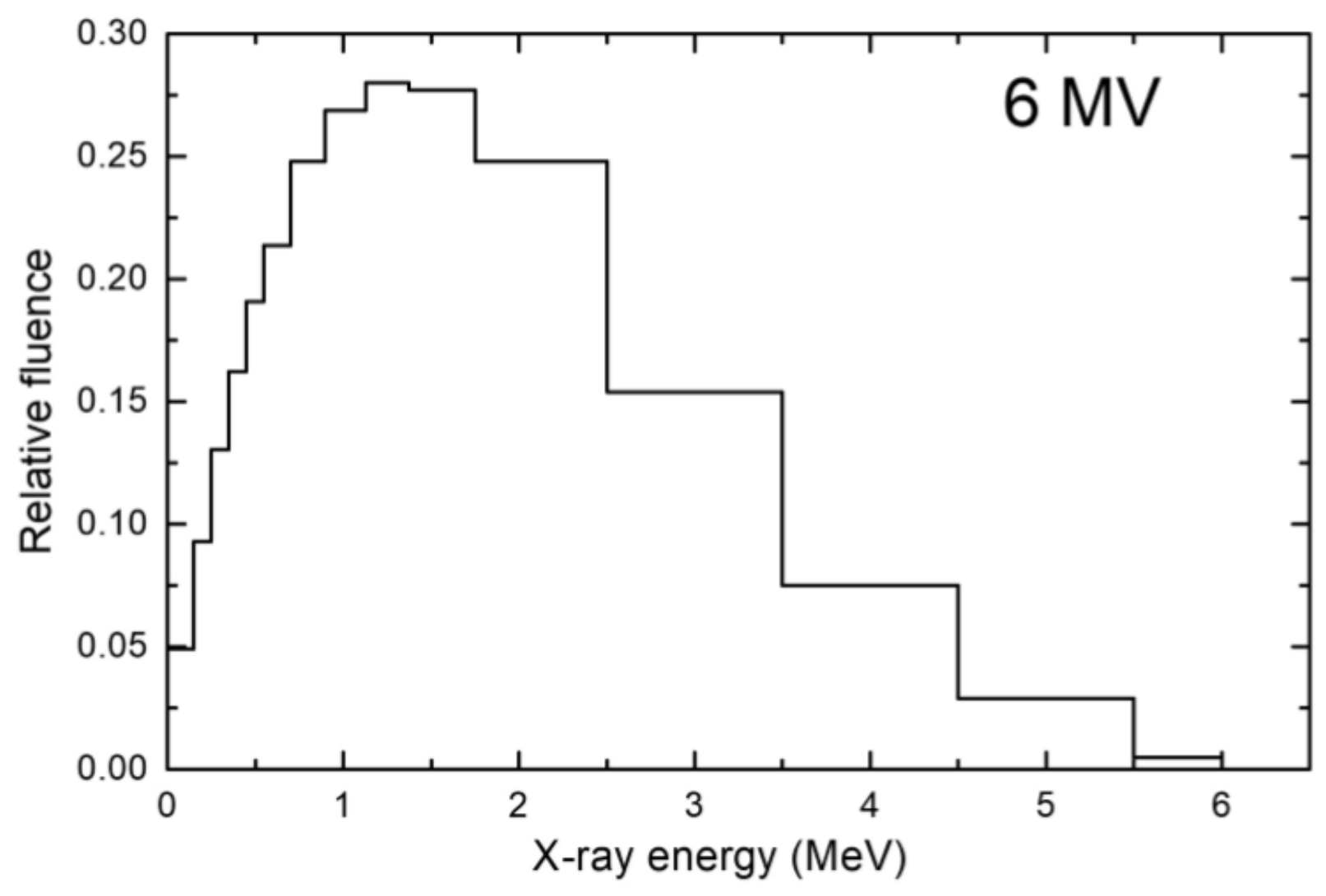

Figure 2.6 The poly energetic spectrum used in the simulation to compare to the experimental results for the prototype detector. This spectrum is from the Pinnacle 3 treatment planning system [34]. 


\subsubsection{Experimental method for Spatial resolution}

Spatial resolution was experimentally measured [34] using the slit assembly composed of two 3.5 $\mathrm{cm}$ by $7 \mathrm{~cm}$ by $10.5 \mathrm{~cm}$ steel blocks shown in figure 2.7 spaced apart with shims 80 um thick. The detector was mounted on a translation stage and was used to measure the signal intensity in the central fiber for a range of positions. The LSF was recorded by shifting the stage from left to right and recording the signal strength at each location. Figure 2.8 shows the measured LSFs for along both the $x$-axis and the $y$-axis [34]. All experimental measurements were performed by Jian Liu [34] however a detailed understanding of the methods used in the experiment was useful in determining how the simulation should be performed.

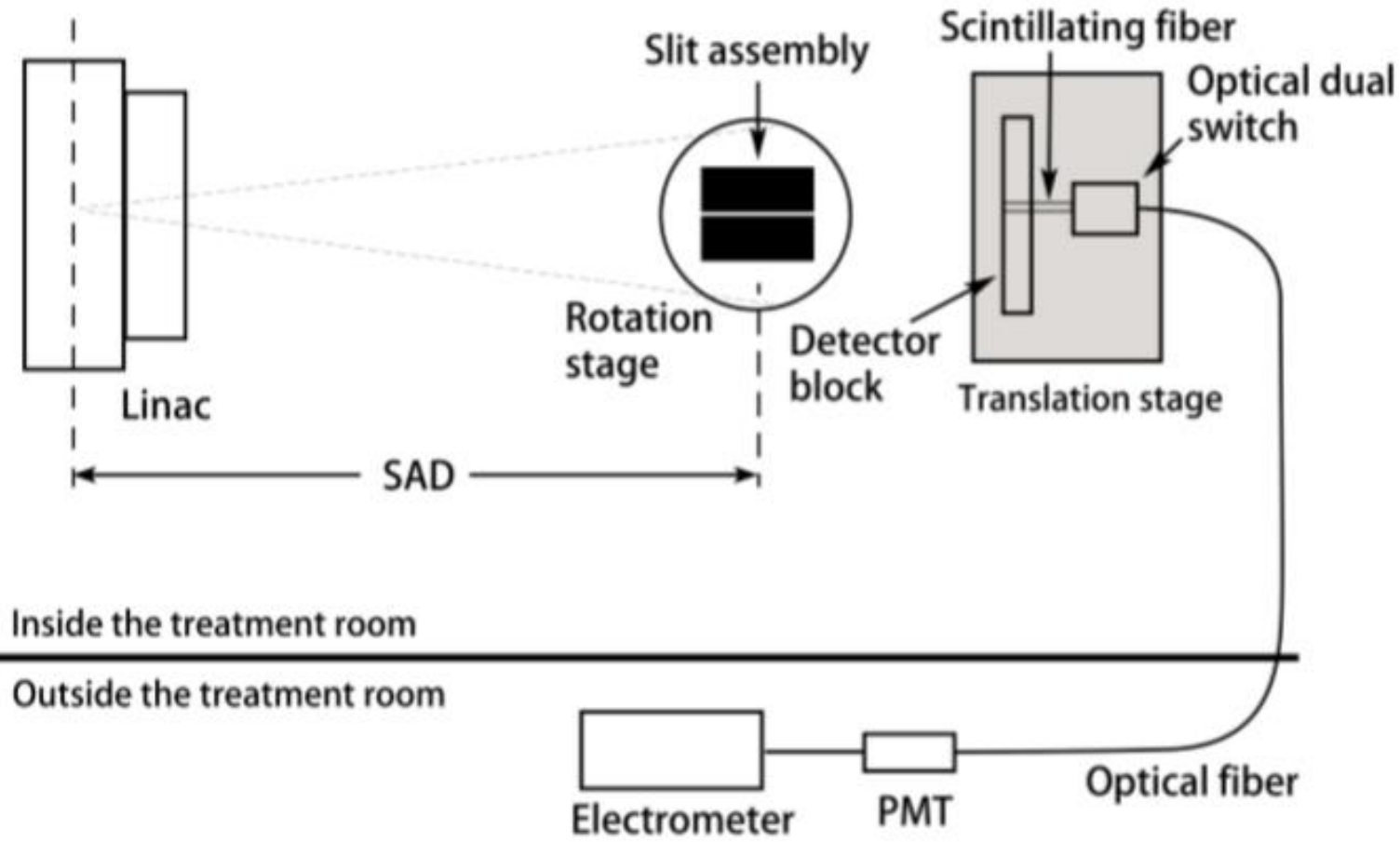

Figure 2.7 The experimental setup for the spatial resolution measurements [34]. 


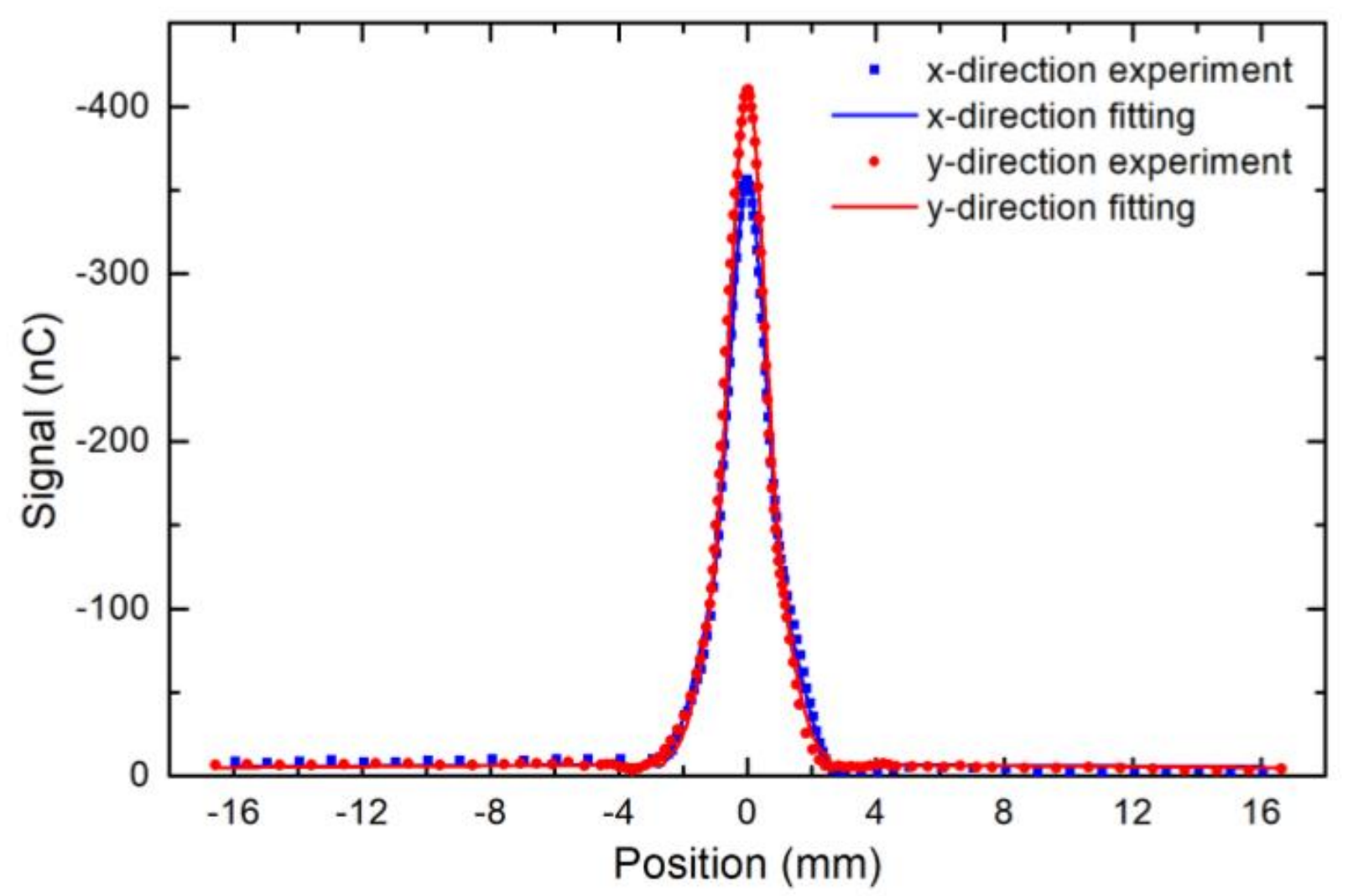

Figure 2.8 The experimentally measured line spread functions for both the $x$-axis and the $y$-axis [34].

\subsubsection{Simulation Method for Spatial Resolution}

The simulation had the benefit of having every fiber in the detector active which reduced the need to scan the beam across a significant portion of the detector. In addition, a pencil beam was used instead of using a slit assembly and the LSF function found using equation 2.5 from the PSF. To reduce the differences between the experiment and the simulation an 80um wide line source with a height of one pixel was used. Since the beam width was smaller than the width of one pixel, $1.35 \mathrm{~mm}$, the pencil beam was not shift invariant within the pixel itself. To account for this the pencil beam was scanned left to right from the center of one pixel to the next. The LSF was reconstructed using the methods described by Fujita et al [35]. The LSF was measured for 36 different pencil beam positions between the central column and the right adjacent column in as many separate simulations. This method is used to measure the presampling MTF through a Fourier transform for a finely sampled LSF [35]. 


$$
\operatorname{LSF}(i)=\sum_{j=0}^{j=N} P S F(i, j)
$$

Conversion from the presampling MTF into the observable MTF was done by convolving the presampling MTF with the aperture function (equation 2.6) and the sampling comb function (equation 2.7) to account for aliasing effects due to under sampling $[13,26]$.

$$
\begin{gathered}
\text { Aperturefn }=\frac{\sin (f \pi h)}{f \pi} \\
\text { Combfn }=\frac{1}{h}
\end{gathered}
$$

where $\mathrm{f}$ is sample frequency which is determined by equation 2.8 and $\mathrm{h}$ is the width of the pixel.

$$
f=\frac{i}{\text { DetWidth }} ; i \in N ;\left(0 \leq i \leq \frac{\text { NumSamples }}{2}\right)
$$

where ' $\mathrm{i}$ ' is a natural integer ranging from 0 to half of the number of data points that make up the LSF being transformed.

\subsubsection{Experimental Method for Scatter Primary Ratio}

The scatter primary ratio was measured [34] to determine the detectors performance in a larger area beam. The experimental setup, shown in figure 2.9, consisted of a solid water phantom with dimensions of $30 \mathrm{~cm}$ by $30 \mathrm{~cm}$ by $30 \mathrm{~cm}$ placed at the LINACs isocenter at $100 \mathrm{~cm}$ from the beam source. The detector was placed a distance from the phantom, creating an air gap, with the central pixel placed centered with the LINAC beam. The total signal was measured in the central fiber for a series of field sizes ranging from $3 \mathrm{~cm}$ by $3 \mathrm{~cm}$ to $30 \mathrm{~cm}$ by $30 \mathrm{~cm}$ for a series of five different air gaps ranging from $5 \mathrm{~cm}$ to $75 \mathrm{~cm}$ producing the result shown in figure 2.10. All experimental measurements were performed by Jian Liu [34] however a detailed understanding of the methods used in the experiment was useful in determining how the simulation should be performed. 


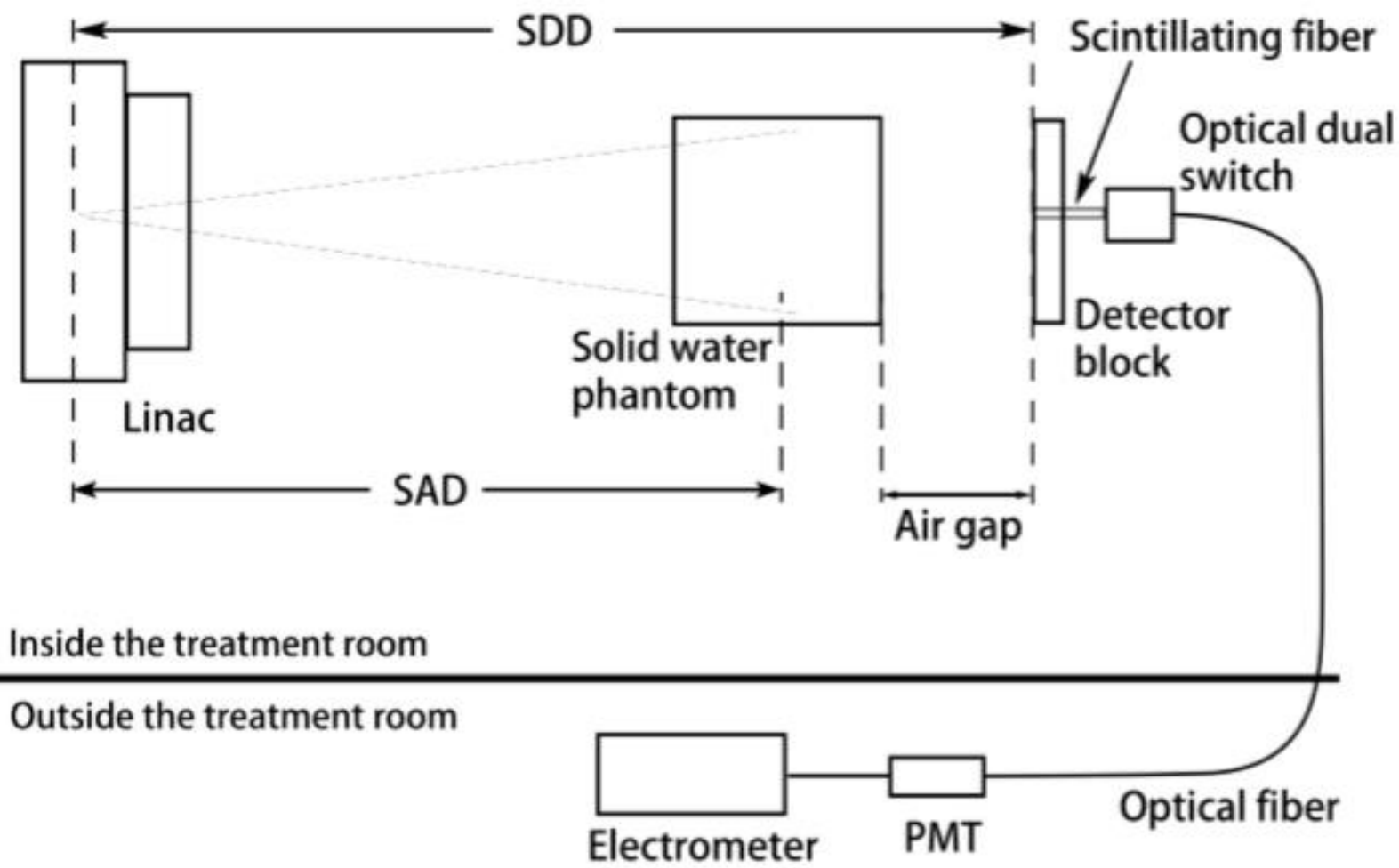

Figure 2.9 The Experimental setup for the scatter primary ratio versus field size measurements [34]. 


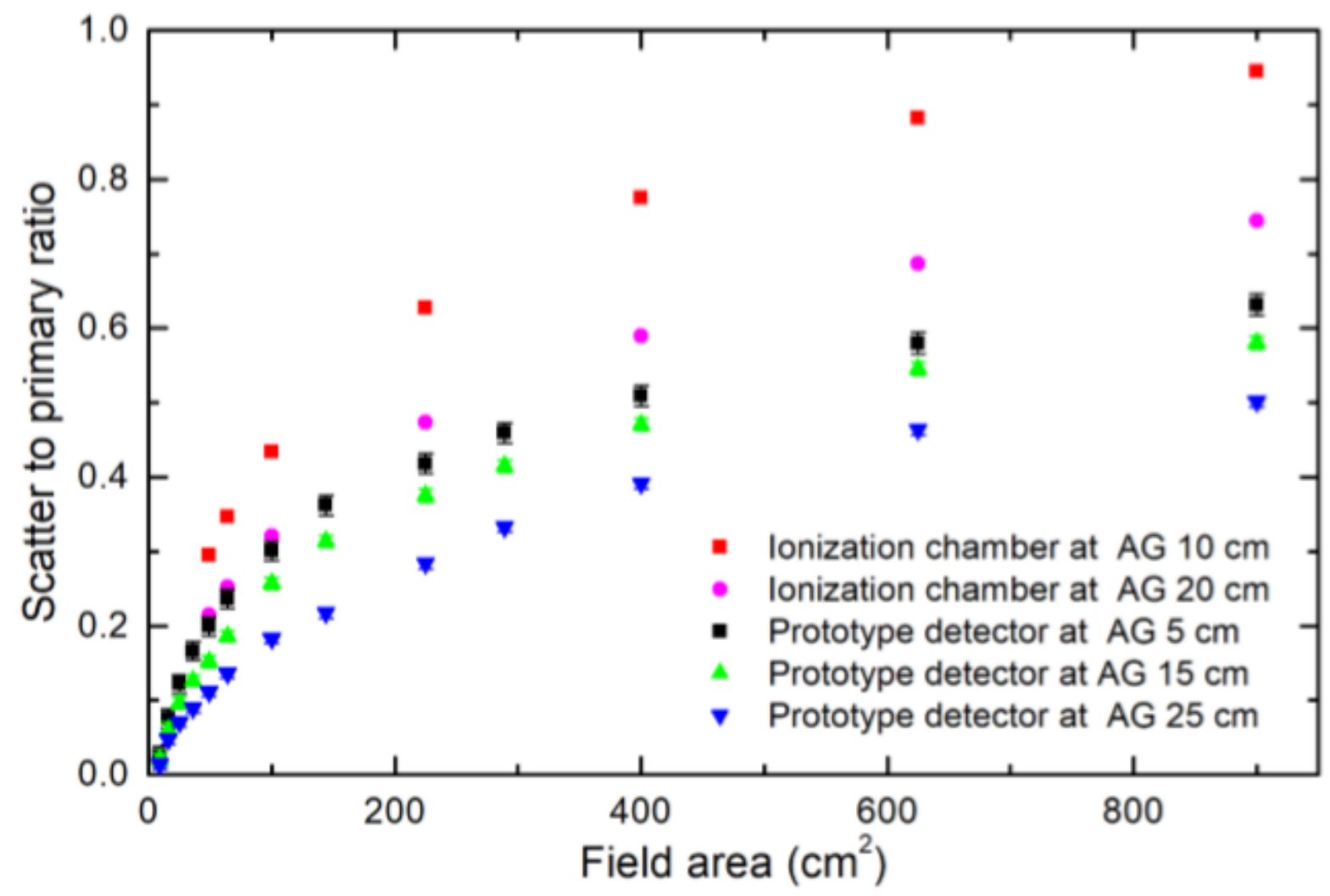

Figure 2.10 The experimental results for the scatter primary ratio measurements [34].

Since the signal from primary $\mathrm{x}$-rays and the signal from scattered $\mathrm{x}$-rays are generally indistinguishable from each other in the experimental setting, the primary signal was instead inferred as the total signal that would be measured for a field size of $0 \mathrm{~cm}$ by $0 \mathrm{~cm}$. To obtain the signal from a $0 \mathrm{~cm}$ by $0 \mathrm{~cm}$ cone beam the total signal was plotted as shown in figure 2.11 for the various field sizes down to the smallest available field size of $3 \mathrm{~cm}$ by $3 \mathrm{~cm}$ and then fitted with a spline from Matlab to extrapolate the signal to $0 \mathrm{~cm}$ by $0 \mathrm{~cm}$. The SPR was then calculated using equation 2.9 where $P$ is the total signal at a field size of $0 \mathrm{~cm}$ by $0 \mathrm{~cm}$ [34].

$$
S P R=\frac{T-P}{P}=\frac{S}{P}
$$

However, since the equation of the fitting spline was unknown it was decided to use the total signal instead. Logically if the total signals are comparable for the different field sizes and airgaps then the SPR must also be comparable regardless of the primary signal used in each measurement. The total signal was obtained from the measured results using the SPR values. Since each SPR value is the fraction 
of scatter signal relative to the primary signal, the total signal could be obtained by adding 1 to the SPR as shown in equation 2.10 since the primary signal $P$ in this ratio is 1 . The total signal was then scaled such that the total signals matched for a field size of $10 \mathrm{~cm}$ by $10 \mathrm{~cm}$. The total signals were matched for this field size because the collimator scatter factor is calibrated to be 1 for this field size for every machine.

$$
T=\frac{S}{P}+P ; \text { if } P=1
$$

Equation 2.10

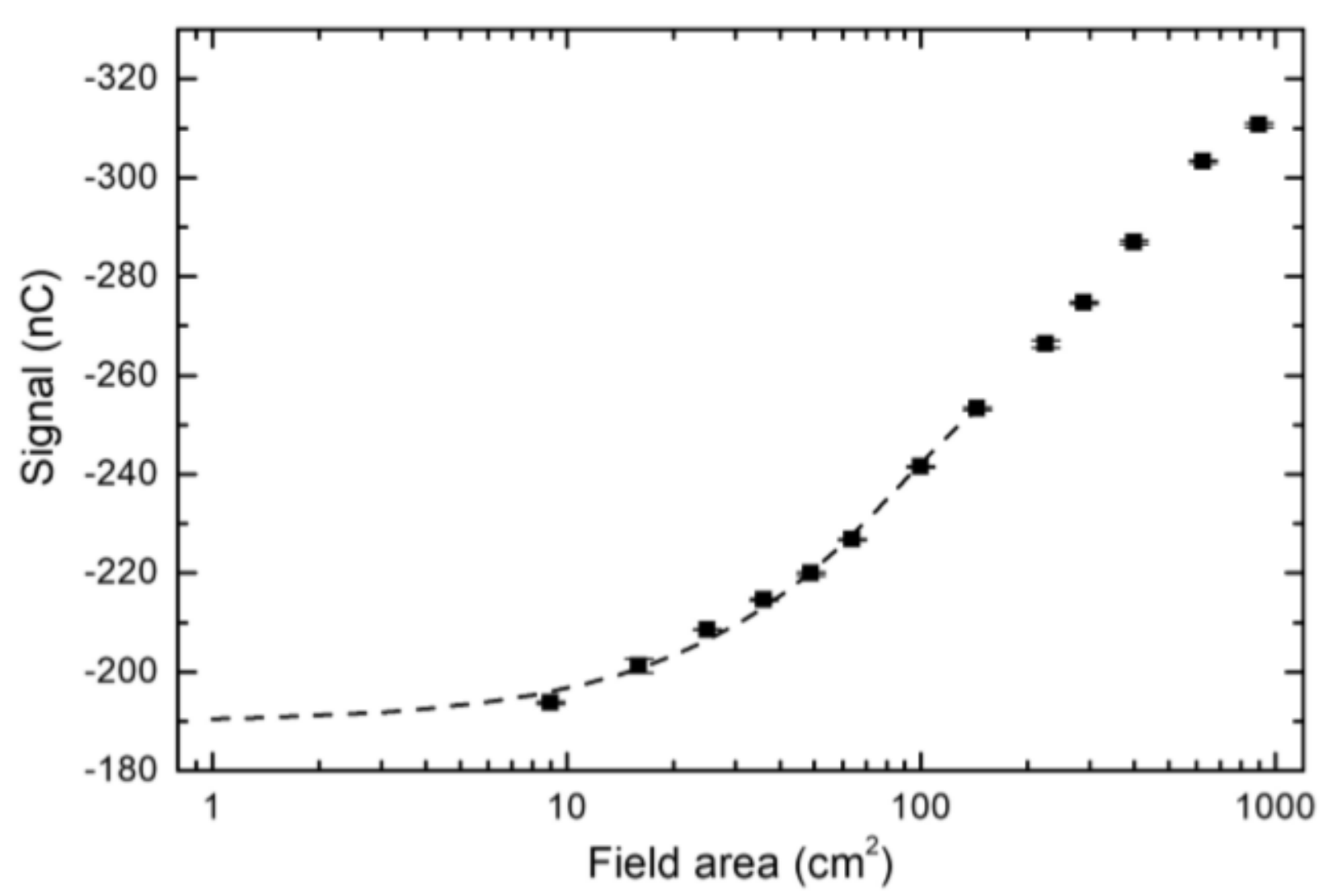

Figure 2.11 The method to determine the primary signal for the experimental measurements. The primary signal for each field size was determined by an extrapolation to zero field size [34]. The extrapolation function was unknown during the simulations. 


\subsubsection{Simulation Method for Scatter Primary Ratio}

The scatter primary ratio in the simulation was measured using a pixel sized pencil beam centered on the central pixel of the detector. As demonstrated in figure 2.3 the experiment measured the signal produced in a single fiber from a cone beam of some field size. Producing a similar simulation was not practical due to time restrictions on the project. Instead a pixel sized pencil beam incident on the central pixel was used. The pixel size chosen was $1.35 \mathrm{~mm}$ by $2.44 \mathrm{~mm}$ which would cover a central fiber completely while covering a quarter of each of the nearest neighbors creating a shift invariant pixel.

Considering the total signal is measured with a single pixel in the experiment for a large dose, spatial invariance was available to be used in respect the average signal distribution in accordance to the methods by Teymurazyan et al [36]. The paper describes the discrete signal distribution shown in equation 2.11 a and $2.11 b$.

$$
\begin{gathered}
\bar{R}_{P}(j, k)=\sum_{i^{\prime}, j^{\prime}} \bar{K}_{P}\left(j, k ; j^{\prime}, k^{\prime}\right) \times \varphi\left(j^{\prime}, k^{\prime}\right) \times \alpha\left(j^{\prime}, k^{\prime}\right) \\
\bar{R}_{S}(j, k)=\sum_{i^{\prime}, j^{\prime}} \bar{K}_{S}\left(j, k ; j^{\prime}, k^{\prime}\right) \times \varphi\left(j^{\prime}, k^{\prime}\right)
\end{gathered}
$$

where $\mathrm{Rp}+\mathrm{Rs}$ is the total signal received at fiber $\mathrm{j}, \mathrm{k}$ from the all incident $\mathrm{x}$-rays with fluence $\varphi$ and attenuation $\alpha$ (for primary $\mathrm{x}$-rays) generating an average number of optical photons $\mathrm{K}$ in some distant fiber $j^{\prime}, k^{\prime}$.

If instead the summation sign is removed from both equations and only a single pencil beam incident on fiber $j^{\prime}, k^{\prime}$ is considered then the amount of signal contributed to fiber $j, k$ becomes well defined at some value for a large number of incident $\mathrm{x}$-rays. The signal received at $\mathrm{j}, \mathrm{k}$ is indistinguishable from the signal received at $j^{\prime}, k^{\prime}$ if the roles of $j, k$ and $j^{\prime}, k^{\prime}$ are reversed and instead the pencil beam is incident on pixel $\mathrm{j}, \mathrm{k}$. This means that the amount of signal received in a distant fiber is only dependent on the distance making the signal shift invariant provided the distance between pixels are the same. Considering that the experiment is performed with only a single active fiber and a large field size while the simulation is performed with all of the fibers active and a pixel sized pencil beam the two approached are approximately the same considering the shift invariance of the signal. The cone beam can be considered as a series of pencil beams each contributing an amount of signal to the central fiber in accordance to equations $18 \mathrm{a}$ and $18 \mathrm{~b}$. While the simulation is a single pencil beam contributing an amount of signal to 
each of fibers in an opposing fashion to the experiment. If in the simulation each of the active fibers is considered to be the central pixel shifted over by some distance the equations above can be used in a similar fashion as in the experiment. The equations $2.11 \mathrm{a}$ and $2.11 \mathrm{~b}$ can be modified to express this as shown in equation $2.12 \mathrm{a}$ and $2.12 \mathrm{~b}$ by swapping the $\mathrm{j}^{\prime}$ and $\mathrm{k}^{\prime}$ with $\mathrm{j}$ and $\mathrm{k}$.

$$
\begin{gathered}
\bar{R}_{P}(j, k)=\sum_{i^{\prime}, j^{\prime}} \bar{K}_{P}\left(j^{\prime}, k^{\prime} ; j, k\right) \times \varphi(j, k) \times \alpha(j, k) \\
\bar{R}_{S}(j, k)=\sum_{i^{\prime}, j^{\prime}} \bar{K}_{S}\left(j^{\prime}, k^{\prime} ; j, k\right) \times \varphi(j, k)
\end{gathered}
$$

Here the total signal received in a fiber $\mathrm{j}, \mathrm{k}$ is determined by the sum of the signals recorded in fibers $j^{\prime}, k^{\prime}$ from a beam incident on pixel $j, k$. If only a single fiber is being considered in the equations $2.11 \mathrm{a}$ and $2.11 \mathrm{~b}$ then equations $2.12 \mathrm{a}$ and $2.12 \mathrm{~b}$ are equivalent. This allows the total signal in the central fiber in the simulation to be built up by adding up the signals in the surrounding fibers and an area beam to be reverse engineered by adding up the total signal contribution in an equivalent area as shown in figure 2.12 .

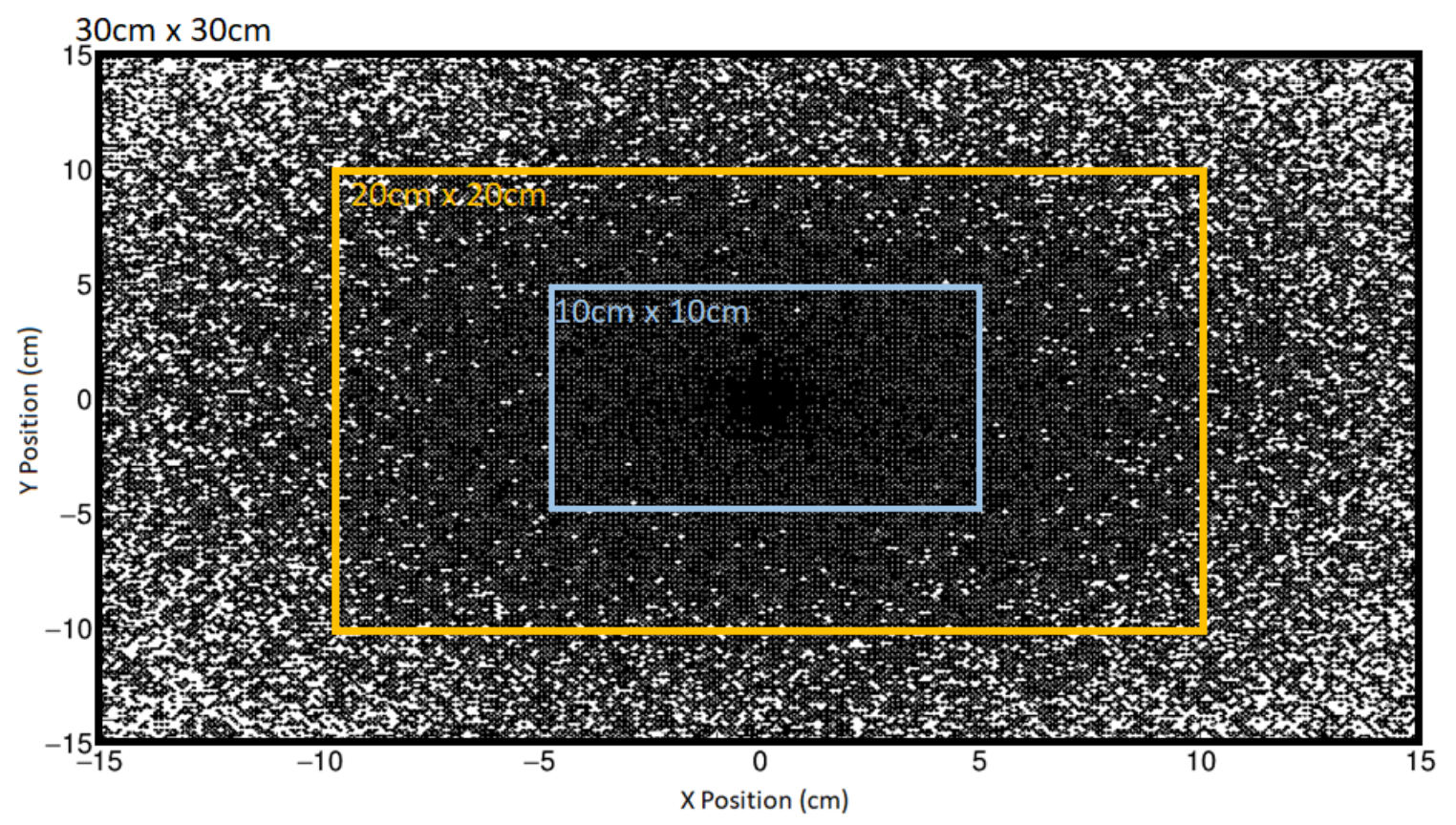

Figure 2.12 An example of how the total signal for various field sizes were determined. The signal for each of the pixels inside the areas were added up for each of the field sizes. The field sizes shown are the equivalent field sizes for a cone beam at the isocenter. 


\subsubsection{Determining the Equivalent Field Size}

The signal received in a central fiber for both experiment and simulation depended on the field size of each of the beams. However, the definitions of the two field sizes differ since in the experiment the field size is measured at the isocenter and in the simulation the field size is ultimately determined at the face of the detector as shown in figure 2.13.

There are three main sources of scattering in the experiment: the first source is from the LINAC head and the flattening filter, which is designed to create a flat dose profile at a depth of $10 \mathrm{~cm}$ in water; the second source is the phantom, where the beam transverses before reaching the detector; and the final source of scatter is the detector itself. The scatter from the LINAC head can seen as the same for both sources if the additional dose contributions for the various field sizes are considered for whichever approach is used. The detector scatter is similar between the two beams, with the exception of some skew from an angled pencil beam as opposed to one that is of normal incidence, both the pencil beam from the simulation and the angled pencil beams from the experiment produced signal distributions with similar widths as measured through the standard deviation. The distribution of the signal had a range of about $5 \mathrm{~mm}$ from detector scatter indicating that there is little contribution to the signal in the central fiber in the experiment and surrounding fibers in the simulation outside this range. The main source of the signal distribution from scatter was from the phantom.

Since the simulated beam is just a single pencil beam normal to the surface of the detector, the built up signal is the equivalent signal from a large area parallel beam as indicated in figure 2.13. The difference in angle means that the effective beam from the simulation and the cone beam in the experiment will sweep out different volumes of the phantom. Equating the two beams was done by making the volume of interaction with the phantom the same, figure 2.13 displays the approach used, while the cone beam sweeps out less volume in the phantom prior to the isocenter, this volume is compensated for after the isocenter where the cone beam sweeps out more volume than the parallel beam. While the additional volume swept out by the cone beam in the second half of the phantom is larger than the volume missed in the first half, contributions are the same when the two halves of the cone beam are weighted by intensity. In fact, the average intensity of the parallel beam and the cone beam are equivalent if attenuation is ignored and the number of initial $x$-rays is the same. 


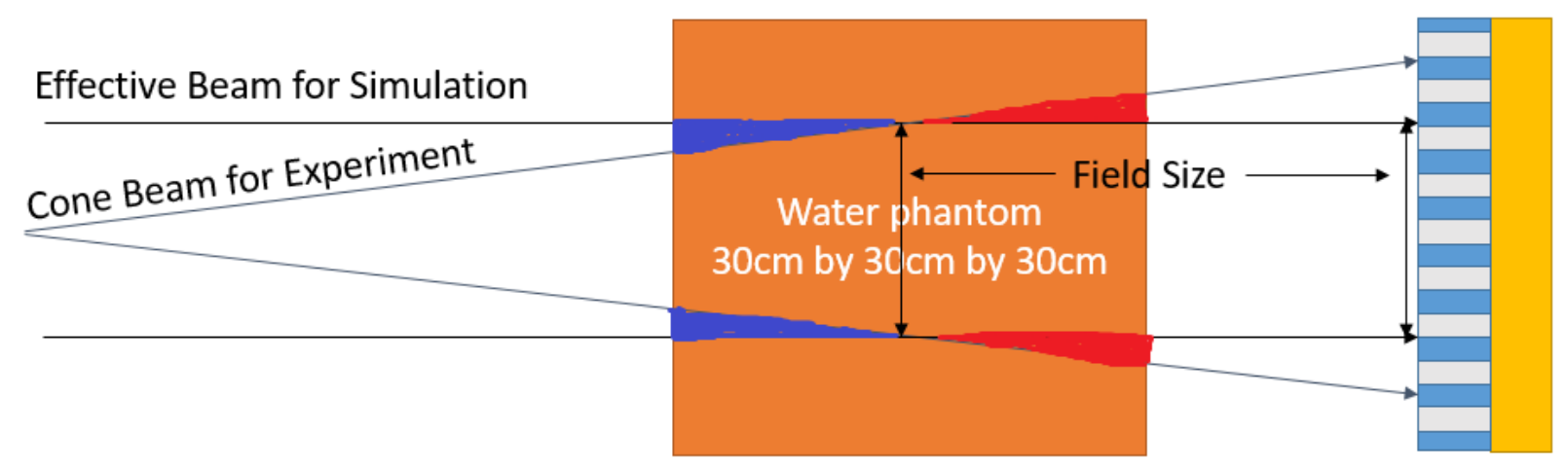

Figure 2.13 The illustrated comparison between the effective simulation beam and the actual beam for the experiment. The blue area is the area missed by the cone beam but included in the simulation beam, the red area shows the area for the opposite of this. The differences in volume is compensated by the intensities.

\subsection{Collimator scatter}

Collimator scatter could not be simulated instead the additional dose contributions from the amount of flattening filter visible was modified using the measured values shown in figure 2.14. For field sizes that fell between available values linear interpolation was used. The total signal was multiplied by the collimator scatter factor for the appropriate field sizes. 


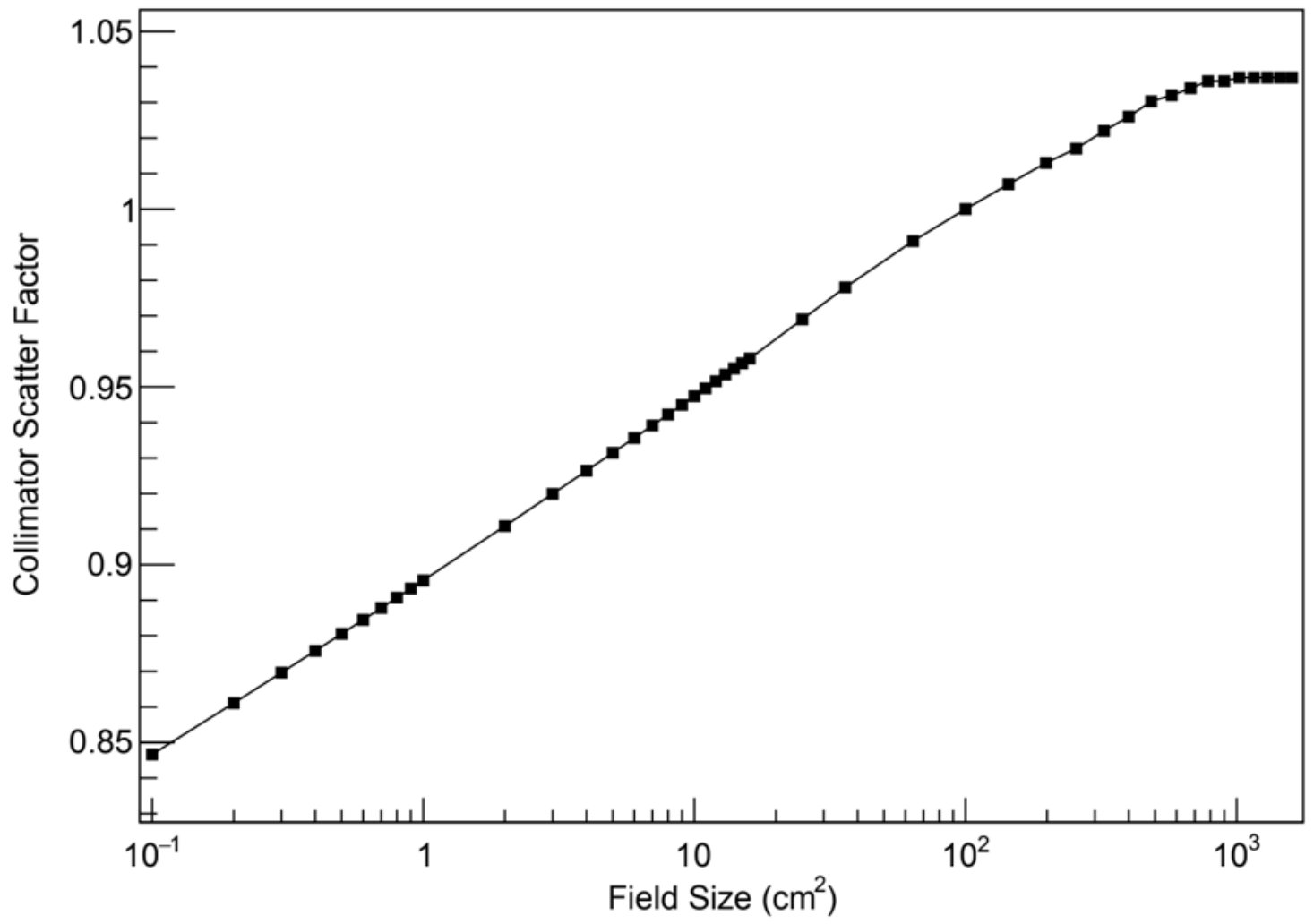

Figure 2.14 The collimator scatter factor curve for the machine used in the experiment. A semi log graph was used to clearly show the scatter factors for the smaller field sizes this is necessary since the smallest field size used in the simulation was approximately $3 \mathrm{~mm}^{2}$.

\subsection{Detector Design Optimization}

Optimization of the detector was attempted by changing the shape of the fibers to square and the array to a square array. Figure 2.15 shows the design the glue strips were moved to run from corner to corner of the fibers for every layer of lead since this is likely possible to build. The amount of glue used was set to a thickness of $85 \mathrm{um}$ adding to the distance between pixels, this is roughly a similar amount of glue used in the previous design. The goal with this new design is to optimize the spatial resolution of the detector while keeping it feasible to build. To keep the design feasible, the materials were required to be commercially available. 


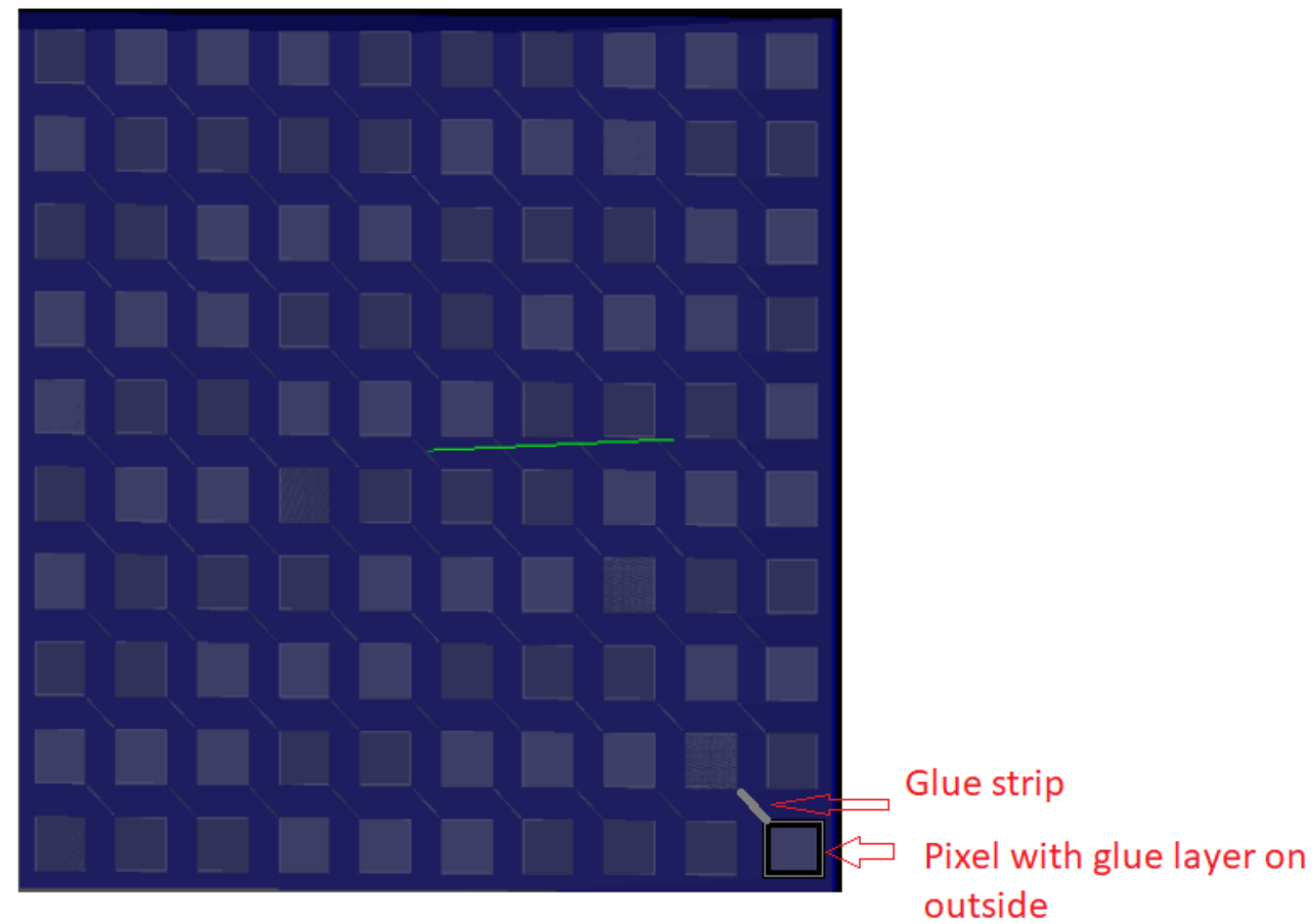

Figure 2.15 Close up of the new detector design for the optimization experiments. The glue was placed in the corner of the pixels since it was thought to be easier to build the detector this way.

Spatial resolution measurements were performed in the same way as for the previous detector design. A total of 36 simulations were performed with a 1 pixel tall, 80 um wide pencil beam that was shifted from central pixel to the next. The LSF was reconstructed using the method described by Fujita [35] and the observed MTF was obtained by convolving the presampled MTF with the aperture function and the comb function. 



\section{Chapter 3}

\section{Results}

\subsection{Basic Physics Verification and Physics List Selection}

The attenuation coefficients were checked using a segmented block of water. Each segment was $0.1 \mathrm{~cm}$ thick for a total of 300 segments. The results of the simulations were compared to the values found in the NIST XCOM database. From figure 3.1 the tests showed that for very low energies from $10 \mathrm{keV}$ to $50 \mathrm{keV}$ the most accurate physics list was option 4. Then from $50 \mathrm{keV}$ to $1 \mathrm{MeV}$ option 4, Livermore and Penelope performed similarly and finally for energies from $1 \mathrm{MeV}$ to $6 \mathrm{MeV}$ the Penelope physics list was the most similar to the NIST XCOM database. Since the average energy of the Pinnacle 3 TPS 6 MV spectrum is around $2 \mathrm{MeV}$ the best choice for these energies was the either option 4, Livermore or Penelope physics lists. Stopping power was measured using an infinite volume of water with monoenergetic electrons by measuring their CSDA range. The comparison between simulation and NIST E-STAR values can be seen in figure 3.2, Penelope and emoption4 showed consistently the most accurate results for energies from $100 \mathrm{keV}$ to $5 \mathrm{MeV}$. 


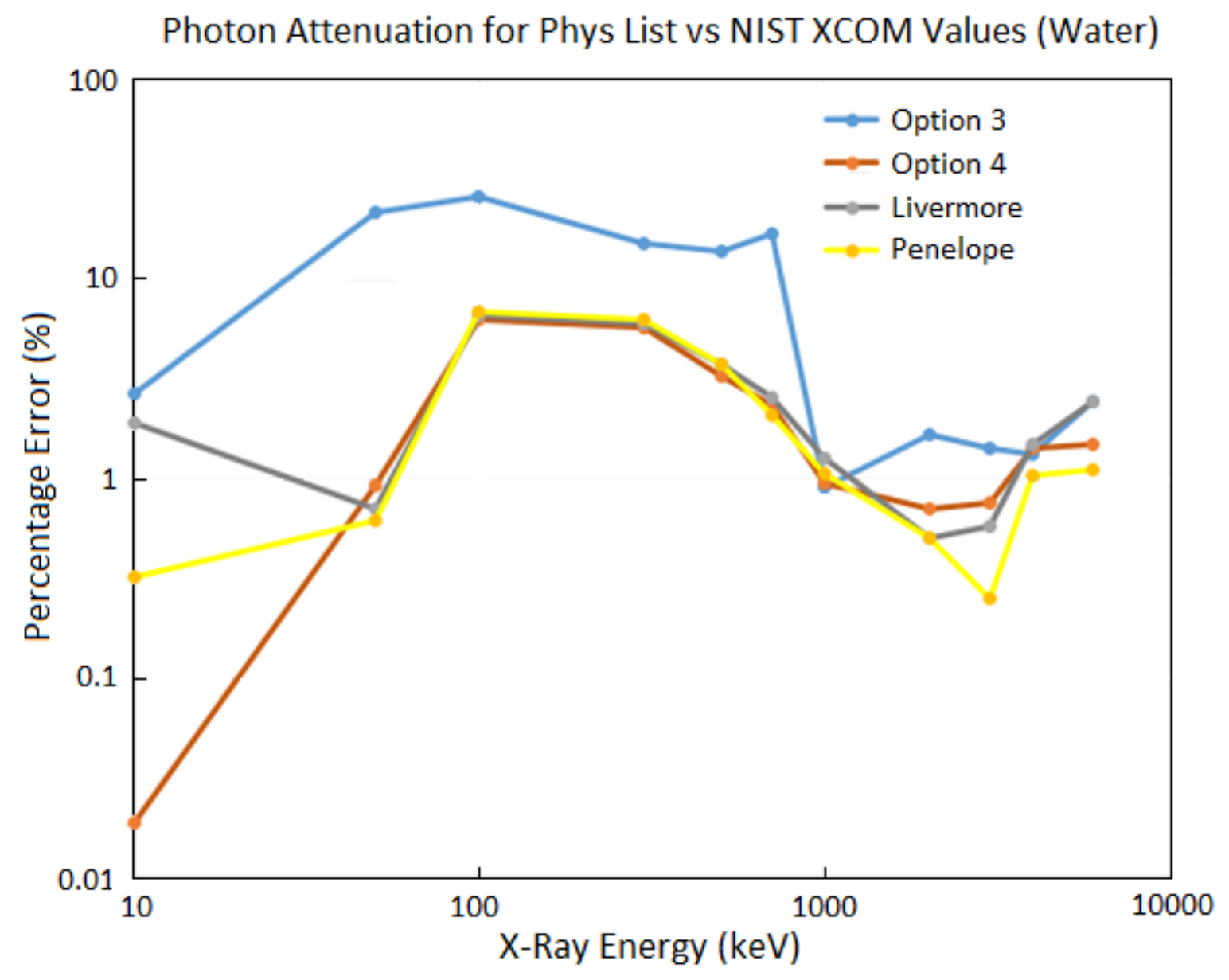

Figure 3.1 Prebuilt simulation code performance for attenuation. The simulation was performed using $3000.1 \mathrm{~cm}$ thick water slabs. 


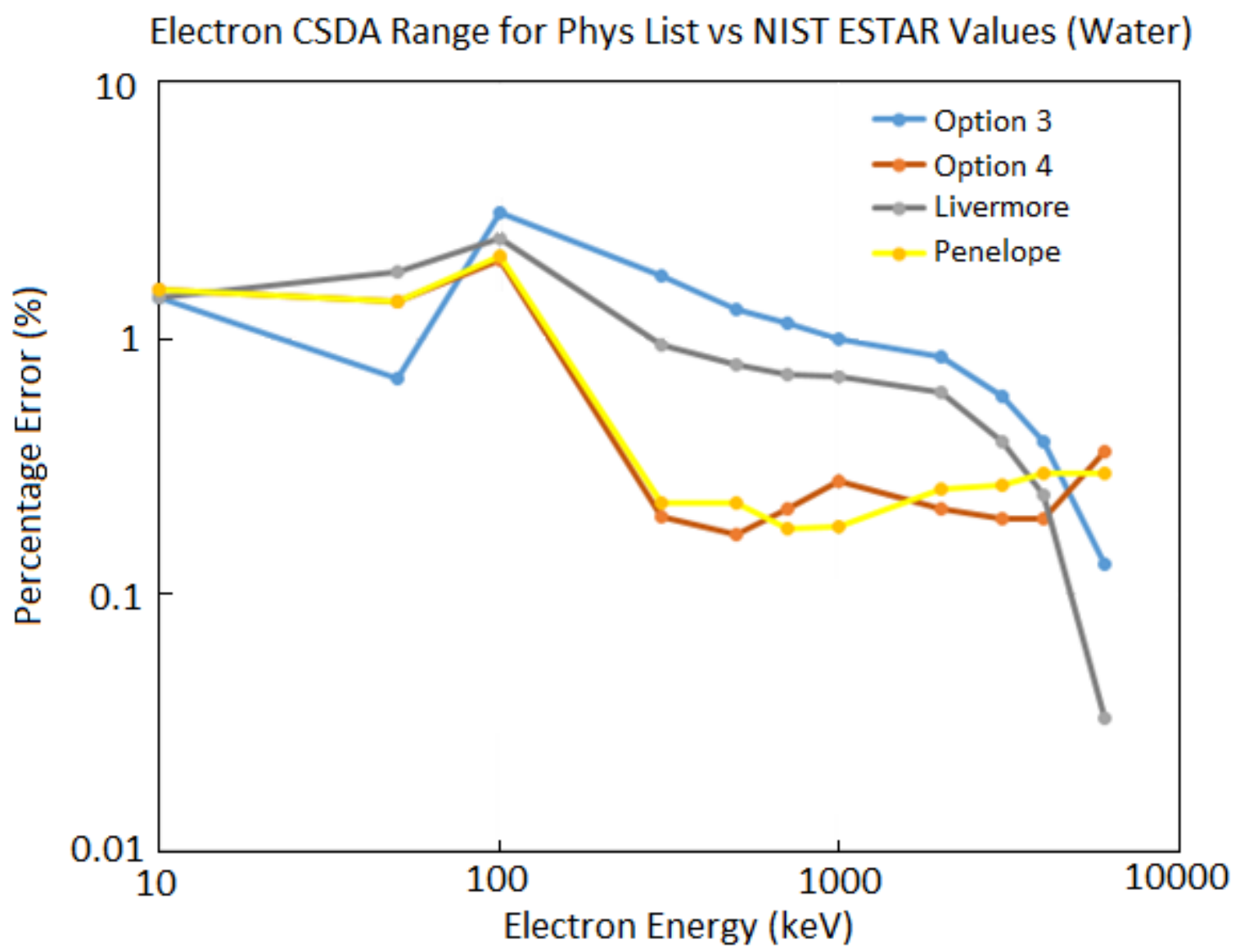

Figure 3.2 The performance for CSDA range and by extension stopping power was performed by firing electrons into an effectively infinite volume of water.

From the overall performances the simulation code received suggested that there were no obvious errors in the coding. The physics list benchmarking showed that Penelope (empenelope) and option 4 (emStandardOption4) were the consistently the most accurate for the energy range of interest. However, since option 4 performed more accurately for the lower energy $\mathrm{x}$-rays it was chosen to be used for the rest of the simulations in the project.

\subsection{Simulation Validation Against Publicized Simulation}

The comparison between the geant4 simulations performed and the EGS4 simulation described in Jaffray's paper was performed using 5 million initial primary $x$-rays per data point shown in the graphs below. The simulations were separated into ten parts to assess the variance and standard deviation for 
each data point. As a result, the error bars in figures 3.3 to 3.7 are smaller than the data points. Errors for Jaffray's data were not visible in many of the figures presented in the papers but due to the low resolution of the images there was some uncertainty in transcribing the data into values. The error bars shown in the graphs represent the widths of the data points plus the widths of the borderlines. While there was error in the horizontal direction these errors were not recorded for the figures since the energy values for the two simulations were chosen to match.

Figure 3.3 shows a comparison between the scatter fraction of the X-Rays passing through the scoring area for mono-energetic $x$-rays ranging in energy from $200 \mathrm{keV}$ to $5 \mathrm{MeV}$. Comparing the simulation results to Jaffray's data the two sets agree within the error of the data extraction. Figure 3.3 shows in a closer inspection that the percentage error between the mean values has a maximum of $1 \%$.

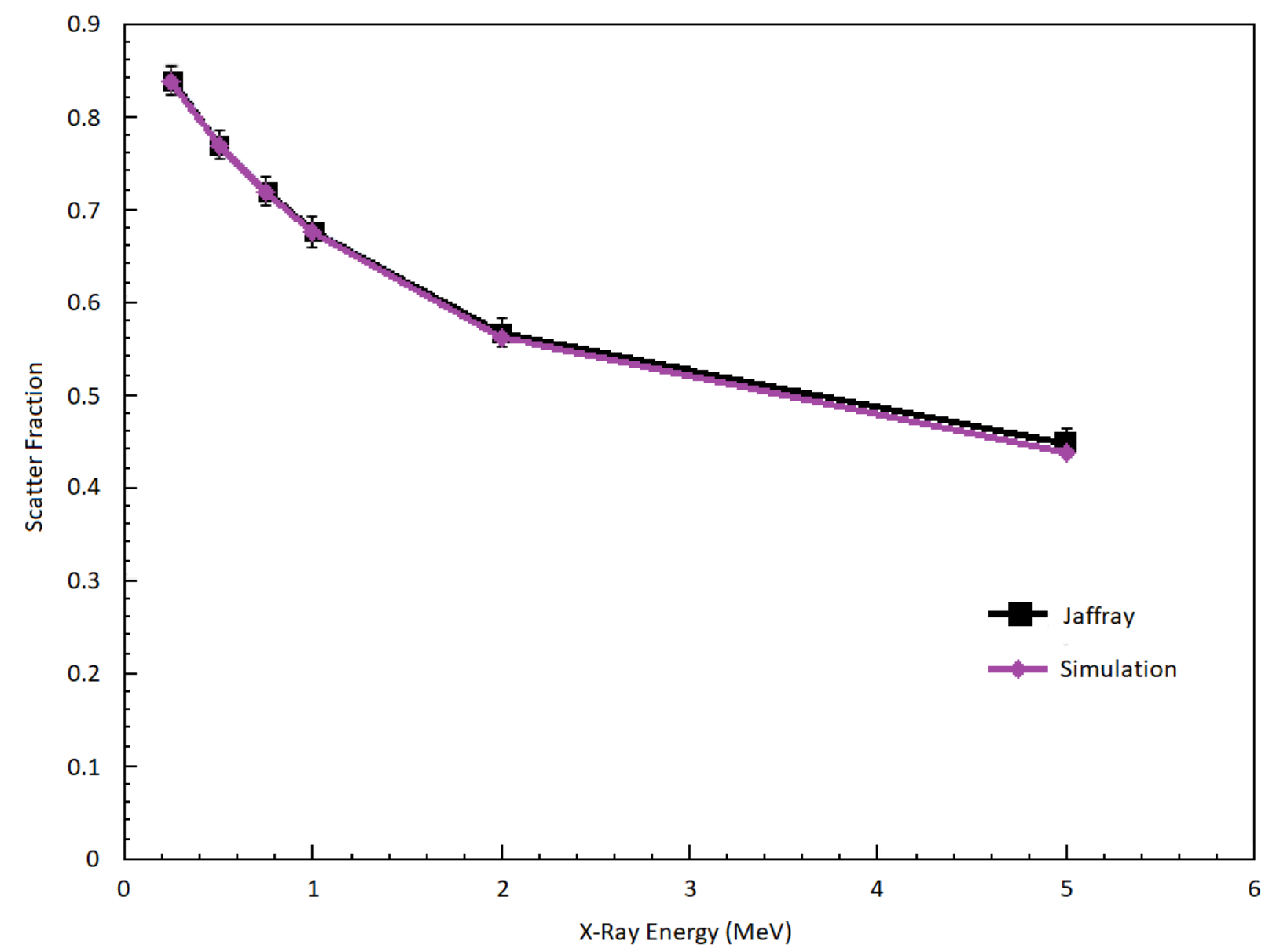

Figure 3.3 The comparison between the two simulations were done with an air gap of 0 and monoenergetic $x$-ray sources. The error bars in Jaffray's data are from the size of the data points and the borders in the original plot. 


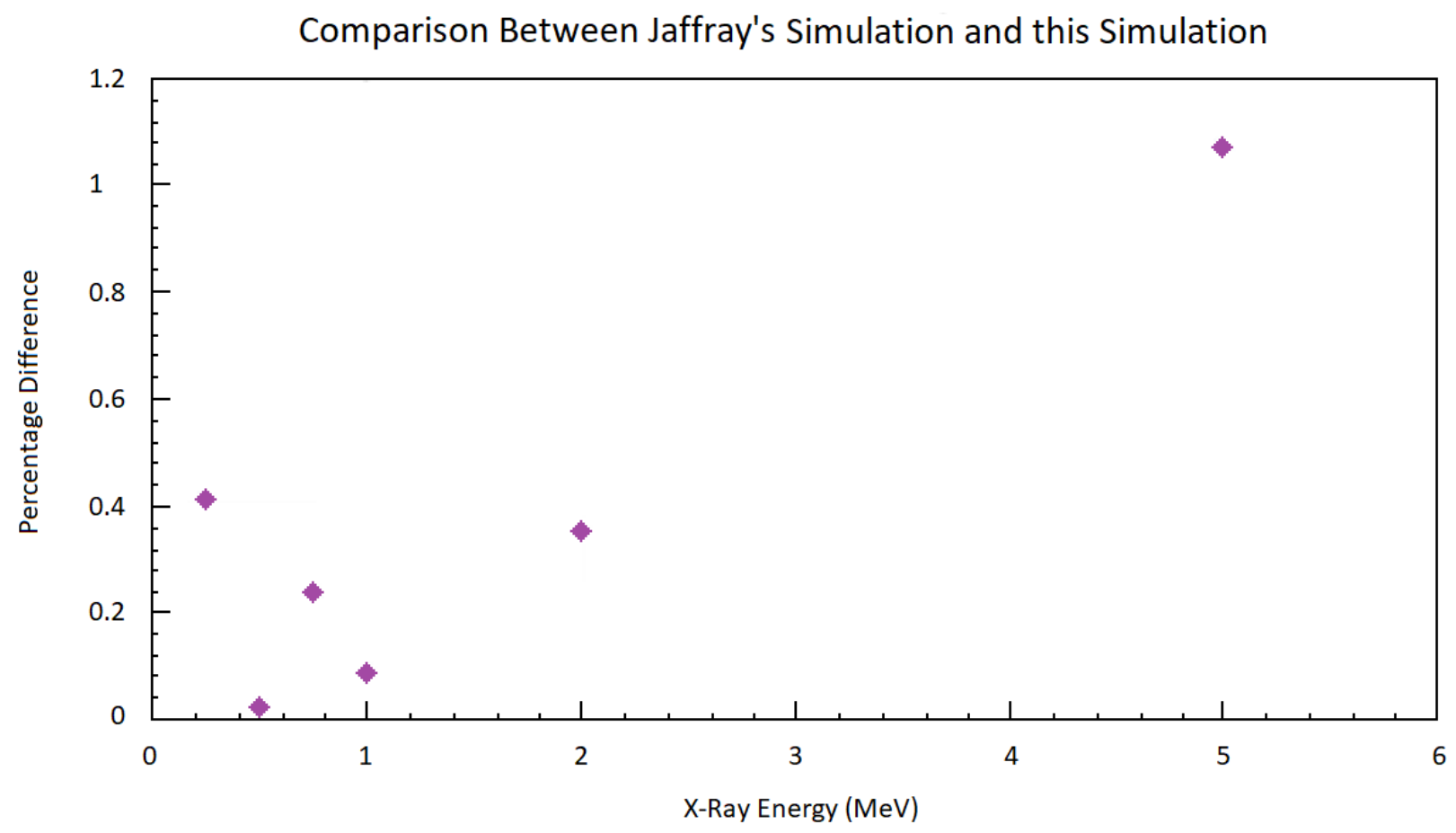

Figure 3.4 The percentage differences for the scatter fraction measurements shown in figure 3.3.

The Primary and scatter fluences were simulated next. Some confusion occurred here since the scatter fraction match is obtained between the two methods yet there is a visible difference between the two scatter fluences. This difference may be reduced when the ratio between the scatter fluence and the total fluence is considered instead of just the scatter fluence. Overall the scatter fluences and the primary fluences lie within the error of the data extraction from Jaffray's paper with the exception of the $2 \mathrm{MeV}$ and $5 \mathrm{MeV} \mathrm{x}$-rays and their scatter fluence. 


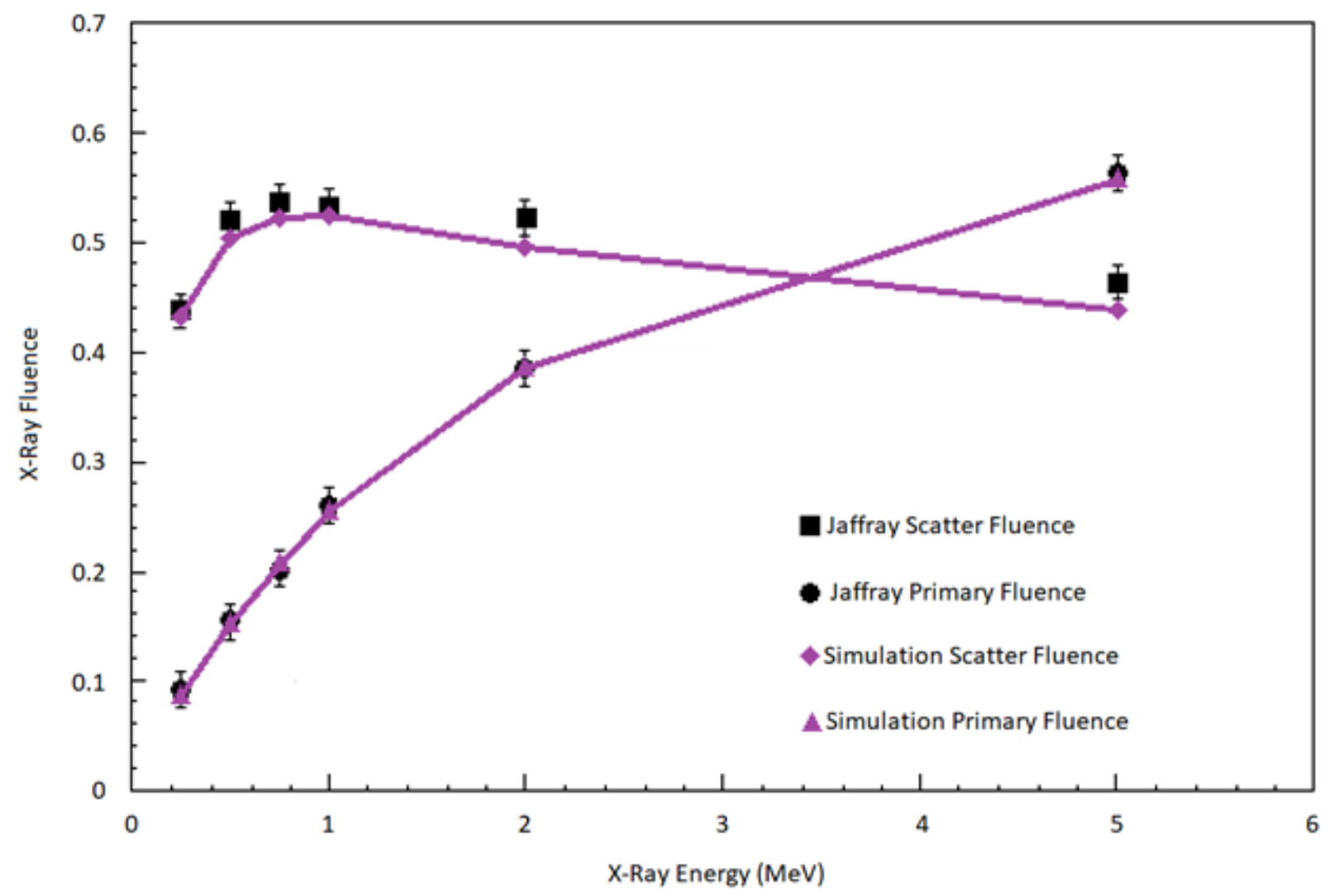

Figure 3.5 X-ray fluence simulations. Primary fluence was calculated by the amount of energy deposited in the phantom. If there was no energy deposited in the phantom, then the $x$-ray interaction with scoring area was primary. The phantom thickness was $17 \mathrm{~cm}$ and the air gap was $0 \mathrm{~cm}$.

From a comparison between the energy spectra generated for $500 \mathrm{keV} x$-rays through the scoring volume and figure 5a from Jaffray's paper similar spectra were obtained. The spectra were normalized to the highest point and the energy scales were matched. The two energy spectra appear to be similar in shape and intensities. 


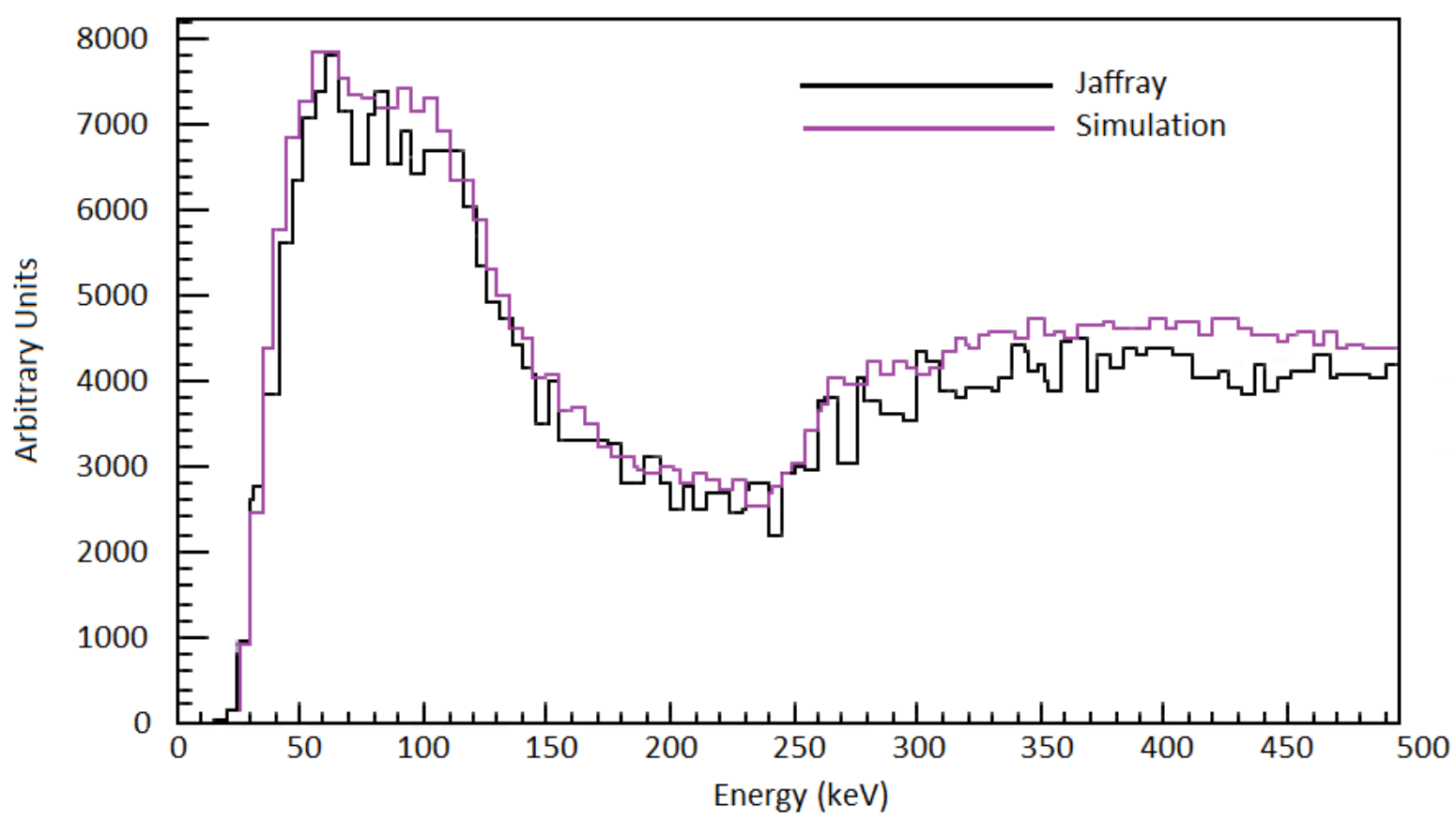

Figure 3.6 A comparison between the energy spectra of a monoenergetic beam of $x$-rays after interaction with $17 \mathrm{~cm}$ thick phantom. The energy was calculated after they passed the scoring volume at an air gap of $0 \mathrm{~cm}$. This is an overlay of this simulations plot and Jaffray's plot. Both the $\mathrm{x}$-axis and the highest point in the peaks were matched.

Finally, the simulation data for figure $8 a$ to $8 c$ in Jaffray's paper were compared to the Geant4 simulation performance. These simulations involved either the phantom thickness, air gap or the beam field size to be varied while the other parameters were held constant. While constant the field size was fixed at $30 \mathrm{~cm}$ by $30 \mathrm{~cm}$, the phantom thickness was fixed at $17 \mathrm{~cm}$ and the air gap was fixed at $30 \mathrm{~cm}$. All simulations were performed with the poly-energetic spectrum shown in figure 2.6. For all three sets of simulations the results were within error of Jaffray's data.

From the results of this simulation comparison it can be said that the Geant4 simulation package being used is valid for the type of simulation outlined in Jaffray's paper. Since the simulations performed for the experimental validation are similar, this simulation validation was sufficient for the simulations performed during the experimental comparison for the prototype MV x-ray image 
Figure 3.7

The rest of the comparisons made with Jaffrays simulations. Unless listed on the X-Axis as varying the air gap was $30 \mathrm{~cm}$, the phantom thickness was $17 \mathrm{~cm}$ and the field size was $30 \mathrm{~cm}$ by $30 \mathrm{~cm}$. A $6 \mathrm{MV}$ poly energetic spectrum was used in for all the measurements. The spectrum can be seen in figure 2.5 .
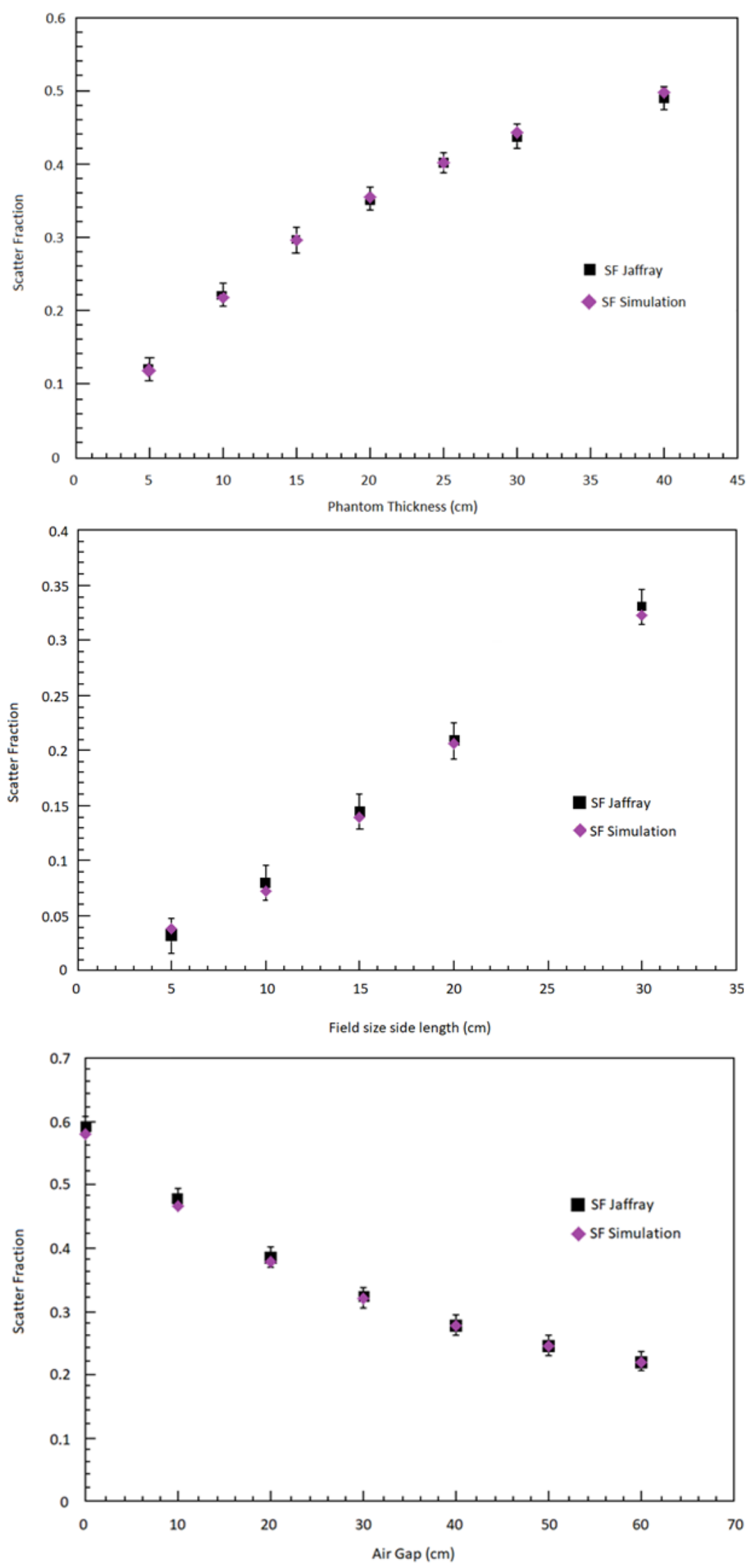


\subsection{Simulation Validation Against Experimental Results}

Experimental verification of the simulation was performed to validate the simulation for the specific application it was designed for. Validation was performed by following the parameters of the experiment as closely as possible given the known details. Both a spatial resolution comparison and a total signal response vs field size comparison was made.

\subsubsection{Spatial Resolution}

MTF match was performed using two sets of simulations with each set containing 36 separate simulations of two-million events each. Each of the 36 simulations were used to scan from one pixel to the next and used in generating an average LSF shown in figure 3.8. Overall the shape of the LSF is similar to the one found in the experiment. For each simulation three iterations of the simulation were performed to determine the uncertainty in each data point obtained using the mean and standard deviation of the three simulations. While the errors appear very small on the edges of the LSF due to the steep slope they are more noticeable near the peak of the distribution. The MTF was convolved with comb function and sinc function. To compare the two functions, the LSF data for the experiment was taken and then re-analyzed using the same method as for the simulation instead of comparing the MTF function directly. This was done since the fitting functions to perform the MTF for the experiment were not clearly indicated, only that the fit was done using three polynomial functions. By using the same method to create both of the MTFs in figure 3.9 less ambiguity is created between the simulated measurement and the experimental measurement. Considering that both the experiment and the simulation used discrete measurements it is appropriate to approach both MTF calculations with a discrete Fourier transform. The Fourier transform was done using the library FTW3 for ROOT analysis software. Similarly, to the LSF measurements the uncertainties were determined using three separate simulations. Uncertainties were determined for both the spatial frequency and the $\operatorname{MTF}(x)$ value.

A comparison between the two LSFs reveals that the LSF for the experiment is narrower than the one for the simulation. When examined the FWHM for the experiment was about $1.68 \mathrm{~mm}$ while the simulation had a FWHM of $1.90 \mathrm{~mm}$ which is 1.13 times larger. The discrepancy between the two values is unknown considering that the two LSF functions were measured in similar ways. Though a possible 
difference may be from scatter in the air since the pencil beam in the simulations were set at twice the distance from the detector than the steel plates. This is a fundamental difference between the simulation and the experiment that was not explored due to time's sake. It is likely that the extra distance in air broadened the pencil beam slightly though it is not known if it would account for a $13 \%$ difference in the FWHM in the LSF. The MTF functions resemble each other from 0 frequency up to around $0.4 \mathrm{lp} / \mathrm{mm}$ afterward the measurements diverge. It is likely that much of the divergence is due to the discrepancy in the widths of the functions since the ratio between the values for the MTF values of 0 resembled the ratios in the FWHM with MTF 0 occurring at around 0.8 for the experiment and 0.7 for the simulation.

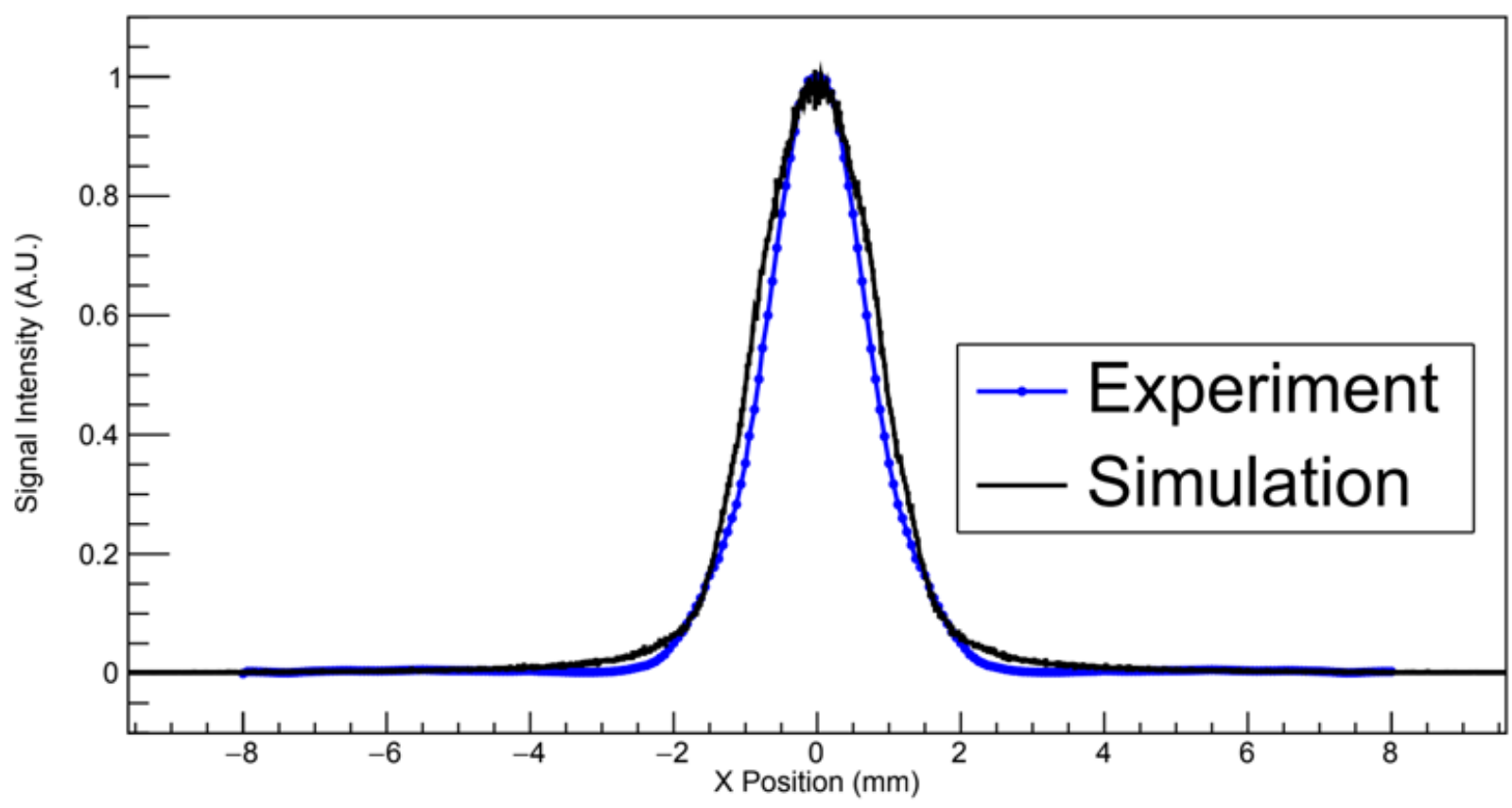

Figure 3.8 A comparison between the simulations and experiments line spread functions along the $x$ axis. The $6 \mathrm{MV}$ polyenergetic spectrum seen in figure 2.6 was used for all simulations of the detector prototype. 


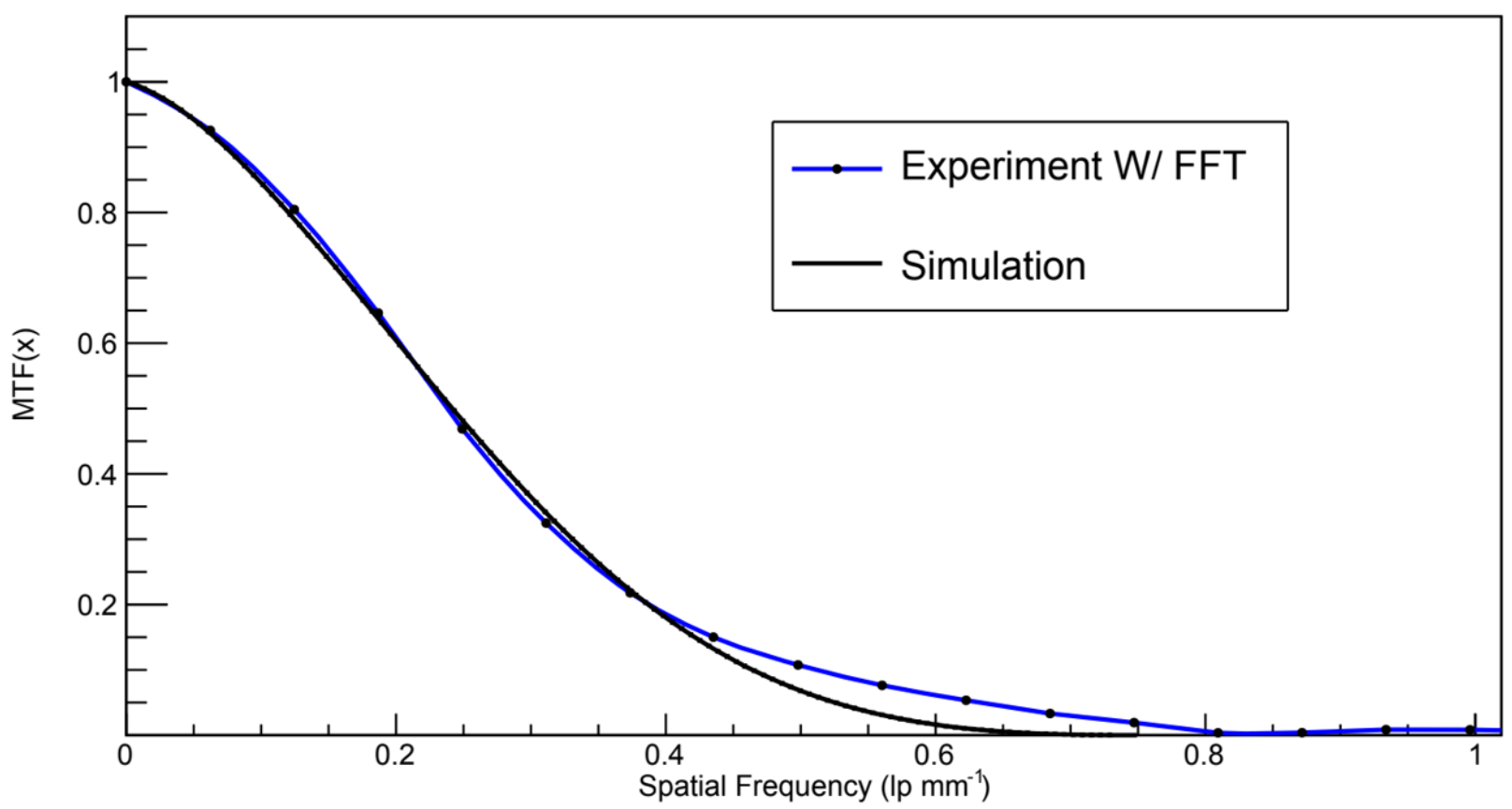

Figure 3.9 The comparison between the modulation transfer functions (MTFs) for experiment and simulation. Both MTF were generated using the same fast Fourier transform code for consistency.

\subsubsection{Total Signal Versus Field Size Comparison}

The total signal was measured for fields sizes from $3.3 \mathrm{~mm}^{2}$ (the area of a single pixel) to a field size of around $600 \mathrm{~cm}^{2}$. Each simulation used a total of 2 million events and were repeated five times to determine the uncertainty in the simulation. Figure 3.10 shows the comparison between the simulated results and experimental measurements. The error bars for the experiment were of similar size to the data points. The data points in the simulation data were hidden and only the error bars are shown thus the thickness of the lines shown in the plot are the uncertainties of the simulations. Three air gaps: $5 \mathrm{~cm}$, $15 \mathrm{~cm}$, and $25 \mathrm{~cm}$, were chosen to be compared to avoid the chance that one set of measurements is a reasonable match despite the simulation being conducted poorly. The maximum percentage differences were measured for each comparison resulting in at most a $2.7 \%+/-0.5 \%$ difference between $5 \mathrm{~cm}$ air gap, $2.6 \%+/-0.5 \%$ difference between $15 \mathrm{~cm}$, and less than $1 \%$ between $25 \mathrm{~cm}$ air gap measurements. The percentage error was determined from the mean and standard deviation from five iterations of the same 
simulation for the largest percentage difference between the experiment and simulation data. It is possible that the discrepancies are due to small errors in the experiment considering that the data points form a rough curve in comparison to the simulation data. The simulation data appears to extrapolate back the same total signal for a field size of $0 \mathrm{~cm}$ by $0 \mathrm{~cm}$, this is supported when examining the raw data. The maximum percentage difference between the total signals for the smallest field size of $3.3 \mathrm{~mm}^{2}$ was $0.3 \%$ supporting the idea that the SPRs would have had a similar comparison if examined directly.

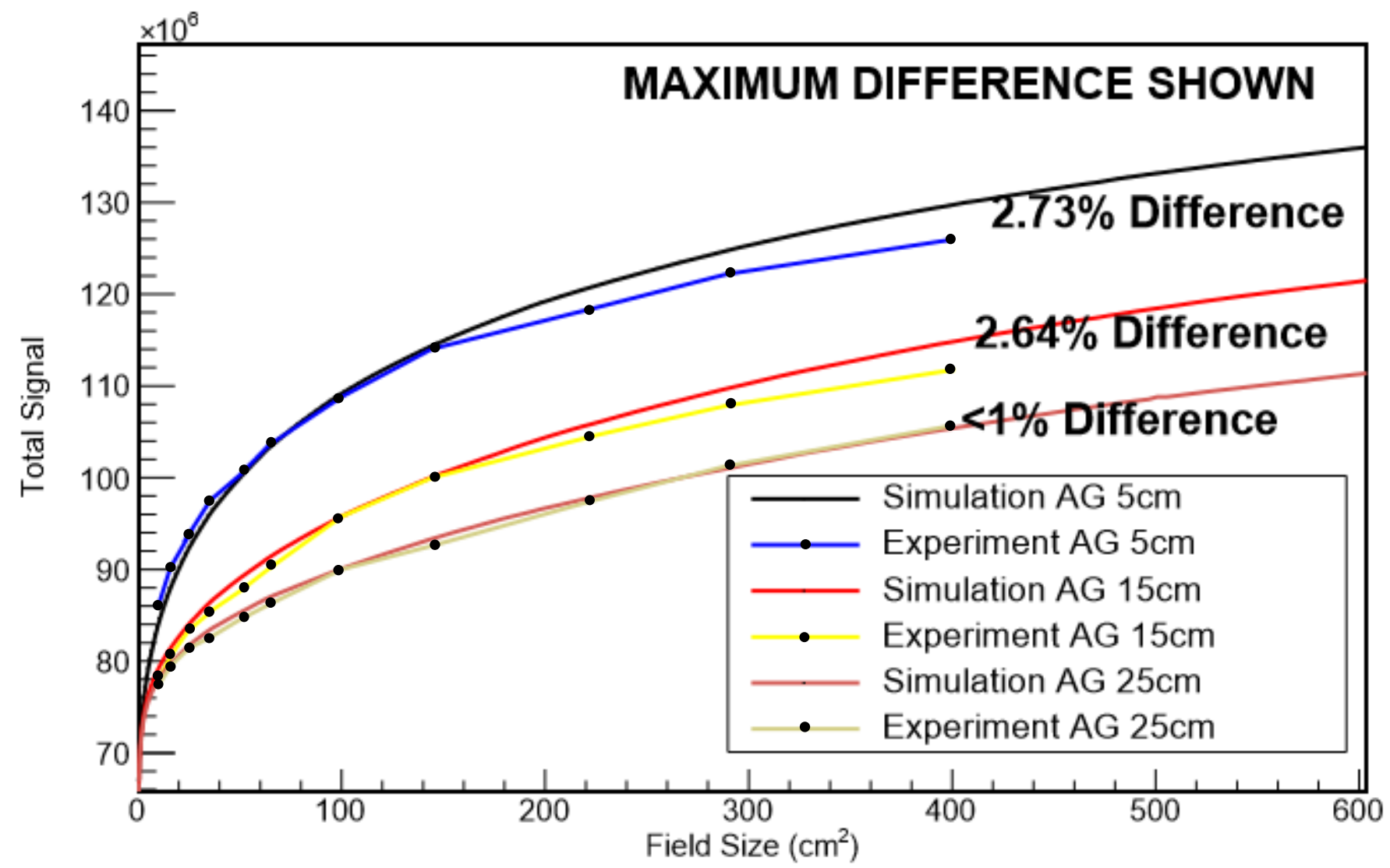

Figure 3.10 A $30 \mathrm{~cm}$ thick phantom of solid water was used centered at a distance of $100 \mathrm{~cm}$ from the beam source. 


\subsection{Optimization}

The primary goals of the optimization were to improve the spatial resolution while keeping the design feasible to built by using commercially available thicknesses of scintillating fibers and lead foils, the glue thicknesses were kept constant at $85 \mathrm{um}$. The design in mind was an array of square fibers in a square array that could be built in a similar fashion to the original detector prototype (see figure 2.15 for more details).

Optimization was done by changing to smaller fibers and then thinner lead foil to minimize the pixel pitch, maximize the spatial resolution and minimize the weight of the detector. Due to time restraints only two types of fibers sizes $1 \mathrm{~mm}$ and $0.5 \mathrm{~mm}$ and two foil thicknesses $0.5 \mathrm{~mm}$ and $0.25 \mathrm{~mm}$ were examined. The first changes made were the fibers and the fiber array which changed from round to square while simultaneously the array was changed from hexagonal to square. From figure 3.11 the initial change from a hexagonal array of round fibers to a square array of square fibers lowered the spatial resolution in the $x$ direction overall from $0.242 \mathrm{lp} \mathrm{mm}^{-1}$ to $0.2166 \mathrm{lp} \mathrm{mm}^{-1}$ for a MTF of 0.5 . This is likely due to the larger pixel size. The square fibers used were $1 \mathrm{~mm}$ wide and the lead was $0.5 \mathrm{~mm}$ thick, with the additional $0.085 \mathrm{~mm}$ of glue, the pixel pitch when from $1.35 \mathrm{~mm}$ for the original design to $1.585 \mathrm{~mm}$ for the new design. The new design was slightly heavier, for a $40 \mathrm{~cm}$ by $40 \mathrm{~cm}$ EPID, the imager weighed 20.7 $\mathrm{kg}$ as opposed to $19.9 \mathrm{~kg}$ for the original design. Changing the pixel size to a width of $0.5 \mathrm{~mm}$ resulted in an improvement over the original detector with $0.319 \mathrm{Ip} \mathrm{mm}^{-1}$ at MTF 0.5 ; however, this detector will have comparably more mass at $26.6 \mathrm{~kg}$. Decreasing the pixel size further to $0.25 \mathrm{~mm}$, the smallest available fiber width from Saint Gobain's, may improve the resolution further but due to time restrictions this was not explored. 


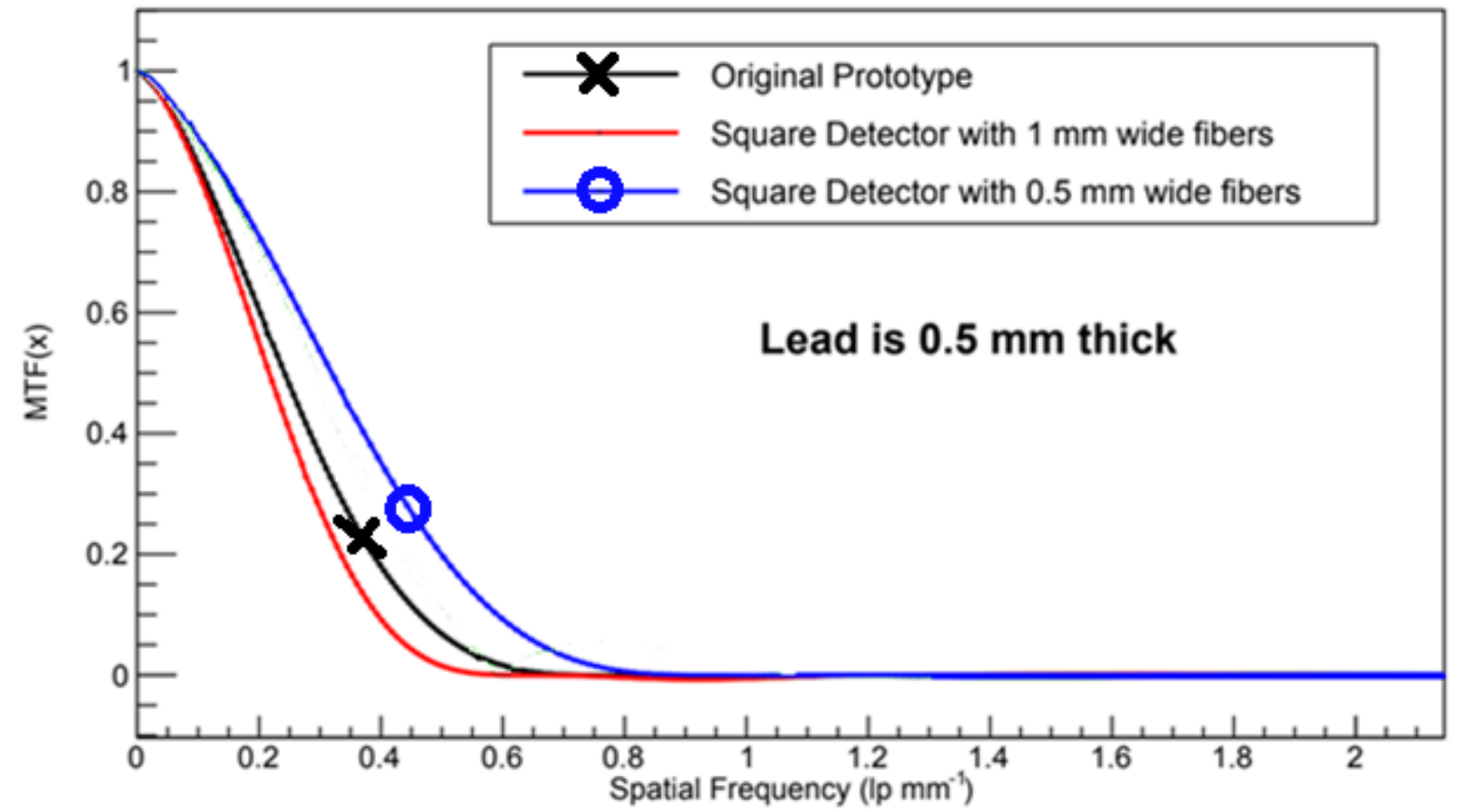

Figure 3.11 A comparison of spatial resolutions for a range of square fiber thicknesses.

Using the most optimized fiber size, $0.5 \mathrm{~mm}$, detector design optimization was explored for a range of lead thicknesses from $0.5 \mathrm{~mm}$ to $0.25 \mathrm{~mm}$. Figure 3.12 shows that further improvement was observed as the lead foil was reduced in thickness, from $0.5 \mathrm{~mm}$ to $0.25 \mathrm{~mm}$, a spatial resolution of 0.359 Ip $\mathrm{mm}^{-1}$ was observed at MTF 0.5. In addition, the mass of this detector design is similar to that of the original design at $20 \mathrm{~kg}$. Reducing the lead thickness further may improve the spatial resolution however due to time restrictions this was not explored. 


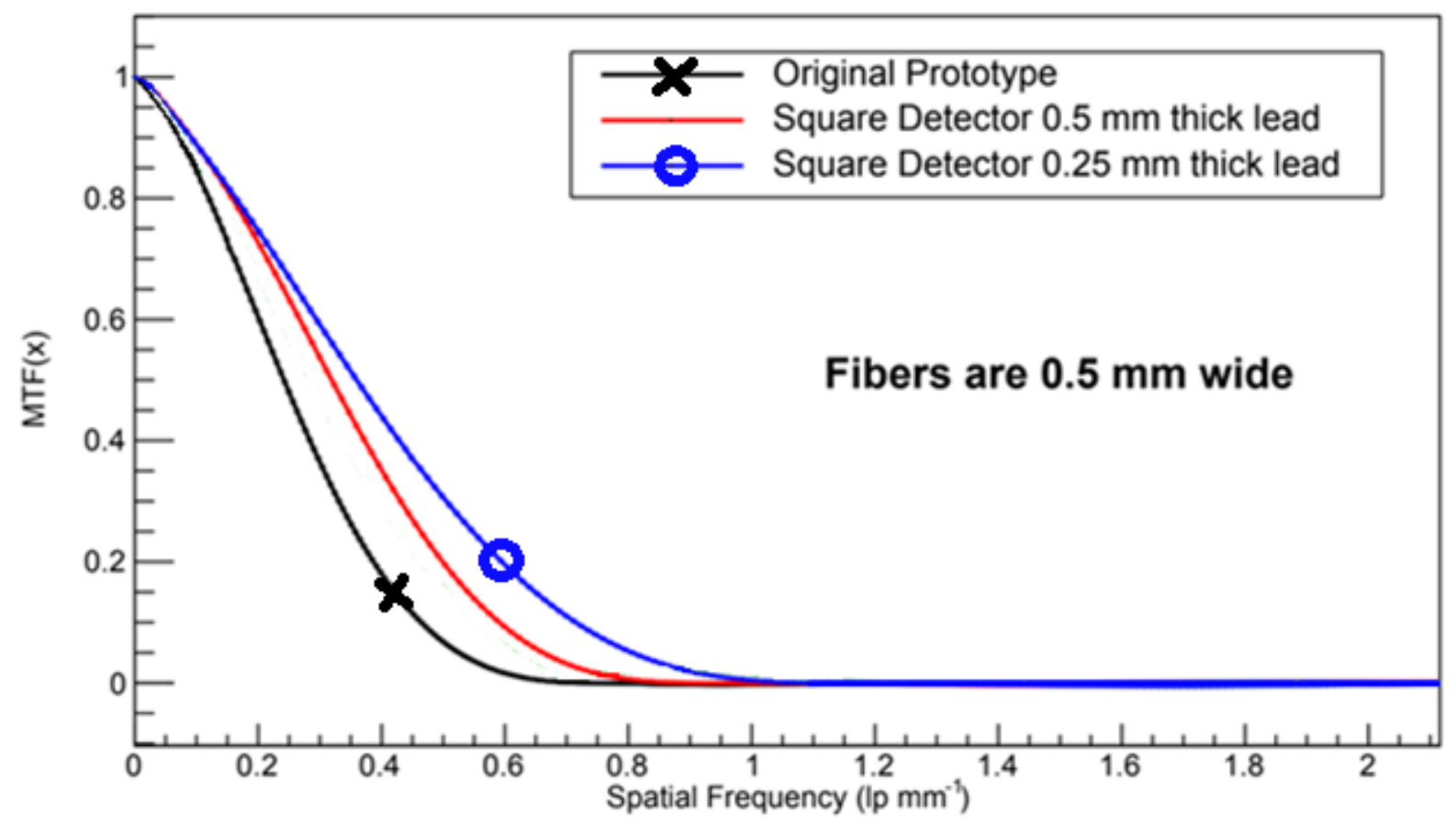

Figure 3.12 A comparison of spatial resolutions for a range of lead foils thickness for the most optimum choice of fiber size. 


\section{Chapter 4}

\section{Discussion}

A slightly thinner foils may increase the detection efficiency further however at some point the more pertinent issue that will arise is the optical epoxy. As the lead foil gets thinner, the thickness of the glue itself becomes significant. Since the glue is optically transparent to a portion of the scintillation light from polystyrene the light leakage between fibers will possibly become significant, degrading the spatial resolution. It is likely that the optimal thickness of foil with optical epoxy is different than with normal epoxy. Whether or not the difference is significant is not known. Thinner foil relative to the size of the pixels has the benefit of reducing the detectors weight which, for the design of the current prototype, is around $20 \mathrm{~kg}$ at the moment. A significant weight may require the LINAC to be rebalanced. In terms of weight requirements, the lightest detector would use relatively large scintillating fibers maximizing the fill factor. The size of the fibers has a similar trade off as the lead, smaller fibers decrease the pixel pitch which may lead to a better spatial resolution. At the moment however the smallest fibers available are $0.25 \mathrm{~mm}$ wide which, unless thinner fibers exist, may be the size limit for the scintillating fibers. Thinner fibers may also have the trade off of light output. Since they are thinner the active area is smaller which gives electrons less area to deposit energy and as a result the light output suffers. For light output concerns, for $1 \mathrm{~mm}$ diameter round fibers single cladded fibers proved to have significantly more light output over the double cladding version. The improved light output is likely due to the larger active area in a single cladded fiber. Since the distance the light must travel is short (1.3 cm on average) the optical photons are likely not travelling far enough to take advantage of the higher light trapping efficiency associated with the double cladded fiber. It is possible that the thinner fibers are more effected by the trapping efficiency since the light will have to go through more internal reflections before reaching the detector.

Optimization can be continued further by changing the thickness of the detector or by finding denser glue. The thickness of the detector would affect how much the generated electrons spread throughout the detector. This would affect the width of the LSF and thus the spatial resolution. A thinner detector may result in a higher spatial resolution but a lower detection efficiency. Ideally the detection efficiency should be above $20 \%$ according to Star-lack et al [17]. Furthermore, the spatial resolution may not significantly depend on the thickness of the detector unless it is very thin; as demonstrated by 
Teymurazyan and Pang [25], where for an already thick detector $(10 \mathrm{~cm})$ increasing the thickness had little effect on the spatial resolution; while a demonstration by Pang and Rowlands [22], showed that decreasing the thickness of a metallic detector from $20 \mathrm{~cm}$ to $0.1 \mathrm{~cm}$ had a noticeable effect on the spatial resolution for a non zero MTF. Logically the prototype detector falls somewhere in between the two cases since while a significant portion of the detector is metallic like the latter case, a significant portion of the detector is also non-metallic similar to the former case.

Changing the glue to something denser would increase the detection efficiency of the detector while not increasing the thickness. Furthermore, it is likely that there is currently a distortion in the spatial resolution along the diagonal directions. Along the glue seams the spatial resolution may be poorer since the stopping power is poorer for the electrons generated in the detector along this direction. This may not be a significant distortion, but it would be worth investigating. By changing to a denser glue this distortion, however significant, would be reduced in magnitude. An alternative would be to somehow solder the lead together or machine the holes for the fibers. Since soldering would likely ruin the fibers and machining thousands of holes for a single detector would significantly increase the cost of the detector, these options are possibly not available. The crimping technique used to make the prototype detector can likely be used to make the square array as well and in large quantities at once thus denser glue is likely the best change in material properties option to improve the detector further. 


\section{Chapter 5}

\section{Conclusion and Future Work}

\subsection{Conclusion}

A reasonable match was obtained between the simulation and the experimental results for spatial resolution and the total signal for various field sizes. The spatial resolutions match up to a spatial frequency of $0.4 \mathrm{Ip} \mathrm{mm}^{-1}$ after which the values deviate up to $\operatorname{MTF}(0)$ value of 0.7 for the simulation and 0.8 for the experiment. The total signal vs field size curves match to within a $3 \%$ difference for an air gap of $5 \mathrm{~cm}$, within $3 \%$ for an air gap of $15 \mathrm{~cm}$ and less than $1 \%$ for an air gap of $25 \mathrm{~cm}$. The discrepancy is thought to be a result of small errors in the experiment due to the roughness of the curves seen in figure 3.10.

After the match was obtained the detector design was changed from a hexagonal array of round fibers to a square array of square fibers. In the limited time remaining in the project the detector design began approaching optimization with the spatial resolution reaching a value of $0.359 \mathrm{lp} \mathrm{mm}^{-1}$ at MTF 0.5 for a square array of $0.5 \mathrm{~mm}$ wide fibers spaced apart by $0.25 \mathrm{~mm}$ thick lead foil. The spatial resolution is likely to be improved further by using a different size of fiber and one of the different thicknesses of foils available provided that the optical epoxy is replaced by something more opaque to prevent light leakage. The thickness of the detector may also have some bearing on the spatial resolution however a thicker detector may not be practical due to possible weight limitations. Having a lower spatial resolution will allow the detector to see finer details in anatomy.

Further optimization can be performed to improve the detection efficiency and thus reduce the amount of dose required for a useable image. The dose requirement is essential for MV CBCT since it requires many useable images to create a reconstruction. Through the use of the new Monte Carlo code the optimization process can be streamlined from both a time perspective and a monetary perspective since the simulations, if set up correctly, are both rigorous and material free. Without human error or unforeseen environmental factors effecting each measurement it is easier to compare one prototype to another. Since the simulation is entirely virtual only a front end investment in a computer with a connection to a computer cluster is required. This allows the materials to be manipulated until the ideal detector is achieved. 
With the new detector MV CBCT will likely be possible with doses closer to those required for KV CBCT. The use of MV CBCT for IGRT will allow for more accurate the tumor targeting and reduced toxicity in healthy tissue. Depending on the counter balance modifications allowed for a LINAC the amount of dose for each image can be significantly reduced up to a theoretical limit of a fiftieth however a limit of a twentieth of the current required dose would be practically feasible. Coupled with using the therapy beam to perform the image the accuracy over KV CBCT will automatically be apparent not only with the use of the correct isocenter but possibly with the direct measurement of the attenuation coefficients required for a treatment plan a more accurate plan can be drafted.

\subsection{Future Work}

In the future the Monte Carlo code written for this project can be used to find the optimal detector design for the application of MV X-ray imaging. The path to the optimal design is probably best executed by paying attention to what sizes of materials are commercially available since special sizes will cost more than standard sizes. The first issue would be the fiber size since this is the most limited in sizes with the smallest fiber size of $0.25 \mathrm{~mm}$ seen in a cursory search. Once the fiber size is optimized it is probably a good idea to think about the glue next. Since the glue that is currently used is optically transparent and much less dense then the lead changing this material will prevent any sort of distortion along the seams. Next optimizing the thickness of the lead between the fibers would be done by following the commercially available thicknesses. Once the spatial resolution is optimized the final parameter to study would be the detector thickness. First a weight limit should be determined and then from the average density of the detector the maximum thickness should be determined. After the prototype is theorized the next step would be to consult a machinist on how difficult it would be to construct this detector. Very thin lead foils will be difficult to work with since lead is very soft and malleable. Once a physical detector is built and installed as intended then tests would have to be performed based on the current standards in imaging. Las Vegas phantom imaging will be required to assess the performance of the new detector against the current detectors. Once the detector is verified and calibrated then clinical trials may begin.

There would likely be two type of prototypes created, one with weight considerations in mind and one that is only volume restricted which would end up being heavier. The weight restricted prototype could be used as a proof of concept detector to show that this design works. While for the heavier prototype design, it may be useful to collaborate with a LINAC manufacturer such as Varian or Elekta since this would make it possible to address the counter balance problems. This would make the new detector 
not immediately available but it would allow the most optimized design to be used. Using the most optimized design is best since it allows for the dose for a MV CBCT to be properly minimized. 


\section{References}

[1] Canadian Cancer Statistics: A 2018 special report. Government of Canada. 2018.

http://www.cancer.ca/ /media/cancer.ca/CW/cancer\%20information/cancer\%20101/

Canadian\%20cancer\%20statistics/Canadian-Cancer-Statistics-2018-EN.pdf?la=en.

[2] External Radiation Therapy. Canadian Cancer Society. 2019.

http://www.cancer.ca/en/cancer-information/diagnosis-and-treatment/ radiation-therapy/external-radiation-therapy/?region=on.

[3] Suntharalingam N, Podgorsak E B, Hendry J H. Basic Radiobiology. IAEA. http://www-naweb.iaea.org/NAHU/DMRP/documents/Chapter14.pdf.

[4] Gupta T. Narayan C A. Image-guided radiation therapy: Physician's perspectives. PMC. 2012. Doi: 10.4103/0971-6203.103602.

[5] Chen J, Morin O, Aubin M, Bucci M K, Chuang CF, Pouliot J. Dose-guided radiation therapy with megavoltage cone-beam CT. The British Journal of Radiology. 2006. DOI: 10.1259/bjr/60612178.

[6] Verellen D, De Ridder M, Tournel K, Duchateau M, Reynders T, Gevaert T, Linthout N, Storme G. An overview of volumetric imaging technologies and their quality assurance for IGRT. Acta Oncologica. 2008. DOI: 10.1080/02841860802244182.

[7] Teymurazyan A. Rowlands J A, Pang G. Monte Carlo simulation of a quantum noise limited Cerenkov detector based on air-spaced light guiding taper for megavoltage x-ray imaging. AAPM. 2014. DOI: $10.118 / 1.4867867$.

[8] Hu Y-H, Rottman J, Fueglistaller R, Myronakis M, Wang A, Huber P, Shedlock D, Morf D, Baturin P, Star-Lack J, Berbeco R. Leveraging multi-layer imager detector design to improve low-dose 
performance for megavoltage cone-beam computed tomography. IPEM. 2018.

DOI: https://doi.org/10.1088/1361-6560/aaa160.

[9] Alaei P, Spezi E. Imaging dose from cone beam computed tomography in radiation therapy.

Physica Medica. 2015. DOI: https://dx.doi.org/10.016/j.ejmp.2015.06.003.

[10] Sillanpaa J, Chang J, Mageras G, Yorke E, De Arruda F, Rosenzweig K E, Munro P, Seppi E, Pavkovich J, Amols H. Low-dose megavoltage cone-beam computed tomography for lung tumors using a high-efficiency image receptor. AAPM. 2006. DOI: 10.1118/1.2222075.

[11] Podgorsak E B. Radiation Physics for Medical Physicists: $2^{\text {nd }}$ edition. Springer. 2010.

ISBN: 978-3-642-00874-0.

[12] Webb A. Introduction to Biomedical Imaging. The Institute of Electrical and Electronics Engineers. 2003. ISBN 0-471-23766-3

[13] Herman M G, Balter J M, Jaffray D A, McGee K P, Munro P, Shalev S, Herk, M V, Wong J W. Clinical use of electronic portal imaging: Report of AAPM Radiation Therapy Committee Task Group 58. AAPM. 2001. DOI: 10.1118/1.1368128.

[14] Antonuk, Larry E. Electronic portal imaging devices:

a review and historical perspective of contemporary technologies and research. Institute of Physics Publishing. 2002. DOI: 10.1088/0031-9155/47/6/201.

[15] Knoll GF. Radiation Detection and Measurement: $3^{\text {rd }}$ ed. John Wiley and Sons Inc. 2000. ISBN: 0471073385.

[16] Prince J L. Links J M. Medical Imaging: Signals and Systems: $2^{\text {nd }}$ Ed. Pearson. 2015. ISBN: 0-13-214518-9. 
[17] Star-Lack J, et al. A piecewise-focused high DQE detector for MV imaging. AAPM. 2015.

DOI: $10.1118 / 1.4927786$.

[18] Rawat T. Digital Signal Processing. Oxford University Press. 2015. ISBN: 978-0-19-808193-7.

[19] Pang G, Rowlands J A. Development of high quantum efficiency flat panel detectors for portal imaging: Intrinsic spatial resolution. AAPM. 2002. DOI: 10.1118/1.1507779.

[20] Aykac M, Uribe J, Baghaei H, Li H, Wang Y, Liu Y, Xing T and Wong W. Septa Design Study for Volumetric Imagig in Positron Emission Tomography. IEEE. 2002. DOI: 10.119/TNS.2002.803812.

[21] Lavine L. Phosphor-plate radiography systems. Dental Economics. 2004.

https://www.dentaleconomics.com/articles/print/volume-94/issue-5/columns/ the-digital-dentist/phosphor-plate-radiography-systems.html.

[22] Pang G, Rowlands J A. Development of high quantum efficiency, flat panel, thick detectors for megavoltage x-ray imaging: A novel direct-conversion design and its feasibility. AAPM. 2004. DOI: $10.1118 / 1.1803771$.

[23] Mei X, Pang G. Development of high quantum efficiency, flat panel, thick detectors for megavoltage x-ray imaging: An experimental study of a single-pixel prototype. AAPM 2005.

DOI: 10.1118/1.2065487.

[24] Mei X, Rowlands J A, Pang G. Electronic portal imaging based on Cerenkov radiation: A new approach and its feasibility. AAPM. 2006. DOI: 10.1118/1.2362875.

[25] Teymurazyan A, Pang G. Megavoltage X-Ray Imaging Based on Cerenkov Effect: A New Application of optical Fibres to Radiation Therapy. International Journal of Optics. 2012.

DOI: $10.115 / 2012 / 724024$. 
[26] Teymurazyan A, Rowlands J A, Pang G. Monte Carlo simulation of a quantum noise limited Cerenkov detector based on air-spaced light guiding taper for megavoltage $\mathrm{x}$-ray imaging. AAPM. 2014. DOI: 10.1118/1.4867867.

[27] Allison J, Amako K, Apostolakis J, Arce P, Asai M, Aso T, Bagli E, Bagulya A, Banerjee S, Barrand G, Beck BR, Bogdanov AG, Brandt D, Brown JMC, Burkhardt H, Canal Ph, Cano-Ott D. et al. Nuclear Instruments and Methods in Physics Research Section A: Accelerators, Spectrometers, Detectors and Asscoiated Equipment. Elsevier. 2016. Vol 835 pages 186- 225. DOI: https://doi.org/10.1016/j.nima.2016.06.125.

[28] Sechopoulos I, Ali ESM, Badal A, Badano A, Boone, JM, Kypriano IS, Mainegra-Hing E, McNitt-Gray MF, McMillan KL, Rogers DWO, Samei E, Turner AC. Monte Carlo Reference Data Sets for Imaging Research: Report of AAPM Task Group 195. AAPM. 2015. DOI: http://dx.doi.org/10.1118/1.4928676.

[29] Tian Y, Pang G. Experimental study of a single-pixel prototype anti-scatter detector for megavoltage x-ray imaging. Radiation Physics and Chemistry. 2016.

DOI: 10.1016/j.radphyschem.2015.09.006.

[30] Usta M, Tufan M. Stopping power and range calculations in human tissues by using the HartreeFock_Roothaan wave functions. Radiation Physics and Chemistry. 2017. DOI: 10.1016/j.radphyschem.2017.03.005.

[31] Jaffray D A, Battista J J, Fenster A, Munro P. X-ray scatter in megavoltage transmission radiography: Physical characteristics and influence on image quality. AAPM. 1994. DOI: https://doi.org/10.1118/1.597255. 
[32] Nelson WR, Hirayama H, Rogers DWO. EGS4 User Manual. SLAC. 1985.

Rcwww.kek.jp/research/egs/epub/manuals/append2.html

[33] S. Kubsad, R. Mackie, B. Gehring, D. Misisco, B. Paliwal, M. Mehta, and T. Kinsella. "Monte Carlo and convolution dosimetry for stereotactic radiosurgery". Science direct. 1990.

DOI: https://doi.org/10.1016/0360-3016(90)90029-j.

[34] Liu J. A Novel High Quantum Efficiency MV X-Ray Detector for Image-guided Radiotherapy. Ryerson University. 2018.

[35] Fujita H, Tsai D, Itoh T, Doi K, Morishita J, Ueda K, Ohtsuka A. A Simple Method for Determining the Modulation Transfer Function in Digital Radiography. IEEE Transactions on Medical Imaging. 1992. DOI: $10.1109 / 42.126908$.

[36] Teymurazyan A, Pang G. An inherent anti-scatter detector for megavoltage x-ray imaging. IOP Publishing. 2013. DOI: 10.1088/0031-9155/58/5/1479.

[37] Compound Summary: Tetrafluoroethylene. US National Library of Medicine. https://pubchem.ncbi.nlm.nih.gov/compound/ tetrafluoroethylene\#section=Information-Sources.

[38] Moll M. Radiation damage in scintillating fibers: Preliminary literature study. Cern. 2003. https://mmoll.web.cern.ch/mmoll/publist/talks/ 031015-Radiation\%20Damage\%20in\%20Fibers.pdf. 